\title{
Checklist of the terrestrial isopods of the new world (Crustacea, Isopoda, Oniscidea)
}

\author{
Andreas Leistikow ${ }^{1,2}$ \\ Johann Wolfgang Wägele ${ }^{2}$
}

\begin{abstract}
A check-list of all the American Oniscidea known to the authors and their quotation in literature is presented. The species account comprises notes on species' distribution and a revised synonymy. As far as possible comments on taxonomic problems are given. The species are ascribed to the families which are commonly recognised, despite many of them are paraphyletic constructions. This check-list should support the work of both ecologists and taxonomist when dealing with New World Oniscidea.

KEY WORDS. Isopoda, American Oniscidea, taxonomy, biodiversity, check-list
\end{abstract}

The suborder Oniscidea is one of the most important within the Isopoda with almost half of all known species of Isopoda belonging to it. The members of Oniscidea play an important role in terrestrial ecosystems, especially in the tropics. They are destruents occurring in great numbers and some were able to adapt to man. Therefore, they became anthropophilous and are cosmopolitically distributed like Porcellionides pruinosus (Brandt, 1833) and Cubaris murina Brandt, 1833.

A first attempt to review the distributional patterns of Oniscidea had been made by VANDEL (1945), but until this time the knowledge on the distribution and diversity of terrestrial isopoda has increased considerably in the last decades. Unfortunately, there are no new monographic works on the suborder. At least, there are check-lists on Oniscidea from Oceania (JACKSON 1941) and Africa south of the Sahara (FERRARA \& TAITI 1978). For the Americas, the last review on fresh water and terrestrial isopods was undertaken by VAN NAME $(1936,1940,1942)$. Since then, the number of species described mainly from South America has almost doubled. Hence, it is desirable to give a summary of the species described from the Americas and their quotation in literature in form of a check-list to fill this gap.

In the following list species which were most probably introduced to the New World by human activity are indicated by a double cross (\#). Beside the records from the Americas, the native distribution of those species is given as far as it was reconstructable. Whereever possible, some comments on the families, genera and species are made to focus on outstanding taxonomic problems which have to be solved in future works. All the available synonyms published since 1942 are listed, and the name published most recently was accepted as valid where new reconsideration was not possible.

1) Universität Bielefeld, Fakultät für Biologie, Abteilung für Morphologie und Systematik der Tiere. Morgenbreede 45, D-33615 Bielefeld, Germany.

e-mail: leiste@biologie.uni-bielefeld.de

2) Ruhr-Universität Bochum, Fakultät für Biologie, Lehrstuhl für spezielle Zoologie. Universitätsstraße 150, D-44780 Bochum, Germany. 
The bibliography indicates all the contributions available to the authors which were published since the last supplement of van Name's work on American land and fresh water isopods (VAN NAME 1942). In cases where it was necessary for clarification of taxonomic and nomenclatoric questions, older literature is cited.

\section{SPECIES ACCOUNT}

\section{Ligiidae Brandt \& Ratzeburg, 1831 \\ Ligia Fabricius, 1798}

Ligia baudiniana Milne-Edwards, 1840

Literature: Miers (1877); ArCANGeli (1930); CreAser (1936); VAN NAME (1936); Andersson (1960); Mulaik (1960); Schultz (1974b); Schultz (1984c); MUCHMORE (1993); LEISTIKOW (1997a)

Distribution: Florida to Brazil; West Indies; Ecuador, Subida Alta, Puna Island; Colombia, Buenoventura; Mexico, Baja California; Costa Rica, Corcovado; Galapagos (?)

Ligia cajennensis Koch, 1847

Literature: VAN NAME (1936)

Distribution: Cayenne

Ligia cinerascens Budde-Lund, 1885

Literature: VAN NAME (1936)

Distribution: Chile, doubtful record

Ligia exotica Roux, 1828

Synonymy: Ligia olfersi Brandt, 1833

Literature: VAN NAME (1936); ANDERSSON (1960); MULAIK (1960); RECA (1972); SCHUltz (1974b); SCHMAlFuss \& FERRARA (1978); SCHULTZ \& Joinson (1984)

Distribution: pantropic, in America from the USA, North Carolina to Argentinia, Buenos Aires

Ligia filicornis Budde-Lund, 1893

Literature: VAN NAME (1936)

Distribution: Venezuela, Puerto Cabellos

Remark: synonym of Ligia exotica Roux, 1828 ?

Ligia hawaiensis Dana, 1853

Literature: VAN NAME (1936)

Distribution: Pacific Islands, Mexico (doubtful record)

Ligia novae-zealandiae Dana, 1852

Literature: VAN NAME (1936); ANDERSSON (1960); STROUHAL (1961);

Distribution: Peru, Chincha Island; Chile, Valparaiso, Tierra del Fuego, Juan Fernandez (ssp. litigrosa Wahrberg, 1922); New Zealand

Ligia occidentalis Dana, 1853

Literature: vAN NAME (1936); BowmAn (1977); GARTHWAITE et al. (1985); GARTHWAITE \& LAWSON (1992)

Distribution: USA, California; Mexico, Baja California

Ligia oceanica (Linné, 1767) \#

Literature: VAN NAME (1936)

Distribution: USA, Massachusetts, Rhode Island

Native distribution: Western Europe, coasts of Atlantic and North Sea 
Ligia pallasii Brandt, 1833

Literature: VAN NAME (1936); H^TCH (1947); GARTHWAITE (1992)

Distribution: USA, Alaska to Central California; Western Canada, Aleutes

Ligia platycephala van Name, 1927

Synonymy: Ligia callani Collinge, 1946

Ligia muscorum Jackson, 1927

Literature: vAN NAME (1936); COLlinge (1946); VANDEL (1952b); SChUltz (1974b)

Distribution: Guyana; Trinidad

Ligia simoni (Dollfus, 1896)

Literature: DollFus (1896c); VAN NAME (1936); SchMALFuSS (1978)

Distribution: Venezuela, Cumbre de Valencia; Colombia, Santa Marta

\section{Ligidium Brandt, 1833}

Ligidium blueridgensis Schultz, 1964

Literature: SCHULTZ (1964a)

Distribution: USA, North Carolina

Ligidium elrodii (Packard, 1873)

Synonymy: Ligidium longicaudatum Stoller, 1902

Ligidium hypnorum (Cuvier, 1792) partim

Literature: VAN NAME (1936); VAN NAME (1940); CAUSEY (1952); Schultz (1970c); JASS \& KlAUSmeier (1990); SNIDER (1991)

Distribution: Canada, Ontario; Northeastern parts of USA

Ligidium floridanum Schultz, 1974

Literature: SCHULTZ (1974b)

Distribution: USA, Florida

Ligidium gracile (Dana, 1856)

Literature: VAN NAME (1936); Hatch (1947); GarThWAite \& LAWSON (1992)

Distribution: USA, Alaska to Central California; Western Canada

Ligidium kofoidi Maloney, 1930

Literature: VAN NAME (1936)

Distribution: USA, California

Ligidium lapetum Mulaik, 1942

Literature: VAN NAME (1942); GARTHWAITE et al. (1985)

Distribution: USA, California

Ligidium latum Jackson, 1923

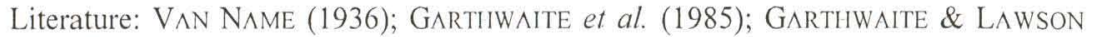
(1992)

Distribution: USA, California

Ligidium mucronatum Mulaik, 1942

Literature: VAN NAME (1942)

Distribution: USA, Louisiana

Stymphalus Budde-Lund, 1885

Stymphalus dilatatus (Perty, 1834)

Literature: V^N NAME (1936)

Distribution: Brazil, Bahia 


\section{Tylidae Milne-Edwards, 1840 \\ Tylos Audouin \& Savigny, 1826}

Tylos chilensis Schultz, 1983

Literature: SCHULTZ (1983a)

Distribution: Chile, Valparaiso

Tylos insularis van Name, 1936

Literature: VAN NAME (1936)

Distribution: Galapagos Islands

Tylos latreillei (Audouin \& Savigny, 1826)

Literature: VAN NAME (1936); VANDEL (1952a); MULAIK (1960); SCHULTZ \& JOHNSON (1984)

Distribution: Mediterranean and Caribbean Seas: coasts of Mexico, Honduras; Venezuela; Puerto Rico; USA, Florida

Tylos marcuzzii Soika, 1954

Literature: SCHUltz(1974b); SchulTz (1984c); SCHULTZ \& JohnSON (1984); GARCÉS (1991)

Distribution: USA, Florida; Bahamas; Belize; Venezuela, Isla Margarita

Tylos niveus Budde-Lund, 1885

Literature: Van Name (1936); Lemos de Castro (1952); VAndel (1952b); Mulaik (1960); Lemos de CASTRO (1971); SChUlTZ (1974b);SChulTZ \& JohnSON (1984); SCHULTZ (1984c); MUCHMORE (1993)

Distribution: USA, Florda, Virgin Islands; Cuba; Caribbean Sea; Venezuela; Brazil, Rio de Janeiro (if introduced ?)

Tylos punctatus Holmes \& Gay, 1909

Literature: VAN NAME (1936); VAN NAME (1940); GARTHWAITE et al. (1985)

Distribution: USA, California; Mexico, Guyamas

Tylos spinulosus Dana, 1853

Literature: VAN NAME (1936); SCHULTZ (1983a)

Distribution: Chile, Tierra del Fuego to Punta Choros

Tylos wegeneri Vandel, 1952

Literature: VANDEL (1952b); SChUlTZ (1983a); GARCÉS(1991)

Distribution: Venezuela, Isla Margarita; Costa Rica; USA, Florida

Trichoniscidae Sars, 1899

Amerigoniscus Vandel, 1950

Amerigoniscus centralis Vandel, 1978

Literature: VANDEL (1978)

Distribution: USA, Oklahoma

Amerigoniscus curvatus Vandel, 1978

Literature: VANDEL (1978)

Distribution: USA, Georgia

Amerigoniscus georgiensis Vandel, 1978

Literature: VANDEL (1978)

Distribution: USA, Georgia 
Amerigoniscus gipsicolus (Vandel, 1965)

Synonymy: Caucasonethes gipsicolus Vandel, 1965

Literature: VANDEL (1965b); VANDEL (1978)

Distribution: USA, New Mexico

Amerigoniscus henroti (Vandel, 1950)

Synonymy: Caucasonethes henroti Vandel, 1965

Literature: VANDEL (1950); VANDEL (1965b); HOLSINGER (1967); VANDEL (1978)

Distribution: USA, Virginia

Amerigoniscus malheurensis Schultz, 1982

Literature: SCHULTZ (1982)

Distribution: USA, Oregon

Amerigoniscus nicholasi (Vandel, 1965)

Synonymy: Caucasonethes nicholasi Vandel, 1965

Caucasonethes paynesi Muchmore, 1970

Literature: VANDEL (1965b); MUCHMORE (1970); VANDEL (1978)

Distribution: USA, Tennessee

Amerigoniscus proximus Vandel, 1978

Literature: VANDEL (1978)

Distribution: USA, Georgia

Amerigoniscus rothi (Vandel, 1953)

Synonymy: Caucasonethes rothi Vandel, 1953

Literature: VANDEL (1978); SCIIULTZ (1981)

Distribution: USA, Oregon

\section{Andronsicus Verhoeff, 1908}

Androniscus dentiger Verhoeff, 1908 \#

Synonymy: Trichoniscus (Androniscus) dentiger Verhoeff, 1908

Literature: VAN NAME (1936); PALmÉn (1951); JASS \& KLAUSMEIER (1990)

Distribution: Canada, Newfoundland, Ontario

Native distribution: Nordwestern Europe

\section{Brackenridgia Ulrich, 1902}

Brackenridgia acostai (Rioja, 1951)

Synonymy: Protrichoniscus acostai Rioja, 1951

Literature: RIOJA (1951b); RIOJA (1955c); MULAIK (1960); SCHULTZ (1984b)

Distribution: Mexico, Chiapas

Brackenridgia bridgesi (van Name, 1942)

Synonymy: Protrichoniscus bridgesi van Name, 1942

Protrichoniscus potosinus Mulaik, 1960

Literature: VAN NAME (1942); RIOJA (1955c); MulAIK (1960); VANDEL (1965b); SCHULTZ (1984b)

Distribution: Mexico, San Luis Potosí, Tamaulipas

Brackenridgia cavernarum Ulrich, 1902

Synonymy: Protrichoniscus cavernarum Vandel, 1965

Literature: VAN NAME (1936); VANDEL (1965b); Schultz (1984b)

Distribution: USA, Texas 
Brackenridgia heroldi (ARCANGELI, 1932)

Synonymy: Protrichoniscus heroldi ArCANGeli, 1932

Literature: VAN NAME (1936); Rioja (1955c); GARTHWAITE et al. (1985); GARTHWAITE (1992)

Distribution: USA, California

Brackenridgia reddelli Vandel, 1965

Synonymy: Protrichoniscus reddelli Vandel, 1965

Literature: VANDEL (1965b)

Distribution: USA, Texas

Brackenridgia villalobosi (Rioja, 1950)

Synonymy: Protrichoniscus villalobosi Rioja, 1950

Literature: Rioja (1950, 1955c); Mulaik (1960); VANDEL (1965b); Schultz (1984b)

Distribution: Mexico, Veracruz

Cylindroniscus ARCANGELI, 1929

Cylindroniscus cavicolus (Mulaik, 1960)

Synonymy: Antroniscus cavicolus Mulaik, 1960

Literature: MULAIK (1960); SCIIULTZ (1970e)

Distribution: Mexico, Nuevo León

Cylindroniscus maya Rioja, 1957

Synonymy: Antroniscus balamensis Mulaik, 1960

Literature: Rioja (1957); Mulaik (1960); SCHUltz (1970e); VANDEL (1981)

Distribution: Mexico, Yucatán

Cylindroniscus seurati ARCANGELI, 1929

Literature: VAN NAME (1936); Schultz (1970e); VANDEl (1973); Schultz (1981); VANDEL (1981)

Distribution: Cuba, Guayabal

Cylindroniscus vallesensis Schultz, 1970

Literature: ScHULTZ (1970e)

Distribution: Mexico, San Luis Potosí

Cylindroniscus yucatanensis (Mulaik, 1960)

Synonymy: Antroniscus yucatanensis Mulaik, 1960

Literature: MulAIK (1960); SCHULTZ (1970e)

Distribution: Mexico, Yucatán

\section{Haplophthalmus Schöbl, 1860}

Haplophthalmus danicus Budde-Lund, 1877 \#

Literature: VAN NAME (1936); VANDEL (1950); PALMÉN (1951); Mulaik (1960);

VAndel (1965b); Lemos de Castro (1971); VANdel (1977); Garthwaite et al. (1985); KEENEY (1990)

Distribution: Canada, Newfoundland; USA, Indiana, California, Kentucky, New Jersey,

New York; Ohio, Tennessee; Mexico; Brazil, São Paulo; St. Helena

Native distribution: Europe

Hyloniscus Verhoeff, 1908

Hyloniscus riparius (Koch, 1838) \#

Literature: Palmén (1951); Schultz (1963a); Jass \& Klausmeier (1990); SNider (1991); JASS \& KLAUSMEIER (1996)

Revta bras. Zool. 16 (1): 1 - 72, 1999 
Distribution: Canada, Newfoundland; USA, Wisconsin, New York, Pennsylvania, Michigan, Wisconsin

Native distribution: Europe

\section{Mexiconiscus SCHULTZ, 1964}

Mexiconiscus laevis (Rioja, 1955)

Synonymy: Cordioniscus laevis Rioja, 1955

Xilitloniscus laevis Bowman, 1965

Mexiconiscus thlamayensis SCHULTZ, 1964

Literature: Rioja (1955a); Scilultz (1964b); BowmAn (1965); Schultz (1965b),

VANDEL (1970); SCHULTZ (1981); SCHULTZ (1994)

Distribution: Mexico, Hildago, San Luis Potosí

\section{Miktoniscus Kesselyak, 1930}

\section{Miktoniscus barrai Vandel, 1965}

Literature: VANDEL (1965b); SChultz (1976); JASS \& KLAUSMEIER (1990)

Distribution: USA, Tennessee, Indiana, Massachusetts, Georgia, North Carolina

Miktoniscus halophilus Blake, 1931

Synonymy: Miktoniscus grayi SCIULTZ, 1962

Literature: VAN NAme (1936); Schultz (1962); Schultz (1975); Schultz (1976); SCHULTZ (1977a)

Distribution: USA southwards to Georgia

Miktoniscus medcofi van Name, 1940

Synonyme: Miktoniscus humus Mulaik, 1960

Miktoniscus linearis (Patience, 1908) partim

Miktoniscus alabamensis Muchmore, 1964

Miktoniscus ohioensis Muchmore, 1964

Trichoniscus veracrucensis Mulaik, 1960

Literature: VAN NAME (1940); VANDEL (1950); LEMOS DE CASTRO (1953); MULAIK (1960); MUChMORL (1963); SCHULTZ (1964a); VANDEL (1965b); PECK (1970); LEMOS de Castro (1971); Schultz (1976); Jass \& Klausmeier (1996)

Distribution: USA, Alabama, Ohio, Louisiana, Florida, also in greenhouses; SO Brazil (introduced?)

Miktoniscus morganensis Schultz, 1976

Literature: SCIIULTZ (1976)

Distribution: USA, Alabama

Miktoniscus oklahomensis Schultz, 1981

Literature: ScHULTZ (1981)

Distribution: USA, Oklahoma

Miktoniscus racovitzai Vandel, 1950

Literature: VANDEL (1950); MUCHMORE (1963); VANDEL (1965b); SCHULTZ (1976); SCHULTZ (1981)

Distribution: USA, Virginia, Oklahoma

Oregoniscus Hatch, 1947

Oregoniscus nearcticus (ARCANGELI, 1932)

Synonymy: Trichoniscus nearcticus ARCANGELI, 1932

Literature: VAN NAME (1936); НАTCH (1947)

Distribution: USA, Oregon 


\section{Trichoniscoides Sars, 1899}

Trichoniscoides sarsi Patience, 1908 \#

Literature: PALMÉN (1951)

Distribution: Canada, Newfoundland

Native distribution: Northwestern Europe

Trichoniscus Brandt, 1833

Trichoniscus demivirgo Blake, 1931

Literature: VAN NAME (1936); VAN NAME (1940); HATCH (1947); CAUSEY (1952)

Distribution: USA, New England, Washington, Arkansas; Canada, Ontario, Nova Scotia

Trichoniscus hoctuni Mulaik, 1960

Literature: MULAIK (1960)

Distribution: Mexico, Yucatán

Trichoniscus orchidicola Mulaik, 1960

Literature: MULAIK (1960)

Distribution: USA, Texas, in supply of mexican orchids

Trichoniscus provisorius Racovitza, 1908 \#

Literature: PALMÉN (1951)

Distribution: Canada, Newfoundland

Native distribution: Europe

Trichoniscus pseudopusillus Arcangeli, 1929

Literature: VAN NAME (1936)

Distribution: Cuba

Trichoniscus pusillus Brandt, 1833 \#

Literature: PALMÉN (1951); VANDEL (1977); VANDEL (1981); KEEENY (1990); JASS \& KLAUSMEIER (1990); SNIDER (1991)

Distribution: Canada, Newfoundland; eastern USA; Cuba; St. Helena

Native distribution: Europe

Trichoniscus pygmaeus Sars, 1899 \#

Literature: VAN NAME (1936); PALMÉN (1951); JASS \& KLAUSMEIER (1990)

Distribution: Canada, Newfoundland; USA, Illinois, New York

Native distribution: Western Europe

Trichoniscus species Hatch, 1947

Similar to Trichoniscus pusillus Sars, 1899

Literature: H^TCII (1947)

Distribution: greenhouse in Oregon

Typhlotricholigoides Rioja, 1952

y phlotricholigoides aquaticus Rioja, 1952

Literature: RioJA (1952, 1955c); VANDEL (1965a); SCIIULTZ (1981); SCHULTZ (1994)

Distribution: Central Mexico

Styloniscidae Vandel, 1952

Clavigeroniscus Arcangeli, 1930

Clavigeroniscus alticolus Vandel, 1972

Literature: VANDEL (1972a)

Distribution: Colombia, Montserrate

Revta bras. Zool. 16 (1): 1 - 72, 1999 
Clavigeroniscus orghidani Vandel, 1981

Literature: VANDEL (1981)

Distribution: Cuba

Clavigeroniscus riqueri Arcangeli, 1930

Literature: ARCANGELI (1930); VAN NAME (1936), VAN NAME (1940); VANDEL (1952b); VANDEl (1953); Lemos DE CASTRO (1967); TAITI, FERRARA \& KWON (1992)

Distribution: pantropical, in America: Costa Rica; Panama, Barro Colorado; Venezuela; Brazil, Amapá

\section{Cordioniscus Graeve, 1914}

Cordioniscus leleupi Vandel, 1968

Literature: VANDEL (1968)

Distribution: Ecuador, Oriente

Cordioniscus stebbingi (Patience, 1907)

Synonymy: Trichoniscus stebbingi Patience, 1907

Literature: VAN NAME (1936); HATCH (1947); Lemos DE CASTRO (1953); VANDEL. (1953); LEMOS DE CASTRO (1971)

Distribution: Brazil, Rio de Janeiro; greenhouses in Europe and USA, Massachusetts, Oregon

\section{Kuscheloniscus Strouhal, 1961}

Kuscheloniscus vandeli Strouhal, 1961

Literature: STROUHAL (1961)

Distribution: Chile, Juan Fernandez Islands

Notoniscus Chilton, 1915

Notoniscus fernandezi Strouhal, 1961

Literature: STROUHAL (1961)

Distribution: Chile, Juan Fernandez Islands

Notoniscus secundus Strouhal, 1961

Literature: STROUHAL (1961)

Distribution: Chile, Juan Fernandez Islands

Notoniscus tertius Strouhal, 1961

Literature: STROUHAL (1961)

Distribution: Chile, Juan Fernandez Islands

Pectenoniscus Andersson, 1960

Pectenoniscus angulatus Andersson, 1960

Literature: ANDERSSON (1960)

Distribution: Brazil, Santa Catarina

Styloniscus Dana, 1853

Styloniscus araucanicus Verhoeff, 1939

Literature: VERHOEFF (1939)

Distribution: Chile, Puerto Puyuhuapi

Styloniscus iheringi Verhoeff, 1951

Literature: VERIIOEFF (1951)

Distribution: Falkland Islands 
Styloniscus magellanicus Dana, 1853

Synonymy: Trichoniscus magellanicus Giambiagi de Calabrese, 1939

Literature: VAN NAME (1936); Giambiagi de CALABRESE (1939); VAN NAME (1940); VANDEL (1952c); ANDERSSON (1960); VANDEl (1963); VAN KLINKEN \& GREeN (1992)

Distribution: Chile, Tierra del Fuego: Argentinia, Patagonia northwards to $39^{\circ} \mathrm{S}$; Auckland Islands; Chatham Islands

Stylonischus monocellatus (Dollfus, 1890)

Synonymy: Oligoniscus monocellatus (Dollfus, 1890)

Literature: VAN NAME (1936)

Distribution: Juan Fernandez Islands

Styloniscus murrayi Dollfus, 1890

Literature: VAN NAME (1936)

Distribution: Chile, Valparaiso

Styloniscus nordenskjöldi Verhoeff, 1939

Literature: VERHOEFF (1939); VAN NAME (1942)

Distribution: Argentinia, Patagonia

Styloniscus otakenssis fernandezianus Strouhal, 1961

Literature: STROUHAL (1961)

Distribution: Juan Fernandez Islands; nominate race from Neuseeland; Southwestern Australia; Macquarie Islands; Chatham Islands; Auckland Islands

Styloniscus pallidus Verhoeff, 1939

Literature: VERHOEFF (1939); VAN NAME (1942); ANDERSSON (1960); VAN KLINKEN \& GREEN (1991)

Distribution: Argentinia, Patagonia; Falkland Islands

Styloniscus romanorum Vandel, 1973

Literature: VANDEL (1973)

Distribution: westernmost Cuba

Styloniscus schwabei Verhoeff, 1939

Literature: VERHOEFF (1939); VAN NAME (1942)

Distribution: Chile, Puerto Payahuapi

Styloniscus simplex Vandel, 1981

Literature: VANDEL (1981)

Distribution: Guatemala

Styloniscus simrothi (Verhoeff, 1939)

Synonymy: Patagoniscus simrothi Verhoeff, 1939

Literature: VerHOEFF (1939); VAN NAME (1942); VANDEL (1963)

Distribution: Chile, Andes between $36^{\circ}$ und $44^{\circ} \mathrm{S}$, Juan Fernandez Islands

Stenoniscidae Budde-Lund, 1904

Metastenoniscus Paoletti \& Stinner, 1989

Metastenoniscus neotropicalis Paoletti \& Stinner, 1989

Literature: P^OLETTI \& STINNER (1989)

Distribution: Venezuela, Falcón

Revta bras. Zool. 16 (1): 1 - 72, 1999 
Stenoniscus Aubert \& Dollfus, 1890

Stenoniscus contogensis Mulaik, 1960

Literature: MULAIK (1960)

Distribution: Mexico, Quintana Roo

Stenoniscus pleonalis Aubert \& Dollfus, 1890

Literature: VANDEL (1968); SCHULTZ (1972b)

Distribution: Galapagos, Santa Cruz; Bermuda; Southern Europe

Scyphacidae Dana, 1852

Alloniscus Dana, 1856

Alloniscus mirabilis (Stuxberg, 1875)

Synonymy: Alloniscus cornutus Budde-Lund, 1885

Literature: VAN NAME (1936); SCHULTZ (1984a); GARTHWAITE et al. (1985)

Distribution: USA, California

Alloniscus perconvexus Dana, 1856

Literature: HATCH (1947); SCHULTZ (1984a); GARTHWAITE et al. (1985)

Distribution: USA, California to Canada, British Coumbia

Alloniscus salinarum Vandel, 1968

Literature: VANDEL (1968)

Distribution: Ecuador, Guayas

Alloniscus species Richardson, 1913

Literature: RicHARDSON (1913); VAN NAME (1936)

Distribution: Costa Rica

Alloniscus thalassophilus Rioja, 1964

Literature: RIOJA (1964); SCIIULTZ (1984a)

Distribution: Mexico, Guerrero

\section{Armadilloniscus Uljanin, 1875}

Armadilloniscus caraibicus Paoletti \& Stinner, 1989

Literature: PAOLETTI \& STINNER (1989)

Distribution: Venezuela, Falcón

Armadilloniscus coronacapitalis Menzies, 1950

Literature: Menzies (1950); Garthwaite et al. (1985); Garthwaite (1988); GarTHWATTE et al. (1992)

Distribution: USA, California

Armadilloniscus ellipticus (Harger, 1878)

Literature: VAN NAME (1936); SChUlTZ (1972c); GARTHWAITE et al. (1992)

Distribution: USA, Massachusetts to Florida; Bermudas

Armadilloniscus holmesi Arcangeli, 1933

Synonymy: Armadilloniscus tuberculatus Holmes \& Gay, 1909 non Dollfus, 1898

Literature: VAN NAME (1936); VAN NAME (1940); Hatch (1947); MENZIES (1950);

MULAIK (1960); SCIIULTZ (1972c); BOWMAN (1977); GARTHWAITE et al. (1985);

GarTHWAITE \& LaWSON (1992); GarTHWAITE et al. (1992)

Distribution: USA, Washington to Mexico

Armadilloniscus lindahli (Richardson, 1905)

Synonymy: Scleropactes cedrosensis Mulaik, 1960 
Literature: MenZIES (1950); MULAiK (1960); SCHUlTZ (1970d); SCHULTZ (1972c); Garthwaite et al. (1985); GarthWAite (1988); GarthWaite \& LaWson (1992); GARTHWAITE et al. (1992)

Distribution: USA, California; Mexico, Baja California

Armadilloniscus ninae Schultz, 1984

Literature: SCHULTZ (1984c)

Distribution: Belize

Armadilloniscus steptus Schotte \& Heard, 1991

Literature: SCHOTTE \& HEARD (1991)

Distribution: West Indies: Turks und Caicos Islands

Deto Guérin, 1836

Deto bucculenta (Nicolet, 1849)

Literature: VAN NAME (1936); SCHULTZ (1972c)

Distribution: Chile, Valparaiso

Deto marina (Chilton, 1884)

Literature: VAN NAME (1936); SCHULTZ (1972c)

Distribution: Falkland Islands; Australia; New Zealand

Detonella Lohmander, 1227

Detonella papillicornis Richardson, 1904

Synonymy: Detonella lomanderi Verhoeff, 1942

Literature: HATCH (1947); GARTHWAITE (1988)

Distribution: USA, Alaska, Washington; Canada, British Columbia

Scyphacella Smith, 1873

Scyphacella arenicola Smith, 1873

Literature: VAN NAME (1936); SCHULTZ (1972c)

Distribution: USA, Massachusetts to Florida

Philosciidae Vandel, 1952

Alboscia Schultz, 1995

Alboscia elongata Schultz, 1995

Literature: SCHULTZ (1995)

Distribution: Paraguay, Concepción

Andenoniscus Verhoeff, 1941

Andenoniscus silvaticus Verhoeff, 1941

Literature: VERHOEFF (1941b); LEISTIKOW (1998a)

Distribution: ?Chile, Aina

Andenoniscus tropicalis Vandel, 1968

Synonymy: Erophiloscia tropicalis (Vandel, 1968)

Literature: VANDEL $(1968,1972 a)$; LEISTIKOW (1998a)

Distribution: Ecuador, San Domingo

Remark: This species differs in many respects from the true Erophiloscia Vandel, 1972, so it is not justified to transfer it to this genus. Until the true relationships can be proved, it is better to retain it in Andenoniscus Verhoeff, 1941. 


\section{Araucoscia Verhoeff, 1939}

Araucoscia chilenica Verhoeff, 1939

Literature: VERHOEFF (1939); VAN NAME (1942); LEISTIKOW (1998a)

Distribution: Chile, Calbuco

\section{Archaeoscia Vandel, 1973}

Archaeoscia singularis Vandel, 1973

Literature: VANDEL (1973)

Distribution: Cuba, Las Villas

\section{Arhina Budde-Lund, 1904}

Remark: The ascription of this genus to Philosciidae is far from certain.

Arhina porcellioides Budde-Lund, 1904

Literature: VAN NAME (1936)

Distribution: Westindies

\section{Atlantoscia Ferrara \& Taiti, 1981}

Atlantoscia floridana (van Name, 1940)

Synonymy: Philoscia floridana van Name, 1940

Ocelloscia floridana Schultz \& Johnson, 1984

Chaetophiloscia paulensis Vandel, 1963 non Moreira, 1927

Atlantoscia alceui Ferrara \& Taiti, 1981

Literature: VAN NAME (1940); VANDEL (1963); VANDEL (1977); Ferrara \& TAiti (1981); SChultz \& JohnSon (1984); Lemos dE CASTRo (1985b); Johnson (1986); TAITI \& FERRARA (1991a); Araujo et al. (1996)

Distribution: USA, Florida; Brazil, São Paulo, Rio de Janeiro, Santa Catarina, Rio Grande do Sul; Argentinia, La Plata; Trinidad; Ascension; St. Helena

\section{Baconaoscia Vandel, 1981}

Baconaoscia negreai Vandel, 1981

Literature: VANDEL (1981)

Distribution: Cuba

\section{Benthana Budde-Lund, 1908}

Benthana albomarginata Lemos de Castro, 1958

Literature: LEMOS DE CASTRO (1958b)

Distribution: Brazil, Espírito Santo

Benthana angustata (Nicolet, 1849)

Literature: VAN NAME (1936)

Distribution: Chile

Benthana bilineata (Nicolet, 1849)

Literature: VAN NAME (1936)

Distribution: Chile

Benthana bocainensis Lemos de Castro, 1958

Literature: LEMOS DE CASTRO (1958b)

Distribution: Brazil, São Paulo

Benthana convexa Lemos de Castro, 1958

Literature: LEMOS DE CASTRO (1958b)

Distribution: Brazil, São Paulo 
Benthana dimorpha Lemos de Castro, 1985

Literature: LEMOS DE CASTRO (1985a)

Distribution: Brazil, Espírito Santo

Benthana iporangensis Lima \& Serejo, 1993

Literature: LIMA \& SEREJO (1993)

Distribution: Southeastern Brazil, São Paulo

Benthana longicornis Verhoeff, 1941

Literature: VERHOEFF (1941 c); GRUNER (1955); LEMOS DE CASTRO (1958b); ANDERSSON (1960); ARAuJo et al. (1996)

Distribution: Brazil, Santa Catarina

Benthana longipenis Lemos de Castro, 1958

Literature: LEMOS DE CASTRO (1958b)

Distribution: Brazil, São Paulo

Benthana moreirai Lemos de Castro, 1985

Literature: LEMOS DE CASTRO (1985a)

Distribution: Brazil, São Paulo

Benthana olfersi (Brandt, 1833)

Synonymy: Philoscia olfersi Brandt, 1833

Halophiloscia brasiliensis Moreira, 1932

Oniscus nigrescens Dana, 1852

Literature: VAN NAME (1936); VERHOEFF (1941c); GRUNER (1955); LEMOS DE CASTRO (1958b)

Distribution: Brazil, Rio de Janeiro, São Paulo

Benthana peruensis Gruner, 1955

Literature: GRUNER (1955)

Distribution: Peru

Benthana picta (Brandt, 1833)

Literature: VAN NAME (1936); CAMARgo (1954); Lemos de CASTRo (1958b); VANDEL (1963); SChUltz (1995); ARAujo et al. (1996)

Distribution: Brazil, Rio de Janeiro to Rio Grande do Sul; Paraguay; Argentinia

Benthana santosi Lemos de Catro, 1958

Literature: LEMOS DE CASTRO (1958b)

Distribution: Brazil, Rio de Janeiro, São Paulo, Minas Gerais

Benthana schubarti Lemos de Castro, 1958

Literature: LEMOS DE CASTRO (1958b)

Distribution: Brazil, Districto Federal, São Paulo

Benthana sulcata Gruner, 1955

Literature: GRUNER (1955); LEMOS DE CASTRO (1958b)

Distribution: Brazil, Rio de Janeiro

Benthana taeniata Araujo \& Buckup, 1994

Literature: ARAUJO \& BUCKUP (1994a)

Distribution: Brazil, Rio Grande do Sul, Santa Catarina

Benthana villosa (Jackson, 1926)

Literature: VAN NAME (1936); GRUNER (1955); Lemos de CASTRo (1958b)

Distribution: Peru, Matucana

Revta bras. Zool. 16 (1): 1 - 72, 1999 
Benthana werneri Lemos de Castro, 1958

Literature: LEMOS DE CASTRO (1958b)

Distribution: Brazil, São Paulo

Benthanoides Lemos de Castro, 1958

Benthanoides pauper (Jackson, 1926)

Literature: VAN NAME (1936); GRUNER (1955); LEMOS DE CASTRO (1958b)

Distribution: Chile, Valparaiso

Benthanoscia Lemos de Castro, 1958

Benthanoscia longicaudata Lemos de Castro, 1958

Literature: LEMOS DE CASTRO (1958c)

Distribution: Brazil, Rio de Janeiro

Burmoniscus Collinge, 1914

Burmoniscus meeusei (Holthuis, 1946) \#

Literature: ARAujo et al. (1996)

Distribution: Brazil, Santa Catarina

Native distribution: Southeastern Asia

Caraiboscia Vandel, 1968

Caraiboscia microphthalma Vandel, 1968

Literature: VANDEL (1968)

Distribution: Galapagos

Chaetophiloscia Verhoeff, 1908

This genus is of westpalaearctic distribution and the American members have to be re-examinated in the sense of phylogenetic systematics.

Chaetophiloscia frontalis Lemos de Castro, 1967

Literature: LEMOS DE CASTRO (1967)

Distribution: Brazil, Pará

Chaetophiloscia gatunensis (van Name, 1926)

Synonymy: Philoscia gatunensis van Name, 1926

Literature: VAN NAME (1926); ARCANGeli (1930); VAN NAME (1936); Lemos de CASTRO (1967)

Distribution: Costa Rica; Panama; Brazil, Amazonas, Pará

Chaetophiloscia species Vandel, 1963

Literature: VANDEL (1963)

Distribution: Brazil, Pernambuco

Chaetophiloscia walkeri (Pearse, 1915)

Synonymy: Philoscia walkeri Pearse, 1915

Literature: VAN NAME (1936); LEMOS DE CASTRO (1967)

Distribution: Colombia, Santa Marta; Brazil, Pará

Colombophiloscia Vandel, 1968

Remark: Beside the three species recorded from the northern South America and Galapagos, a species from Cuba is named Colombophiloscia, too. It was introduced as genus novum and species nova by VANDEL (1981). It seems that there is a case of homonymy and Colombophiloscia romanorum Vandel, 1981 has to be removed from this genus. Therefore, a re-examination of the whole genus is necessary. 
Colombophiloscia alticola Vandel, 1968

Literature: VANDEL (1968)

Distribution: Ecuador, Banos

Colomobophiloscia cavernicola Vandel, 1968

Literature: VANDEL (1968); LEISTIKOW (1998b)

Distribution: Venezuela, Monaguas

Colombophiloscia naevigesta Vandel, 1968

Literature: VANDEL (1968)

Distribution: Galapagos, Sta. Cruz

Colombophiloscia romanorum Vandel, 1981

Literature: VANDEL (1981)

Distribution: Cuba

Cubanophiloscia Vandel, 1973

Cubanophiloscia briani (Arcangeli, 1929)

Synonymy: Philoscia briani ARCANGeli, 1929

Literature: VAN NAME (1936); VANDEL (1973)

Distribution: Cuba

Ecuadoroniscus Vandel, 1968

Ecuadoroniscus orientalis Vandel, 1968

Literature: VANDEL (1968)

Distribution: Ecuador, Oriente

Erophiloscia Vandel, 1972

Erophiloscia longistyla Vandel, 1972

Literature: VANDEL (1972a)

Distribution: Colombia, Montserrate, Bogotá

Erophiloscia narcissi (Vandel, 1968)

Synonymy: Andenoniscus narcissi Vandel, 1968

Literature: VANDEL (1968); VANDEL (1972a)

Distribution: Ecuador, Oriente

Floridoscia Schultz \& Johnson, 1984

Floridoscia fusca Schultz \& Johnson, 1984

Literature: SCHULTZ \& JOHNSON (1984); JOHNSON (1986)

Distribution: USA, Florida

Hoctunus Mulaik, 1960

Hoctunus vespertillo Mulaik, 1960

Literature: MULAIK (1960)

Distribution: Mexico, Yucatán

Ischioscia Verhoeff, 1928

Ischioscia amazonica Lemos de Castro, 1955

Synonymy: Proischioscia amazonica Vandel, 1968

Literature: Lemos DE CASTRo (1955); Lemos DE CASTRo (1967); VANDEl (1968); SCHMALFUSS (1980a)

Distribution: Brazil, Amazonia 
Ischioscia andina (Vandel, 1968)

Synonymy: Proischioscia andina Vandel, 1968

Literature: VANDEL (1968); SCHMALFUSS (1980a)

Distribution: Ecuador, Cotopaxi

Ischioscia bolivari Vandel, 1968

Literature: VANDEL (1968); SCHMALFusS (1980a)

Distribution: Ecuador, Santo Domingo

Ischioscia elongata Leistikow, 1997

Synonymy: Ischioscia variegata non Dollfus, 1896

Literature: ARCANGELI (1930); VAN NAME (1936); LEISTIKOW (1997a)

Distribution: Costa Rica

Ischioscia hanagarthi Schmalfuss, 1980

Literature: SCHMALFUSS (1980a)

Distribution: Peru, Huanuco

Ischioscia irmleri Schmalfuss, 1980

Literature: SCHMALFUSS (1980a)

Distribution: Brazil, Amazonas

Ischioscia longicauda Schmalfuss, 1980

Literature: SCHMALFUSS (1980a)

Distribution: Peru, Huanuco

Ischioscia martinae Leistikow, 1997

Literature: LEISTIKOW (1997a)

Distribution: Costa Rica, Cordilleras de Talamanca and Tilarán

Ischioscia mineri van Name, 1936

Synonymy: Philoscia (Ischioscia) mineri van Name, 1936

Literature: VAN NAME (1936); VAN NAME (1940); SCHMALFuss (1980a)

Distribution: Dominica, Guadeloupe

Ischioscia muelleri Leistikow, 1997

Synonymy: Philoscia muscorum non (Scopoli, 1793)

Literature: RICHARDSON (1910); LEISTIKOW (1997a)

Distribution: Costa Rica

Ischioscia nitida (Miers, 1877)

Synonymy: Philougria nitida Miers, 1877

Literature: VAN NAME (1936); SCHMALFuSS (1980a)

Distribution: Peru, Guiana

Ischioscia stenocarpa Schmalfuss, 1980

Literature: SCHMALFUSS (1980a)

Distribution: Peru, Huanuco

Ischioscia sturmi (Vandel, 1972)

Synonymy: Proischioscia sturmi Vandel, 1972

Literature: VANDEL (1972a); SCHMALFUSS (1980a)

Distribution: Colombia, Bogotá, La Guayacana

Ischioscia variegata (Dollfus, 1896)

Synonymy: Ischioscia lobifera Verhoeff, 1928

Literature: Dollfus (1896c); VerhoEfF (1928); VAN NAME (1926); ARCANGELI (1930); 
Arcangeli (1932); Van Name (1936); Verhoeff (1941b); Paulian de Félice (1944); VANDEL (1952b); SChMALFUSS (1980a); LEISTIKOW (1997a)

Distribution: Venezuela, Macay; Dominica (?); French Guiana (?)

$$
\text { Jimenezia Vandel, } 1973
$$

Jimenezia heteroclita Vandel, 1973

Literature: VANDEL (1973);

Distribution: Cuba, Oriente

\section{Littorophiloscia Hatch, 1947}

Littorophiloscia alticola (Vandel, 1977)

Synonymy: Helenoscia alticola Vandel, 1977

Literature: VANDEL (1977); TAITI \& FERRARA (1986)

Distribution: St. Helena

Littorophiloscia bermudensis (Dahl, 1892)

Literature: VAN NAME (1936)

Distribution: Bermuda

Littorophiloscia culebrae (Moore, 1901)

Synonymy: Philoscia miamensis SchUlTZ, 1966

Literature: Van Name (1936); Schultz (1966); Lemos de Castro (1968a); Taiti \& FERRARA (1986); JoInSON (1986); MUCHMORE (1993)

Distribution: USA, Florida, Virgin Islands, Hawaii; Puerto Rico; Cuba; Angola; Madagascar

Littorophiloscia nomae (van Name, 1924)

Literature: VAN NAME (1936)

Distribution: Galapagos

Littorophiloscia richardsonae (Holmes \& Gay, 1909)

Synonymy: Philoscia richardsonae Holmes \& Gay, 1909

Literature: VAN NAME (1936); VAN NAME (1940); HATCH (1947); Mulaik (1960); Lemos de Castro (1968); Bowman (1977); GarThWAITE et al. (1985); TAITI \& FERRARA (1986); GARTHWAITE (1992)

Distribution: USA, Washington, California; Mexico, Baja California

Littorophiloscia tropicalis Taiti \& Ferrara, 1986

Synonymy: Alloniscus compar Vandel, 1953 non Budde-Lund, 1893

Vandeloscia riedli SCHULTZ, 1983

Literature: VAndel (1977); Schultz (1983a); TAitI \& Ferrara (1986); TAitI \& FERRARA (1991)

Distribution: USA, Florida; Mexico; Belize; Venezuela; Brazil; St. Helena; Ascension, Cameroon; Somalia; Sudan; India?

Littorophiloscia vittata (Say, 1818)

Synonymy: Philoscia vittata Say, 1818

Philoscia robusta Schultz, 1963

Sayoscia vittata Schultz, 1983

Literature: VAN NAme (1936); Lemos de CAStro (1968a); Schultz (1963b); Schultz (1974a); SCHultz (1977a); SChultz (1983a); TAitl \& FERRARA (1986); Johnson (1986); JASS \& KLAUSMEIER (1990)

Distribution: USA, Great Lakes, Georgia, Florida 
Microphiloscia Vandel, 1973

Microphiloscia trichoniscoides Vandel, 1973

Literature: VANDEL (1973)

Distribution: Cuba, Oriente

Mirtana Leistikow, 1997

Mirtana costaricensis Leistikow, 1997

Literature: LEISTIKOW (1997b)

Distribution: Costa Rica, San José

Nesophiloscia Vandel, 1968

Nesophiloscia culebroides (van Name, 1936)

Synonymy: Philoscia culebroides van Name, 1936

Literature: VAN NAME (1936); VANDEL (1968)

Distribution: Galapagos

Oniscophiloscia Wahrberg, 1922

Oniscophiloscia anomala (Dollfus, 1890)

Synonymy: Philoscia anomala Dollfus, 1890

Phalloniscus anomalus Budde-Lund, 1885 partim

Literature: VAN NAME (1936); Lemos de CASTRo (1960); STrouhal (1961)

Distribution: Chile, Juan Fernandez Islands

Oniscophiloscia kuscheli Strouhal, 1961

Literature: STROUHAL (1961)

Distribution: Chile, Juan Fernandez Islands

Oniscophiloscia mirifica Wahrberg, 1922

Literature: VAN NAME (1936); STROUHAL (1961)

Distribution: Chile, Juan Fernandez Islands, adjacent coasts (?)

Oreades Vandel, 1968

Oreades lativentris Vandel, 1968

Literature: VANDEL (1968)

Distribution: Ecuador, Oriente

Pacroscia Vandel, 1981

Pacroscia decoui Vandel, 1981

Literature: VANDEL (1981)

Distribution: Cuba

Pacroscia elongata Vandel, 1981

Literature: VANDEL (1981)

Distribution: Cuba

Paraguascia Schultz, 1995

Paraguascia pigmentata Schultz, 1995

Literature: SCHULTZ (1995)

Distribution: Paraguay, Misiones

Parapacroscia Vandel, 1981

Parapacroscia negreai Vandel, 1981

Literature: VANDEL (1981)

Distribution: Cuba 


\section{Parischioscia Lemos de Castro, 1967}

Parischioscia omissa (van Name, 1936)

Synonymy: Philoscia omissa van Name, 1936

Literature: Van Name (1936); Paulian de Félice (1944); Lemos de Castro (1967)

Distribution: Guyana, French Guiana; Brazil, Amapá

\section{Pentoniscus Richardson, 1913}

Remark: SCHULTZ (1968) states that in contrast to RICHARDSON's diagnosis (RICHARDSON 1913), the flagellum of the antenna is composed of only three articles. Therefore, he synonymizes this genus with Philoscia Latreille, 1804. A revision of the type material has proved the validity of this genus (LEISTIKOW 1998b)

Pentoniscus dominicensis Arcangeli, 1932

Synonymy: Philoscia dominicensis Schultz, 1968

Literature: ARCANGELI (1932); VAN NAME (1936); SCHULTZ (1968); LEISTIKOW (1998b)

Distribution: Dominica

Pentoniscus exilis van Name, 1925

Synonymy: Philoscia exilis Schultz, 1968

Literature: VAN NAME (1936); SchultZ (1968); Leistikow (1998b)

Distribution: Guiana, Kartabo

Pentoniscus pruinosus Richardson, 1913

Synonymy: Philoscia pruinosa Schultz, 1968 non Carl, 1908

Literature: Richardoson (1913); ArCangeli (1930); Van Name (1936); Schultz (1968); LEISTIKOW (1998b)

Distribution: Costa Rica

Pentoniscus vargasae Leistikow, 1998

Literature: LEISTIKOW (1998b)

Distribution: Costa Rica, San José

\section{Philoscia Latreille, 1804}

Remark: Apart from the introduced European species Philoscia muscorum (Scopoli, 1793), it is doubtful if there are native species of this genus in America. It is more probable that a revision of the American species ascribed to the genus Philsocia Latreille, 1804, which throughout are only superficially described, will prove their membership to other genera.

Philoscia bonarensis (Giambiagi de Calabrese, 1935)

Literature: GlambiaGi de CALABRESE (1935); VAN NAME (1940)

Distribution: Argentinia, Buenos Aires

Philoscia colimensis Mulaik, 1960

Literature: MULAIK (1960)

Distribution: Mexico, Colima

Philoscia contogensis Mulaik, 1960

Literature: MULAIK (1960)

Distribution: Mexico, Qunintana Roo

Philoscia ctenoscoides Mulaik, 1960

Literature: MULAIK (1960)

Distribution: Mexico, Chiapas, Tabasco, Quintana Roo 
Philoscia demerarae van Name, 1925

Literature: VAN NAME (1936)

Distribution: Guyana

Philoscia diminuta Budde-Lund, 1893

Literature: VAN NAME (1936)

Distribution: Venezuela, Caracas, La Moka

Philoscia formosae Mulaik, 1960

Literature: MULAIK (1960)

Distribution: Mexico, Tabasco

Philoscia geayi Paulian de Félice, 1944

Literature: PAULIAN DE FÉLICE (1944)

Distribution: French Guiana

Philsocia geiseri van Name, 1936

Literature: VAN NAME (1936)

Distribution: USA, Texas

Philoscia gracilior Paulian de Félice, 1944

Literature: PAULIAN DE FÉLICE (1944)

Distribution: French Guiana

Philoscia guerrerense Mulaik, 1960

Literature: MULAIK (1960)

Distribution: Mexico, Guerrero

Philoscia incerta Arcangeli, 1932

Literature: ARCANGELI (1932); VAN NAME (1936)

Distribution: Dominica; Guadeloupe

Philoscia inquilina van Name, 1936

Literature: VAN NAME (1936)

Distribution: Guayana

Philoscia kartaboana van Name, 1936

Literature: VAN NAME (1936)

Distribution: Guayana

Philoscia moneaguensis van Name, 1936

Literature: VAN NAME (1936)

Distribution: Jamaica, Moneague

Philoscia muscorum (Scopoli, 1793) \#

Literature: VAN NAME (1936); HATCH (1947); SChULTZ (1965a); JASS \& KLAUSMEIER (1990)

Distribution: USA, New York, Washington, Massachusetts

Native distribution: West and Central Europe

Philoscia richmondi Richardson, 1901

Literature: VAN NAME (1936)

Distribution: Puerto Rico

Philoscia roraimae van Name, 1936

Literature: VAN NAME (1936)

Distribution: Venezuela, Roraima 
Philoscia seriepunctata Budde-Lund, 1885

Literature: VAN NAME (1936)

Distribution: Venezuela, Caracas

Philoscia spinosa Say, 1818

Literature: VAN NAME (1936)

Distribution: USA, Georgia

Philoscia veracruzana Mulaik, 1960

Literature: MULAIK (1960)

Distribution: Mexico, Veracruz

Prosekia Vandel, 1968

Prosekia albamaculata Lima, 1996

Literature: LiMA (1996b)

Distribution: Brazil, Amazonas

Prosekia galapagensis (Andersson, 1960)

Synonymy: Chaetophilsocia galapagensis Andersson, 1960

Literature: ANDERSSON (1960); VANDEL (1968)

Distribution: Galapagos

Prosekia hamigera (Vandel, 1952)

Synonymy: Chaetophilsocia hamigera Vandel, 1952

Literature: VANDEL (1952b); VANDEL (1968)

Distribution: Venezuela

Prosekia insularis Lemos de Castro \& Souza, 1986

Literature: LEMOS DE CASTRO \& SOUZム (1986)

Distribution: Brazil, Pará

Prosekia lejeunei Lemos de Castro \& Souza, 1986

Literature: LEMOS DE CASTRO \& SOUZA (1986)

Distribution: Brazil, Pará

Prosekia pearsei (Vandel, 1952)

Synonymy: Chaetophiloscia pearsei Vandel, 1952

Literature: VANDEL (1952b); VANDEL (1968)

Distribution: Venezuela

Prosekia rutilans (Vandel, 1952)

Synonymy: Chaetophiloscia rutilans Vandel, 1952

Literature: VANDEL (1952b); VANDEL (1968)

Distribution: Venezuela

Prosekia silvatica Lemos de Castro \& Souza, 1986

Literature: LEMOS DE CASTRO \& SOUZA (1986)

Distribution: Brazil, Amazonas

Prosekia species (Vandel, 1952)

Synonymy: Chaetophiloscia species Vandel, 1952

Literature: VANDEL (1952b); VANDEL (1968)

Distribution: Venezuela

Prosekia tarumae Lemos de Castro, 1984

Literature: LEMOS DE CASTRO (1984a)

Distribution: Brazil, Amazonas 
Pseudophiloscia Budde-Lund, 1904

Pseudophiloscia angusta (Dana, 1852)

Literature: VAN NAME (1936); LeIsTIKOW (1998c)

Distribution: Chile, Tierra del Fuego

Pseudophiloscia inflexa Budde-Lund, 1904

Literature: VAN NAME (1936); LeISTIKOW (1998c)

Distribution: Chile, Corral

Puteoscia Vandel, 1981

Puteoscia silvestrii Vandel, 1981

Literature: VANDEL (1981)

Distribution: Cuba

Rostrophiloscia Arcangeli, 1932

Rostrophiloscia dominicensis Arcangeli, 1932

Literature: ARCANGELI (1932); VAN NAME (1936)

Distribution: Dominica

Suleoscia Vandel, 1973

Suleoscia epigea Vandel, 1973

Literature: VANDEL (1973)

Distribution: Cuba, Oriente

Thomasoniscus Vandel, 1981

Thomasoniscus angulatus Vandel, 1981

Literature: VANDEL (1981)

Distribution: Cuba

Troglophiloscia Brian, 1929

Troglophiloscia belizensis Schultz, 1984

Literature: SCHULTZ (1984c); LEISTIKOW (1998b)

Distribution: Belize

Troglophiloscia laevis Schultz, 1977

Literature: SCIIULTZ (1977b); LEISTIKOW (1998b)

Distribution: Mexico, Yucatán

Troglophiloscia silvestrii Brian, 1929

Literature: VAN NAME (1936); RIOJA (1956); VANDEL (1973); SCHULTZ (1981)

Distribution: Cuba, Matanzas, La Habana

Troglophiloscia species Rioja, 1956

Literature: RIOJA (1956)

Distribution: Cuba, La Habana

Tropiscia Vandel, 1968

Tropiscia flagellata Vandel, 1968

Literature: VANDEL (1968)

Distribution: Ecuador, Oriente

Xiphoniscus Vandel, 1968

Xiphoniscus mirabilis Vandel, 1968

Literature: VANDEL (1968)

Distribution: Ecuador, Oriente, Banos 


\section{Halophilosciidae Verhoeff, 1908 \\ Halophiloscia Verhoeff, 1908}

Halophiloscia couchii (Kinahan, 1858) \#

Synonymy: Halophiloscia brasiliensis Moreira, 1932

Literature: VAn NAme (1936); Lemos de CASTRo (1958d); Lemos de Castro (1968a); RECA (1972); SCHULTZ 1972b)

Distribution: USA, Virginia; Bermudas; Brazil; Argentinia, Buenos Aires

Native distribution: Atlantic coasts of Europe

Oniscidae Latreille, 1806

Oniscus Linné, 1758

Oniscus armatus Nicholet, 1849

Literature: VAN NAME (1936)

Distribution: Chile

Oniscus asellus Linné, 1758 \#

Literature: VAN NAME (1936); HATCH (1947); PALMÉN (1951); CAUSEy (1952); MULAIK (1960); Vandel (1977); Zardo \& Loyola e Silva (1988); Jass \& Klausmeier (1990); SNIDER (1991); JAsS \& KLAUSMEIER (1996)

Distribution: Canada, Newfoundland; USA, eastern states, Arkansas; Mexico; Brazil; St. Helena;

Native distribution: Europe

\section{Dubioniscidae Schultz, 1995 \\ Calycuoniscus Collinge, 1915}

Calycuoniscus bodkini Collinge, 1915

Literature: VAN NAME (1936); LEMOS DE CASTRO (1967)

Distribution: Brazil, Pará

Calycuoniscus compar (Budde-Lund, 1893)

Synonymy: Alloniscus compar Budde-Lund, 1893

Literature: VAN NAME (1936); TAITI \& FERRARA (1986)

Distribution: Venezuela, Caracas, La Moka

Calycuoniscus spinosus Collinge, 1918

Literature: VAN NAME (1936)

Distribution: Trinidad

Dubioniscus Vandel, 1963

Dubioniscus delamarei Vandel, 1963

Literature: VANDEL (1963); VANDEL (1972b)

Distribution: Argentinia, La Plata; Brazil; Paraguay, Misiones

Dubioniscus goeldii (Lemos de Castro, 1967)

Synonymy: Hileioniscus goeldii Lemos de Castro, 1967

Calycuoniscus goeldii Lemos de Castro, 1968

Literature: Lemos DE CASTRO (1967); LeMOS DE CASTRO (1968b); SCHULTZ (1995)

Distribution: Brazil, Pará

Dubioniscus insularis Vandel, 1972

Literature: VANDEL (1972b); VANDEL (1973)

Distribution: Cuba

Revta bras. Zool. 16 (1): 1 - 72, 1999 
Dubioniscus marmoratus Lemos de Castro, 1970

Literature: LEMOS DE CASTRO (1970a)

Distribution: Brazil, Rio de Janeiro

Dubioniscus negreae Vandel, 1973

Literature: VANDEL (1973)

Distribution: Cuba, Pinar del Río

Novamundoniscus Schultz, 1995

Novamundoniscus dissimilis (Lemos de Castro, 1960)

Synonymy: Phalloniscus dissimilis Lemos de Castro, 1960

Literature: LEMOS DE CASTRO (1960); SCHULTZ (1995)

Distribution: Brazil, Rio de Janeiro

Novamundoniscus macrophthalmus (Lemos de Castro, 1960)

Synonymy: Phalloniscus macrophthalmus Lemos de Castro, 1960

Literature: LEMOS DE CASTRo (1960); SChUlTZ (1995)

Distribution: Brazil, Rio de Janeiro

Novamundoniscus marcuzzi (Vandel, 1952)

Synonymy: Phalloniscus marcuzzi Vandel, 1952

Literature: VANDEL (1952b); LEMOS DE CASTRO (1960); SCHULTZ (1995)

Distribution: Venezuela, Caracas

Novamundoniscus persimilis (Vandel, 1952)

Synonymy: Phalloniscus persimilis Vandel, 1952

Literature: VANDEL (1952b); LEMOS DE CASTRO (1960); LEMOS DE CASTRO (1967); SCHULTZ (1995)

Distribution: Venezuela, Tunapuncito; Brazil, Pará

Novamundoniscus singularis (Lemos de Castro, 1967)

Synonymy: Phalloniscus singularis Lemos de Castro, 1967

Literature: LEMOS DE CASTRO (1967); SCHULTZ (1995)

Distribution: Brazil, Amazonas

Novamundoniscus vandeli (Lemos de Castro, 1960)

Synonymy: Phalloniscus vandeli Lemos de Castro, 1960

Literature: LEMOS DE CASTRO (1960); SCHULTZ (1995)

Distribution: Brazil, Minas Gerais, Rio de Janeiro

\section{Phalloniscus Budde-Lund, 1908}

Remark: According to Scilultz (1995), all the American species of Phalloniscus Budde-Lund, 1908 should be regarded as members of Novamundoniscus Schultz, 1995. The ascription of the following species seems to be doubtful:

Phalloniscus avrilensis (van Name, 1936)

Synonymy: Philoscia avrilensis van Name, 1936

Literature: VAN NAME (1936); LEMOS DE CASTRO (1960)

Distribution: Haiti, Bois d'Avril

Phalloniscus baldoni (Arcangeli, 1930)

Synonymy: Philoscia baldoni ARCANGel, 1930

Literature: ARCANGELI (1930); VAN NAME (1936); SChUltZ (1995)

Distribution: Costa Rica, San José 
Phalloniscus barbouri (van Name, 1926)

Synonymy: Trichorhina barbouri van Name, 1926

Literature: VAN NAME (1926); LEMOS DE CASTRO (1967); SCHULTZ (1995)

Distribution: Panama; Brazil

Phalloniscus langi (van Name, 1936)

Synonymy: Philoscia langi van Name, 1936

Literature: VAN NAME (1936); SCHULTZ (1995)

Distribution: Guiana, Kamakusa

Phalloniscus loyolai Zardo, 1989

Literature: ZARDO (1989)

Distribution: Brazil, Paraná

Phalloniscus meridionalis Araujo \& Buckup, 1994

Literature: ARAUJO \& BUCKUP (1994a)

Distribution: Brazil, Santa Catarina, Rio Grande do Sul

Phalloniscus pearsei (van Name, 1936)

Synonymy: Philoscia pearsei van Name, 1936

Literature: VAN NAME (1936); SCHULTZ (1995)

Distribution: Guiana, Dunoon

Phalloniscus setosus Lemos de Castro, 1960

Literature: LEMOS DE CASTRO (1960)

Distribution: Brazil, Minas Gerais

Bathytropidae Vandel, 1952

Cubanoscia Vandel, 1981

Cubanoscia primitiva Vandel, 1981

Literature: VANDEL (1981)

Distribution: Cuba

Cubanoscia proxima Vandel, 1981

Literature: VANDEL (1981)

Distribution: Cuba

Cubanoscia romanorum Vandel, 1981

Literature: VANDEL (1981)

Distribution: Cuba

Laninoniscus Reca, 1973

Laninoniscus giambiagiae Reca, 1973

Literature: RECA (1973)

Distribution: Argentinia, Neuquén

Neotroponiscus Arcangeli, 1936

Neotroponiscus argentinus (Giambiagi de Calabrese, 1939)

Synonymy: Porcellio argentinus Giambiagi de Calabrese, 1939

Brasilocellio nodulosus Verhoeff, 1941

Literature: Giambiagi de Calabrese (1939); Verhoeff (1941c); Van NAME (1942); ANDERSSON (1960); VANDEL (1963); LEMOS DE CASTRO (1970e)

Distribution: western South America from Brazil, Pernambuco to Argentinia, La Plata 
Neotroponiscus caroli Arcangeli, 1936

Literature: ARCANGeli (1936); VAN NAme (1940); Lemos de CAstro (1970e)

Distribution: Brazil, São Paulo to Bahía

Neotroponiscus daguerrei (Giambiagi de Calabrese, 1939)

Synonymy: Porcellio daguerrei Giambiagi de Calabrese, 1939

Literature: Glambiagi de CAlabrese (1939); Van NAme (1942); Lemos de CASTro (1970d); RECA (1973); ARAujo et al. (1996)

Distribution: Argentinia, Buenos Aires; Brazil, Rio Grande do Sul

Neotroponiscus lenkoi Lemos de Castro, 1970

Literature: LEMOS DE CASTRO (1970d)

Distribution: Brazil, São Paulo

Neotroponiscus littoralis Lemos de Castro, 1970

Literature: LEMOS DE CASTRO (1970d)

Distribution: Brazil, Rio de Janeiro

Neotroponiscus lobatus Lemos de Castro, 1970

Literature: LEMOS DE CASTRO (1970d)

Distribution: Brazil, Espírito Santo

Neotroponiscus perlatus Lemos de Castro, 1970

Literature: LEMOS DE CASTRO (1970d)

Distribution: Brazil, Espírito Santo

Neotroponiscus plaumanni (Andersson, 1960)

Synonymy: Brasilocellio plaumanni ANDERSSON, 1960

Literature: ANDERSSON (1960); LEMOS DE CASTRO (1970d)

Distribution: Southern Brazil; Santa Catarina; Uruguay

Neotroponiscus vedadoensis (Boone, 1918)

Synonymy: Leptotrichus vedadoensis Boone, 1918

Literature: VAN NAME (1936); LEMOS DE CASTRO (1970e)

Distribution: Cuba, La Habana

Rhabdoniscus Vandel, 1981

Rhabdoniscus robustus Vandel, 1981

Literature: VANDEL (1981)

Distribution: Cuba

Remark: VANDEL (1981) associates this new genus provisorically with Bathytropidae, until the exact relationships are known.

\section{Platyarthridae Verhoeff, 1949}

Niamba Budde-Lund, 1908

Niamba capensis (Dollfus, 1895) \#

Synonymy: Porcellio littorinus Miller, 1936

Mauritaniscus littorinus Schultz et al., 1982

Literature: VAN NAME (1940); VANDEL (1977); SChUlTZ et al. (1982); GARTHWATTE et al. (1985), Ferrara \& TAITI (1989); GARTHWAITE \& LAWSON (1992)

Distribution: USA, California; St. Helena

Native distribution: South Africa 
Niamba duffreyi Ferrara \& Taiti, 1981

Literature: FERRARA \& TAITI (1981); TAITI \& FERRARA (1991a)

Distribution: Ascension

Niamba longiantennata Taiti \& Ferrara, 1991

Literature: TAITI \& FERRARA (1991a)

Distribution: Ascension

Niamba squamata (Budde-Lund, 1885) \# (?)

Synonymy: Leptotrichus squamatus Budde-Lund, 1885

Literature: LEMOS DE CASTRO (1967)

Distribution: Brazil, Belém, Pará

Native distribution: South Africa

\section{Platyarthrus Brandt, 1833}

Platyarthrus aiasensis Legrand, 1954 \#

Synonymy: Platyarthrus schoeblii aiasensis Legrand, 1954

Literature: GARTHWAITE \& TAITI (1989)

Distribution: South Africa, USA, California, Texas, St. Barthelemy

Native distribution: Mediterranean area

Platyarthrus hoffmannseggi Brandt, 1833 \#

Literature: VAN NAME (1940)

Distribution: northeastern USA

Native distribution: Europe

Trichorhina Budde-Lund, 1908

Trichorhina acuta Araujo \& Buckup, 1994

Literature: ARAUJO \& BUCKUP (1994b)

Distribution: Brazil, Rio Grande do Sul, Santa Catarina

Trichorhina amazonica Souza-Kury, 1997

Literature: SOUZA-KURY (1997a)

Distribution: Brazil, Pará

Trichorhina ambigua (Budde-Lund, 1893)

Literature: VAN NAME (1936)

Distribution: Venezuela, Caracas, La Moka

Trichorhina argentina Vandel, 1963

Literature: VANDEL (1963); ARAUJO \& BUCKUP (1996a)

Distribution: Argentinia, La Plata; Brazil, Rio Grande do Sul, Santa Catarina

Trichorhina atoyacensis Mulaik, 1960

Literature: MULAIK (1960); LEMOS DE CASTRO (1964)

Distribution: Mexico, Veracruz

Trichorhina bequaerti van Name, 1936

Literature: VAn NAme (1936); Lemos de CASTRO (1964); VANDEl (1973);

Distribution: Cuba, Oriente

Trichorhina bicolor Araujo \& Buckup, 1996

Literature: ARAUJO \& BUCKUP (1996a)

Distribution: Brazil, Santa Catarina

Revta bras. Zool. 16 (1): 1 - 72, 1999 
Trichorhina boliviana (Vandel, 1952)

Synonymy: Phalloniscus bolivianus Vandel, 1952

Literature: VANDEL (1952a); VANDEL (1956)

Distribution: Bolivia, Cochabamba

Trichorhina boneti Rioja, 1955

Literature: RioJA (1955a), MULAIK (1960), LEMOS DE CASTRo (1964)

Distribution: Mexico, San Luis Potosi, Xilitla

Trichorhina brasiliensis Andersson, 1960

Literature: ANDERSSON (1960); SCHULTZ (1995); ARAUJO \& BUCKUP (1996a)

Distribution: Brazil, Santa Catarina; Paraguay

Trichorhina caeca Vandel, 1952

Literature: VANDEL (1952b)

Distribution: Venezuela, El Junquito

Trichorhina donaldsoni Schultz, 1963

Literature: SCHULTZ (1963c)

Distribution: USA, Florida

Trichorhina gianelli Arcangeli, 1929

Literature: ARCANGELI (1930); VAN NAME (1936)

Distribution: Costa Rica

Trichorhina guanophila Souza-Kury, 1993

Literature: SOUZA-KURY (1993)

Distribution: Brazil, Penambuco

Trichorhina heterophthalma Lemos de Castro, 1964

Literature: Lemos DE CASTRO (1964); VANDEL (1968); VANDEL (1973); SCHULTZ (1975); BOWMAN (1977); TAITI et al. (1992); MUCHMORE (1993); SOUZA-KURY (1993)

Distribution: pantropic, in America: USA, Georgia; Cuba; Virgin Islands; Galapagos Islands, Clipperton Island, Venezuela; Brazil, Bahia, Rio de Janeiro

Trichorhina isthmica (van Name, 1926)

Literature: VAN NAME (1936)

Distribution: Panama

Trichorhina macrops Souza-Kury, 1993

Literatur: SOUZA-KURY (1993)

Distribution: Brazil, Pernambuco

Trichorhina macrophthalma Mulaik, 1960

Literature: MULAIK (1960)

Distribution: Mexico, Tabasco

Trichorhina mariani Arcangeli, 1930

Literature: ARCANGELI (1930); VAN NAME (1936)

Distribution: Costa Rica

Trichorhina paraensis Souza-Kury, 1997

Literature: SOUZA-KURY (1997a)

Distribution: Brazil, Pará

Trichorhina papillosa (Budde-Lund, 1893)

Literature: DOLLfuS (1896c); VAN NAME (1936)

Distribution: Venezuela, Los Tejes 
Trichorhina pearsei (Creaser, 1939)

Synonymy: Porcellio pearsei Creaser, 1939

Trichorhina yucatanensis Mulaik, 1960

Literature: Mulaik (1960); LEMOS DE CASTRO (1964); SOUZA-KuRY (1993)

Distribution: Mexico, Yucatán; Brazil

Trichorhina pittieri (Pearse, 1921)

Literature: VAN NAME (1936); Lemos DE CASTRO (1967)

Distribution: Venezuela; Guyana; Brazil, Pará

Trichorhina quisquiliarum (Budde-Lund, 1893)

Literature: VAN NAME (1936)

Distribution: Venezuela, Las Trincheras, La Moka

Trichorhina simoni (Dollfus, 1896)

Literature: DOLLFUS (1896c); VAN NAME (1936)

Distribution: Venezuela, Colonie Tovar

Trichorhina squamata (Verhoeff, 1933)

Synonymy: Mexicostylus squamatus Verhoeff, 1933 non Trichorhina squamata Verhoeff, 1926

Literature: Verhoeff (1926); Verhoeff (1933); VAN NAME (1936); MulaiK (1960)

Distribution: Mexico, Chiapas

Remark: As far as the generic placement and the separate status of "Mexicostylus" squamatus Verhoeff, 1933 and Trichorhina squamata Verhoeff, 1926 are proved, the former is a junior homonym of the latter and must obtain a new specific name.

Trichorhina squamapleotelsona Schultz, 1984

Literature: SCHULTZ (1984c)

Distribution: Belize

Trichorhina thermophila (Dollfus, 1896)

Literature: VAN NAME (1936)

Distribution: Ecuador; Haiti; Jamaica

Trichorhina tomentosa (Budde-Lund, 1893)

Synonymy: Alloniscus tomentosus Budde-Lund, 1893

Literature: Dollfus (1896c); VAN NAME (1936); VANDEL (1963); LEMOS DE CASTRO (1967); VANDEl (1973); VANDEl (1977); Araujo \& Buckup (1996a); Souza-Kury (1997a)

Distribution: Brazil, Pará, Rio de Janeiro, Rio Grande do Sul; Ecuador; Venezuela; Nicaragua; St. Helena; introduced to Europe, Cuba (?)

Trichorhina vandeli Rioja, 1955

Literature: RioJa (1955b); MULAIK (1960); LEMOS DE CASTRO (1964)

Distribution: Mexico, Chiapas

Trichorhina xoltumae Mulaik, 1960

Literature: MULAIK (1960)

Distribution: Mexico, Veracruz

Trichorhina zimpanensis Mulaik, 1960

Literature: MULAIK (1960)

Distribution: Mexico, Hildago

Revta bras. Zool. 16 (1): 1 - 72, 1999 


\section{Balloniscidae Vandel, 1963 \\ Balloniscus Budde-Lund, 1885}

Ballonsicus brevicornis Budde-Lund, 1885

Literature: VAN NAME (1936)

Distribution: USA, Mississippi

Remark: Like Balloniscus nigricans Budde-Lund, 1885, this is a doubtful species occurring far away from the centre of distribution in eastern South America.

Balloniscus glaber Araujo \& Zardo, 1995

Literature: ARAUJO \& ZARDO (1995)

Distribution: Brazil, Rio Grande do Sul

Balloniscus insularum-infra-ventum Vandel, 1952

Literature: VANDEL (1952b); LEMOS DE CASTRO (1976); VANDEL (1981)

Distribution: Islands-under-the-Wind; Venezuela

Balloniscus maculatus Budde-Lund, 1885

Literature: VAN NAME (1936); LEMOS DE CASTRO (1976)

Distribution: Argentinia

Balloniscus nigricans Budde-Lund 1885

Literature: VAN NAME (1936)

Distribution: USA, Mississippi

Remark: cf. Balloniscus brevicornis Budde-Lund, 1885

Balloniscus paraguayanus (van Name, 1936)

Synonymy: Philoscia paraguayana van Name, 1936

Literature: VAn NAME (1936); Lemos de CASTRo (1958a); VANDEL (1963); Schultz (1995);

Distribution: Paraguay

Balloniscus sellowi (Brandt, 1833)

Synonymy: Philoscia sellowi Brandt, 1833

Philoscia paulensis Moreira, 1927

Balloniscus tracheofer Verhoeff, 1941

Philoscia argentina Giambiagi de Calabrese, 1939

Plataoniscus argentinus (Giambiagi de Calabrese, 1939)

Alloniscus argentinus (Dollfus, 1894)

Pardioniscus argentimus (Dollfus, 1894)

Literature: Dollfus (1894); VAN NAME (1936); Giambiagi de CALABReSE (1939); VAN NAME (1940); VerholFF (1941); Lemos de CASTRO (1958a); ARCANGEll (1958); VANDEl (1963); Reca (1970); Lemos de Castro (1976); VANDEl (1981); SChultz (1995); ARAujo et al. (1996)

Distribution: eastern South America

\section{Plataoniscus Vandel, 1963}

Plataoniscus borellii (Dollfus, 1897)

Synonymy: Alloniscus borellii Dollfus, 1897

Literature: VAN NAME (1936); ARCANGel (1958); VANDEL (1963)

Distribution: Argentinia, W Bolivia

Plataoniscus griseus (Dollfus, 1897)

Synonymy: Alloniscus griseus Dollfus, 1897 
Literature: VAN NAME (1936); ARCANGELI (1958); VANDEL (1963)

Distribution: Argentinia

\section{Rhyscotidae Budde-Lund, 1904 \\ Rhyscotoides Arcangeli, 1947}

Rhyscotoides ciferrii (Arcangeli, 1930)

Synonymy: Rhyscotus ciferrii Arcangeli, 1930

Literature: VAN NAME (1936)

Distribution: St. Domingo, Los Hermanos, West Indies

Rhyscotoides cubensis (Budde-Lund, 1908)

Synonymy: Rhyscotus cubensis Budde-Lund, 1908

Literature: VAN NAME (1936); VANDEL (1981)

Distribution: Cuba

Rhyscotoides laxus (van Name, 1924)

Synonymy: Rhyscotus laxus van Name, 1924

Literature: VAN NAME (1936); Mulaik (1960)

Distribution: Galapagos; Mexico, Colima

Rhyscotoides orthonedae (Budde-Lund, 1908)

Synonymy: Rhyscotus orthonedae Budde-Lund, 1908

Literature: VAN NAME (1936)

Distribution: Ecuador, Guayas

Rhyscotoides parallelus (Budde-Lund, 1893)

Synonymy: Rhyscotus parallelus Budde-Lund, 1893

Literature: VAN NAME (1936); VANDEL (1952b); VANDEL (1968); VANDEL (1972a)

Distribution: Venezuela, Caracas; Colombia, Iconozo; Galapagos

Rhyscotus Budde-Lund, 1885

Rhyscotus albidemaculatus (Budde-Lund, 1908)

Literature: VAN NAME (1936); SOUZA-KURY (1997b)

Distribution: Brazil, Rio de Janeiro

Rhyscotus colimensis Mulaik, 1960

Literature: MULAIK (1960)

Distribution: Mexico, Colima

Rhyscotus jacksoni Arcangeli, 1930

Literature: VAN NAME (1936)

Distribution: Santo Domingo, West Indies

Rhyscotus nasutus Budde-Lund, 1908

Literature: VAN NAME (1936)

Distribution: Nicaragua, Realejo

Rhyscotus sphaerocephalus Budde-Lund, 1893

Literature: VAN NAME (1936)

Distribution: Venezuela, Caracas

Rhyscotus texensis (Richardson, 1905)

Literature: VAN NAME (1936); VAN NAME (1940)

Distribution: USA, Texas 
Rhyscotus turgifrons Budde-Lund, 1885

Literature: VAN NAME (1936); MUCHMORE (1993)

Distribution: Virgin Islands, St. Jean

Porcellionidae Brandt \& Ratzeburg, 1831

The family Porcellionidae only comprises species of the Western Palaearctic and some genera of the Aethiopis. A revision of the species described to be native to America will lead to a disposition to other genera and families, or will even prove synonymy with one of the cosmopolitic species.

\section{Agabiformius Verhoeff, 1908}

Agabiformius lentus (Budde-Lund, 1885) \#

Synonymy: Leptotrichus granulatus Richardson, 1902

Leptotrichus panzeri (Audouin, 1826) partim

Porcellionides davisi Mulaik, 1960

Porcellionides hildaguensis Mulaik, 1960

Porcellio gertschi van Name, 1942

Literature: VAN NAME (1936); VAN NAME (1942); MULAIK (1960); SCHUlTZ (1965b); Lemos de CASTro (1971); SChultz (1972b); SCHultz (1984b); MuchMore (1993)

Distribution: southeastern USA, Virgin Islands; Haiti; Bermuda; Mexico; Venezuela; Brazil; Senegal; North Africa

Native distribution: Southern Europe

Agabiformius modestus (Budde-Lund, 1885)

Synonymy: Lyprobius modestus Budde-Lund, 1885

Literature: VAN NAME (1936)

Distribution: Central America (?); Argentinia (?)

Agabifromius pusillus (Budde-Lund, 1885)

Synonymy: Lyprobius pusillus Budde-Lund, 1885

Literature: VAN NAME (1936)

Distribution: USA, California

Leptotrichus Budde-Lund, 1885

Leptotrichus panzeri (Audouin, 1826) \#

Literature: SCHULTZ (1972b); VANDEL (1977)

Distribution: Bermuda (?); St. Helena

Native distribution: Atlantic Archipelagoes, Mediterranean area

\section{Porcellio Latreille, 1804}

\section{Porcellio dilatatus Brandt \& Ratzeburg, 1833 \#}

Synonymy: Porcellio spinicornis occidentalis Miller (1936)

Literature: VAN NAME (1940); HATCh (1947); PALmÉN (1951); Lemos de CASTro (1971); GarthWAite et al. (1985); GarthWAITE \& LaWSON (1992); Araujo et al. (1996)

Distribution: Canada, Newfoundland; USA, Washington, Arizona, California; Brazil, Minas Gerais to Rio Grande do Sul

Native distribution: Western Europe

Porcellio granarus Nicolet, 1849

Literature: VAN NAME (1936)

Distribution: Chile 


\section{Porcellio laevis Latreille, 1804 \#}

Literature: Miers (1877); DOLlFus (1896c); DOLlFus (1897a); CREASER (1936); VAN Name (1936); Van Name (1940); VerhoefF (1941); Hatch (1947); Camargo (1954); ANDERSSON (1960); SCHUltz (1965b); VANDEl (1968); LEMOS DE CASTRO (1971); VAndel (1977); Garthwaite et al. (1985); Jass \& Klausmeier (1990); GARTHWAITE \& LAWSON (1992); ARAUJO et al. (1996)

Distribution: in almost all habitats influenced by man in both North and South America; St. Helena

Native distribution: Southern Europe

Porcellio lamellatus Uljanin, 1875 \#

Synonymy: Porcellio quadrifrons Giambiagi de Calabrese, 1939

Literature: GIAmbiagi de CAlabrese (1939); VAn NAmE (1942); ReCa (1972); SCHUltZ (1972b); VANDEL (1977)

Distribution: Bermuda; Argentinia, Buenos Aires; St. Helena

Native distribution: Southern Europe

Porcellio liliputanus Nicolet, 1849

Literature: VAN NAME (1936)

Distribution: Chile

Porcellio marginalis Mulaik, 1960

Literature: MULAIK (1960)

Distribution: Mexico, Veracruz

Porcellio pubescens Dollfus, 1896

Literature: Dollfus (1896c); VAN NAME (1936); VAN NAME (1942)

Distribution: Venezuela, Petare, Colonie Tovar

Porcellio ragusae (Dollfus, 1896) \#

Literature: VAN NAME (1940)

Distribution: USA, Texas

Native distribution: Southern Europe

Porcellio scaber Latreille, 1804 \#

Synonymy: Porcellio cayennensis Miers, 1877

Porcellio gemmulatus Dana, 1853

Literature: Dollfus (1897a); VAN NAME (1936); Giambiagi de Calabrese (1939); Van Name (1940); Hatch (1947); PAlmén (1951); VerhoefF (1951); Causey (1952), Causey (1953), Strouhal (1961); Vandel (1977); Garthwaite et al. (1985); Garthwaite (1988); Jass \& Klausmeier (1990); Snider (1991); GarthWaiTE \& LAWSON (1992); ARAujo et al. (1996); JASS \& KLAUSMEIER (1996)

Distribution: Canada, Newfoundland; eastern USA; Mexico; West Indies; Argentinia, Buenos Aires; Brazil, Rio Grande do Sul; Chile, Juan Fernandez Islands; St. Helena Native distribution: Western Europe

Porcellio scabrisculus Mulaik, 1960

Literature: MULAIK (1960)

Distribution: Mexico

\section{Porcellio spinicornis Say, 1818 \#}

Literature: VAn NAme (1936); Hatch (1947); CAusey (1952); Jass \& Klausmeier (1990); SNIDER (1991); JASS \& KLAUSMEIER (1996)

Distribution: southeastern Canada; USA, Michigan, Arkansas, Wisconsin; Great Lakes 
region

Native distribution: Southern Europe

Porcellionides Miers, 1877

Porcellionides advena (Stuxberg, 1872)

Literature: VAN NAME (1936)

Distribution: Brazil, Minas Gerais

Porcellionides bermudezi Boone, 1934

Literature: Boone (1934); VAN NAME (1936); VANDEL (1981)

Distribution: Cuba, Rincón de Genuelo

Porcellionides brunneus (Brandt, 1833)

Literature: VAN NAME (1936)

Distribution: species of questionable distribution

Porcellionides floria Garthwaite \& Sassaman (1985)

Literature: GARTHWAite \& SASSAMAN (1985); GARTHWAITE \& LAWSON (1992)

Distribution: southern USA; Mexico, Yucatan;

Porcellionides fuegensis (Dana, 1853)

Literature: VAN NAME (1936)

Distribution: Chile, Tierra del Fuego

Porcellionides habanensis van Name, 1936

Literature: VAN NAME (1936); RIOJA (1956)

Distribution: Cuba, La Habana

Porcellionides minutissimus (Boone, 1918)

Literature: VAN NAME (1936)

Distribution: Bahamas

Porcellionides pruinosus (Brandt, 1833) \#

Synonymy: Porcellionides flavovittata Miers, 1877

Porcellionides jelkinsi Miers, 1877

Literature: Miers (1877); ARCANGEli (1930); VAN NAME (1936); VERHOEFF (1941); PAulian de Félice (1944); Hatch, (1947); CAusey (1952); CAUSey (1953); CamarGO (1954); ANdersson (1960); SChultz (1965b); Lemos de CASTro (1967); SCHULTZ (1975); VANDEL (1977); JASS \& KLAUSMEIER (1990); TAITI \& FERRARA (1991a); MuChmore (1993); Araujo et al. (1996); JASS \& KlAuSMEIER (1996)

Distribution: almost in all anthropogenous habitats in the Americas; cosmopolitian

Native distribution: mediterranean area

Porcellionides saussurei (Dollfus, 1896)

Literature: DollFus (1896a); VAN NAME(1936); VAN NAME (1940); VAN NAME (1942); Mulaik (1960)

Distribution: Mexico

Porcellionides schwencki (Moreira, 1931)

Literature: Giambiagi de CALABRESE (1939); VAN NAME (1942)

Distribution: Argentinia, Buenos Aires; Brazil, São Paulo

Porcellionides sexfasciatus (Koch, 1847) \#

Literature: VAn NAme (1936); ZArdo \& Loyola E Silva (1988); Araujo et al. (1996)

Distribution: Bermuda; Brazil, Rio Grande do Sul

Native distribution: Mediterranean area 
Porcellionides virgatus (Budde-Lund, 1885)

Synonymy: Porcellio virgatus Schultz, 1975

Porcellionides mulaiki van Name, 1936

Literature: VAN NAME (1936); MULAIK (1960); SchUltZ (1975); SChUltZ (1977a); GARTHWAITE et al. (1985)

Distribution: southeastern USA; Mexico, Tamaulipas, Nayarit

Proporcellio Verhoeff, 1907

Proporcellio quadriseriatus Verhoeff, 1907 \#

Literature: VAN NAME (1936)

Distribution: USA, Texas

Native distribution: Mediterranean area

Cylisticidae Vandel, 1963

Cylisticus Schnitzler, 1853

Cylisticus convexus (de Geer, 1778) \#

Literature: VAN NAME (1936); VAN NAME (1940); HATCh (1947); CAUSEY (1953); MULAIK (1960); SCHULTZ (1965b); VANDEL (1977); JASS \& KLAUSMEIER (1990); SNider (1991); JASS \& KlaUSMEIER (1996)

Distribution: Canada, Newfoundland; eastern USA; Mexico, Tixtla; Argentinia; St. Helena

Native distribution: Europe

Cylisticus esterelanus Verhoeff, 1917 \#

Literature: VANDEL (1973)

Distribution: Cuba

Native distribution: Southwestern Europe

Trachelipodidae Strouhal, 1953

Agnara Budde-Lund, 1908

Agnara madagascariensis Budde-Lund, 1908

Literature: TAITI \& FERRARA (1991a)

Distribution: Ascension; Sahel, Madagascar

Nagurus Holthuis, 1949

Nagurus cristatus (Dollfus, 1899) \#

Literature: ArCangel (1930); Van Name (1936); Paulian de Félice (1944); Mulaik (1960); Lemos de CASTRO (1967); Lemos DE CASTRO (1971); Vilela et al. (1971); VANDEL (1973); ARAUJO \& BUCKUP (1996b)

Distribution: pantropical, Central America; northeastern South America; Cuba; Brazil

Native distribution: probably Southeast Asia

Nagurus cubanocolus Vandel, 1981

Literature: VANDEL (1981)

Distribution: Cuba

Nagurus nanus (Budde-Lund, 1908) \#

Literature: VANDEL (1952b); DE ARAUJO \& BUCKUP (1996b)

Distribution: Venezuela; Brazil, Santa Catarina

Native distribution: Southeast Asia

Revta bras. Zool. 16 (1): 1 - 72, 1999 


\section{Pagana Budde-Lund, 1908}

Pagana dimorpha (Dollfus, 1895)

Literature: FERRARA \& TAITI (1981)

Distribution: Madagascar, Seychelles; Ascension

Trachelipus Budde-Lund, 1908

Trachelipus rathkei (Brandt, 1833) \#

Literature: VAN NAME (1936); HATCH (1947); PALMÉN (1951); CAUSEY (1952), CAUSEY (1953); Lemos de CASTRO (1971); SChultz (1975); JasS \& Klausmeier (1990); SNider (1991); JASS \& KlaUSMeIER (1996)

Distribution: Canada, Newfoundland; USA, Arkansas, Michigan, Great Lakes region southwards to Maryland; Brazil, Rio de Janeiro

Native distribution: Europe

Trachelipus richardsonae Mulaik, 1960

Literature: MULAIK (1960)

Distribution: Mexico, Veracruz

Bisilvestriidae Arcangeli, 1929

The family has been established for the monotypic genus Bisilvestria ARCANGELI, 1929 from Cuba. It might be close to the Scleropactidae Verhoeff, 1938, but there are only few data available.

\section{Bisilvestria Arcangeli, 1929}

Bislivestria marrassinii Arcangeli, 1929

Literature: VAN NAME (1936); VANDEL (1973)

Distribution: Cuba, El Cobre

\section{Scleropactidae Verhoeff, 1938}

Most recently a study on the monophyly and extent of the Scleropactidae has been made (FERRARA et al. 1995). Formerly considered to be mainly of neotropical distribution, it now comprises the subfamily Toradjiinae with a oriental distribution and two genera from Southeast Europe (SCHMALFuSS 1995).

\section{Amazoniscus Lemos de Castro, 1967}

Amazoniscus arlei Lemos de Castro, 1967

Literature: Lemos de CAstro (1967); Lemos de CAstro (1969); MAnicAstri (1991)

Distribution: Brazil, Pará

\section{Chileoniscus Taiti, Ferrara \& Schmalfuss, 1986}

Chileoniscus marmoratus Taiti, Ferrara \& Schmalfuss, 1986

Literature: TAITI, FERRARA \& SCIIMAlFusS (1986)

Distribution: Chile, Santiago

Circoniscus Pearse, 1917

Circoniscus amazonicus Lima, 1996

Literatur: LiMA (1996a)

Distribution: Brazil, Amazonas

Circoniscus apeuensis (Lemos de Castro, 1967)

Synonymy: Parsphaeroniscus apeuensis Lemos de Castro, 1967

Literature: LEMOS DE CASTRO (1967); LEMOS DE CASTRO (1970b); SCHMALFuSS (1980b)

Distribution: Brazil, Pará 
Circoniscus bezzi Arcangeli, 1931

Literature: ARCANGELI (1931); VAN NAME (1936); VILELA et al. (1971); SouZA \& LEMOS de CASTRo (1991); SChUltz (1995)

Distribution: Brazil, Pará, Minas Gerais; Paraguay, Canendiyu

Remark: SchulTz (1995) lumps Circoniscus gracilidens Souza \& Lemos de Castro, 1991, C. incisus Souza \& Lemos de Castro, 1991 and C. pallidus Arcangeli, 1936 with this species.

Circoniscus gaigei Pearse, 1915

Synonymy: Parsphaeroniscus ornatus Verhoeff, 1941

Literature: PeARSE (1915); VAN NAME (1936); Verhoeff (1941a); PAULIAN DE FÉLiCE (1944); ANDERSSON (1960); LEMOS DE CASTRO (1967); SCHMALFuSS (1980b); SOUZA \& LEMOS DE CASTRO (1991); SCHULTZ (1995)

Distribution: Colombia, Santa Marta; Brazil, Amazon region; French Guyana; Guyana; Peru, Iquitos

Remark: SCHULTZ (1995) lumps Circoniscus hamatus van Name, 1936, C. intermedius Souza \& Lemos de Castro, 1991 and Paracubaris spinosus Collinge, 1918 with this species.

Circoniscus gracilidens Souza \& Lemos de Castro, 1991

Literature: SOUZA \& LEMOS DE CASTRO (1991); SCHULTZ (1995)

Distribution: Brazil, Rio de Janeiro

Circoniscus hamatus van Name, 1936

Literature: VAn Name (1936); Paulian de FÉlice (1944); Souza \& Lemos de CASTRo (1991)

Distribution: Guiana, Kamakusa; French Guiana

Circoniscus incisus Souza \& Lemos de Castro, 1991

Literature: Souza \& LEMOS DE CASTRO (1991); SCHULTZ (1995)

Distribution: Brazil, Rio de Janeiro

Circoniscus intermedius Souza \& Lemos de Castro, 1991

Literature: Souza \& LEMOS DE CASTRO (1991); SCHULTZ (1995)

Distribution: Brazil, Mato Grosso

Circoniscus pallidus Arcangeli, 1936

Literature: ARCANGELI (1936); SOUZA \& LEMOS DE CASTRO (1991); SCHULTZ (1995)

Distribution: Brazil, São Paulo

Circoniscus spinosus (Collinge, 1918)

Synonymy: Paracubaris spinosus Collinge, 1918

Synarmadillo spinosus Arcangeli, 1927

Literature: ARCangeli (1927); VAN Name (1936); Schmalfuss (1980b); Schultz (1995)

Distribution: Guyana, Mazakuvi

Colomboniscus Vandel, 1972

Colomboniscus regressus Vandel, 1972

Literature: VANDEL (1972a)

Distribution: Colombia, Montserrate, Tibatita

Colomboscia Vandel, 1972

Colomboscia bituberculata Taiti et al., 1995

Literature: TAITI et al. (1995)

Distribution: Colombia, Santa Marta

Revta bras. Zool. 16 (1): 1 - 72, 1999 
Colomboscia cordillerae Vandel, 1972

Literature: VANDEL (1972a); TAITI et al. (1995)

Distribution: Colombia, Caquet, Chisaqua

Colomboscia species Taiti et al., 1995

Literature: TAITI et al. (1995)

Distribution: Colombia, Chisací

Microspaeroniscus Lemos de Castro, 1984

Microsphaeroniscus bicolor Lemos de Castro, 1984

Literature: LEMOS DE CASTRO (1984b)

Distribution: Brazil, São Paulo

Microsphaeroniscus costatus Lemos de Castro, 1984

Literature: LEMOS DE CASTRO (1984b)

Distribution: Brazil, Rio de Janeiro

Microsphaeroniscus pallidus Lemos de Castro, 1984

Literature: LEMOS DE CASTRO (1984b)

Distribution: Brazil, Rio de Janeiro

Microsphaeroniscus squamatus Lemos de Castro, 1984

Literature: LEMOS DE CASTRO (1984b)

Distribution: Brazil, Rio de Janeiro

Microsphaeroniscus violaceus Lemos de Castro, 1984

Literature: LEMOS DE CASTRO (1984b)

Distribution: Brazil, São Paulo

Neosanfilippia Brian, 1957

Neosanfilippia venezuelana Brian, 1957

Literature: BRIAN (1957); SCHULTZ (1981); MANICASTRI (1991)

Distribution: Venezuela, Falcón

Neosanfilippia zoiai Manicastri, 1991

Literature: Manicastri (1991)

Distribution: Ecuador, Esmeraldas

Pittieroniscus Paoletti, 1989

Remark: A species of Scleropactidae is mentioned in PAOLETTI (1989) from Venezuela. Since no description of neither the genus nor a species has been published, this genus has to be treated as a nomen nudum.

\section{Protosphaeroniscus Schmalfuss, 1980}

Protosphaeroniscus tertiarius Schmalfuss, 1980

Literature: SCHMALFUSS (1980b)

Distribution: fossil from Haiti

Richardsoniscus Vandel, 1963

Richardsoniscus portoricensis (Richardson, 1901)

Synonymy: Sphaeroniscus portoricensis Richardson, 1901

Literature: VAN NAME (1936); VANDEL (1963); SCHMALFusS (1980b)

Distribution: Puerto Rico, El Yunque; Guyana 


\section{Scleropactes Budde-Lund, 1885}

Scleropactes andinus Vandel, 1972

Literature: VANDEL (1972a)

Distribution: Colombia, Bogotá, Resina

Scleropactes botosaneanui Vandel, 1973

Literature: VANDEL (1972b); VANDEL (1973)

Distribution: Cuba, Matanzas

Scleropactes cavifrons Jackson, 1928

Literature: VAN NAME (1936)

Distribution: America, doubtful record

Scleropactes concinnus Budde-Lund, 1885

Literature: VAN NAME (1936); SCHMALFuss (1980b)

Distribution: Equador, Tambillo

Scleropactes columbiensis (Pearse, 1915)

Synonymy: Sphaeroniscus columbiensis Pearse, 1915

Parsphaeroniscus columbiensis Vandel, 1963

Literature: VAN NAME (1936); SCHULTZ (1970d); SCHMALFuss (1986)

Distribution: Colombia, Sta. Marta

Scleropactes estherae Arcangeli, 1930

Literature: ARCANGELI (1930); VAN NAME (1936); SCHMALFuSS (1980b)

Distribution: Costa Rica, La Palina

Remark: doubtful member of this genus (SCHMALFUSS 1980b)

Scleropactes gaigei (Pearse, 1917)

Synonymy: Sphaeroniscus gaigei Pearse, 1917

Literature: PEARSE (1917); VAN NAME (1936); SChultz (1970d); SCHMAlFuss (1986)

Distribution: Colombia, Sta. Marta

Scleropactes granulatus (Richardson, 1901)

Synonymy: Synuropus granulatus Richardson, 1901

Literature: VAN NAME (1936); SCHULTZ (1970d)

Distribution: Puerto Rico, El Yunque

Scleropactes incisus Budde-Lund, 1885

Literature: VAN NAME (1936)

Distribution: Peru

Scleropactes pilosus Vandel, 1968

Literature: VANDEL (1968)

Distribution: Colombia, Mosquera; Ecuador

Scleropactes talamancensis Leistikow, 1997

Literature: LEISTIKOW (1997a)

Distribution: Costa Rica, Cordillera de Talamanca

Scleropactes tatei van Name, 1936

Literature: VAN NAME (1936)

Distribution: Ecuador, Naupén

Scleropactes tristani Arcangeli, 1930

Literature: ARCANGELI (1930); VANDEL (1972b); SCHMALFUSS (1980b) 
Distribution: Costa Rica; Puerto Rico

Remark: doubtful member of this genus (SchmaLFuss 1980b)

Scleropactes zeteki van Name, 1926

Literature: VAN NAME (1926); VAN NAME (1936)

Distribution: Panama

Sphaerobathytropa Verhoeff, 1901

Sphaerobathyropa antarctica Vandel, 1963

Literature: VANDEL (1963); SchMALFUSS (1980b)

Distribution: Chile, Neuquen, Rio Negro

Sphaeroniscus Gerstäcker, 1854

Sphaeroniscus bonitanus van Name, 1942

Literature: VAN NAME (1942)

Distribution: Venezuela, Palo Bonito

Sphaeroniscus flavomaculatus Gerstäcker, 1854

Literature: VAN NAME (1936); VANDEL (1972a)

Distribution: Colombia, Capote

Sphaeroniscus frontalis Richardson, 1912

Literature: VAN NAME (1936)

Distribution: Colombia, Viota

Sphaeroniscus gerstaeckeri Vandel, 1968

Literature: VANDEL (1968)

Distribution: Ecuador, Oriente

Sphaeroniscus granulatus Dollfus, 1896

Literature: DolLFus (1896c); VAN NAME (1936)

Distribution: Venezuela, Victoria

Sphaeroniscus guianensis van Name, 1936

Literature: VAN NAME (1936)

Distribution: Guyana

Sphaeroniscus peruvianus (Budde-Lund, 1885)

Literature: VAN NAME (1936)

Distribution: Peru

Sphaeroniscus pilosus Vandel, 1972

Literature: VANDEL (1972a)

Distribution: Colombia, Montserrate

Sphaeroniscus tukeitanus van Name, 1936

Literature: VAN NAME (1936)

Distribution: Guyana, Tukeit

Sphaeroniscus senex (Budde-Lund, 1885)

Literature: VAN NAME (1936)

Distribution: Venezuela

Spherarmadillo Richardson, 1907

Spherarmadillo cavernicola Mulaik, 1960

Literature: MULAIK (1960)

Distribution: Mexico, Veracruz, San Luis Potosí 
Spherarmadillo huatuscensis Mulaik, 1960

Literature: MULAIK (1960)

Distribution: Mexico, Veracruz

Spherarmadillo schwarzi Richardson, 1907

Literature: VAN NAME (1936); Schmalfuss (1980b); SchUltZ (1984c)

Distribution: Guatemala, Belize

\section{Eubelidae Budde-Lund, 1899}

This family has been revised for several times in the last years. It has an Aethiopian distribution, encompassing the Arabian Peninsula, too. Some enigmatic species are found in the oriental region, but their membership in Eubelidae is not founded accurately. Wether the American members of Ethelum Budde-Lund, 1899 might be autochthone species or not has to be proved by a revision of this genus ( $c f$. FERRARA \& SCHMALFuSS 1976).

\section{Elumoides Taiti \& Ferrara, 1983}

Elumoides coecus Taiti \& Ferrara, 1991 \# (?)

Literature: TAITI \& FERRARA (1991a)

Distribution: Ascension

Native distribution: Africa (?)

\section{Ethelum Budde-Lund, 1899}

Ethelum americanum (Dollfus, 1896)

Literature: Dollfus (1896b); VAN NAME (1936); LEMOS DE CASTRO (1967)

Distribution: St. Vincent; Guyana; French Guyana; Brazil, Pará

Ethelum modestum (Dollfus, 1896)

Literature: DolLfus (1896b); VAN NAME (1936)

Distribution: St. Vincent

Ethelum reflexum (Dollfus, 1896)

Literature: Dollfus (1896b); VAN NAME (1936)

Distribution: St. Vincent

Ethelum species Kraeplin, 1901

Literature: VAN NAME (1936)

Distribution: Brazil, San Francisco (?)

Periscyphis Gerstäcker, 1873

Periscyphis species Kaeplin, 1901

Literature: VAN NAME (1936)

Distribution: Brazil (doubtful record)

$$
\begin{aligned}
& \text { Pudeoniscidae Lemos de Castro, } 1973 \\
& \text { Brasiloniscus Lemos de Castro, } 1973
\end{aligned}
$$

Brasiloniscus maculatus Lemos de Castro, 1973

Literature: LEMOS DE CASTRO (1973)

Distribution: Brazil, Rio de Janeiro, São Paulo

Brasiloniscus verrucosus Lemos de Castro, 1973

Literature: LEMOS DE CASTRO (1973)

Distribution: Brazil, Rio de Janeiro 


\section{Pudeoniscus Vandel, 1963}

Pudeoniscus birabeni Vandel, 1963

Literature: VANDEL (1963); LEMOS DE CASTRO (1973)

Distribution: Brazil, Rio de Janeiro

Pudeoniscus obscurus Lemos de Castro, 1973

Literature: LEMOS DE CASTRO (1973)

Distribution: Brazil, São Paulo

Armadillidiidae Brandt, 1833

Armadillidium Brandt, 1830

Armadillidium nasatum Budde-Lund, 1885 \#

Literature: VAN NAME (1936); HATCH (1947); CAUSEY (1953); SCHULTZ (1961a); JASS \& KLAUSMEIER (1990); SNider (1991); ARAuJo et al. (1996)

Distribution: northeastern USA, Arkansas; Brazil, Rio Grande do Sul

Native distribution: Southwest Europe

Armadillidium vulgare (Latreille, 1804) \#

Literature: VAn NAME (1936); HATCH (1947); CAUSEy (1952); CAMARgo (1954); Strouhal (1961); SChultz (1965b); Lemos de CASTRo (1971); SChultz (1975); VANDEl (1977): Ferrara \& TAITI (1981); GarthwaIte et al. (1985); Jass \& KLAUSMeier (1990); SNider (1991); GarthWAite \& LAWSON (1992); Araujo et al. (1996)

Distribution: USA, Arkansas, Michigan, Georgia, Great Lakes region, Texas; Brazil, Rio Grande do Sul; Chile, Juan Fernandez Islands; St. Helena; Ascension

Native distribution: Europe

\section{Eluma Budde-Lund, 1885}

Eluma caelata Miers, 1877 (\#)

Literature: MIERS (1877); VAN NAME (1936)

Distribution: Guyana (autochthone?)

Armadillidae Brandt \& Ratzeburg, 1831

Species of the genera Armadillo Duméril, 1816, Cubaris Brandt, 1833 and Venezillo Verhoeff, 1928 have been used freely in the past. There exist distinct characters to separate the three genera from each other, but particularly in the literature from around the turn of this century to the 1930s, there is much confusion on the generic identity of most neotropical Armadillidae. Therefore, a revision of these species is highly desirable.

\section{Bethalus Budde-Lund, 1908}

Bethalus depressus (Dollfus, 1896)

Literature: DolLFus (1896b); VAN NAME (1936)

Distribution: St. Vincent

Bethalus tenuipunctatus (Dollfus, 1896)

Literature: DolLfus (1896b); VAN NAME (1936)

Distribution: West Indies, Mustique Island

\section{Cosmeodillo Vandel, 1973}

Cosmeodillo decoui Vandel, 1973

Literature: VANDEL (1973)

Distribution: Cuba, La Habana 


\section{Cubaris Brandt, 1833}

Cubaris acapulcensis Mulaik, 1960

Literature: MulaIK (1960)

Distribution: Mexico, Guerrero

Cubaris benitensis Mulaik, 1960

Literature: MULAIK (1960)

Distribution: Mexico, Baja California

Cubaris bolivari Mulaik, 1960

Literature: MULAIK (1960)

Distribution: Mexico, Veracruz

Cubaris cinchonae van Name, 1936

Literature: VAN NAME (1936)

Distribution: Jamaica

Cubaris cineraea Brandt, 1833

Literature: VAN NAME (1936)

Distribution: Brazil (doubtful)

Cubaris flavobrunnea (Dollfus, 1896)

Literature: VAN NAME (1936)

Distribution: Panama, Darién

Cubaris granaria (Nicolet, 1849)

Literature: VAN NAME (1936)

Distribution: Chile

Cubaris margaritae Vandel, 1952

Literature: VANDEL (1952a)

Distribution: Venezuela, Isla Margarita

Cubaris minuta Mulaik, 1960

Literature: MULAIK (1960)

Distribution: Mexico, Colima

Cubaris mirandai Rioja, 1954

Literature: RıOJA (1954); MULAIK (1960)

Distribution: Mexico, Veracruz

Cubaris murina Brandt, 1833 \# (?)

Synonymy: Cubaris brunnea Brandt, 1833

Literature: VAN NAME (1936); SCHUltZ (1961b); LeMOS DE CASTRO (1967); VILELA et al. (1971); SChultz (1972b); VANDEL (1973); TAITI \& FERRARA (1991a); Araujo et al. (1996)

Distribution: pantropic; Cuba; USA, Florida; Brazil, Pará, Mato Grosso, Santa Catarina; Ascension

Diploexochus Brandt, 1833

Diploexochus echinatus Brandt, 1833

Literature: VAN NAME (1936); ARCANGELI (1956); Lemos dE CASTRO (1967)

Distribution: Guyana; Trinidad; Brazil, Pará

Globarmadillo Richardson, 1910

Globarmadillo armatus Richardson, 1910

Synonymy: Synarmadillo armatus Arcangeli, 1927 
Literature: ArCangeli (1927); VAn NAME (1936); Schultz (1970a); Argano \& MANICASTRI (1979)

Distribution: Guatemala, Tres Aguas

\section{Laureola Barnard, 1960}

Laureola atlantica Vandel, 1977

Literature: VANDEL (1977)

Distribution: St. Helena

\section{Pseudodiploexochus Arcangeli, 1934}

Remark: FERRARA \& TAITI (1978) place the species of the genus Reductoniscus Kesselyak, 1930 from St. Helena in the genus Pseudodiploexochus Arcangeli, 1934.

Pseudodiploexochus gibbus (Lemos de Castro, 1972)

Synonymy: Reductoniscus gibbus Lemos de Castro, 1972

Literature: LEMOS DE CASTRO (1972), FERRARA \& TAITl (1990)

Distribution: Brazil, São Paulo

Pseudodiploexochus insularis (Vandel, 1977)

Synonymy: Reductoniscus insularis Vandel, 1977

Literature: VANDEL (1977); FERRARA \& TAITI (1978); FERRARA \& TAITI (1990)

Distribution: St. Helena

Pseudodiploexochus leleupi (Vandel, 1977)

Synonymy: Reductoniscus leleupi Vandel, 1977

Literature: VANDEL (1977); FERRARA \& TAITI (1978); FERRARA \& TAITI (1990)

Distribution: St. Helena

Pseudodiploexochus mellissi (Vandel, 1977)

Synonymy: Reductoniscus mellissi Vandel, 1977

Literature: VANDEL (1977); FERRARA \& TAITI (1978); FERRARA \& TAITI (1990)

Distribution: St. Helena

Pseudodiploexochus tabularis (Barnard, 1932) \#

Literature: FERRARA \& TAITI (1981)

Distribution: Ascension

Native distribution: South Africa

Sphaerillo Verhoeff, 1926

Sphaerillo parvus (Budde-Lund 1885) \# (?)

Literature: FERRARA \& TAITI (1981)

Distribution: Ascension, Seychelles; Mauritius; Chagos and Cocos-Keeling Archipelagoes

\section{Synarmadillo Dollfus, 1891}

Synarmadillo clausus (Budde-Lund, 1885)

Synonymy: Armadillo clausus Budde-Lund, 1885

Cubaris clausa van Name, 1936

Venezillo venezuelae van Name, 1942

Literature: DolLfus (1896c); VAN NAME (1936); ARCANGELI (1956); VANDEL (1952b); SCHMALFUSS (1980b)

Distribution: Venezuela, Caracas 
Synarmadillo monocullatus (Dollfus, 1896)

Synonymy: Haplarmadillo monocullatus Dollfus, 1896

Literature: Dollfus (1896b); ARCANGELI (1927); VAN NAME (1936)

Distribution: St. Vincent

Synarmadillo ruthveni (Pearse, 1915)

Synonymy: Coxopodias ruthveni Pearse, 1915

Literature: PEARSE (1915); ARCANGeli (1927); VAN NAME (1936)

Distribution: Colombia, Santa Marta

Synarmadillo tristani Richardson, 1910

Synonymy: Coxopodias tristani Richardson, 1910

Literature: RICHARDSON (1910); ARCANGELI (1927); VAN NAME (1936)

Distribution: Costa Rica, Turrialba

\section{Venezillo Verhoeff, 1928}

Venezillo aguayoi (Boone, 1934)

Synonymy: Cubaris aguayoi Boone, 1934

Literature: VAN NAME (1936); ARCANGELI (1956)

Distribution: Cuba, Camoa

Venezillo apacheus (Mulaik, 1942)

Synonymy: Cubaris apachea Mulaik, 1942

Literature: VAN NAME (1942); ARCANGELI (1956)

Distribution: USA, Texas

Venezillo arizonicus (Mulaik, 1942)

Synonymy: Cubaris arizonicus Mulaik, 1942

Literature: VAN NAME (1942); ARCANGELI (1956)

Distribution: USA, Arizona

Venezillo articulatus Mulaik, 1960

Synonymy: Armadillo (Venezillo) articulatus Mulaik, 1960

Literature: MULAIK (1960)

Distribution: Mexico, Guerrero

Venezillo beebei (van Name, 1924)

Synonymy: Cubaris beebei van Name, 1924

Literature: VAN NAME (1936); ARCANGELI (1956)

Distribution: Galapagos

Venezillo bellavistanus Schultz, 1995

Literature: SCHULTZ (1995)

Distribution: Paraguay, Amambay

Venezillo bolivianus (Dollfus, 1897)

Synonymy: Armadillo bolivianus Dollfus, 1897

Literature: VAN NAME (1936); ARCANGELI (1956); SCHULTZ (1995)

Distribution: Bolivia, Chaco; Paraguay, Amambay

Venezillo boneti Mulaik, 1960

Synonymy: Armadillo (Venezillo) boneti Mulaik, 1960

Literature: MULAIK (1960)

Distribution: Mexico, Guerrero

Revta bras. Zool. 16 (1): 1 - 72, 1999 
Venezillo booneae (van Name, 1936)

Synonymy: Cubaris booneae van Name, 1936

Literature: VAN NAME (1936); ARCANGELI (1956)

Distribution: Jamaica, Moneague

Venezillo brevispinis (Pearse, 1915)

Synonymy: Cubaris brevispinis Pearse, 1915

Literature: Pearse (1915); VAn NAME (1936); ARCANGeli (1956)

Distribution: Colombia, Sta. Marta

Venezillo cacahuampilensis (Bilimek, 1867)

Synonymy: Armadillo cacahuampilensis Bilimek, 1867

Cubaris cacahuampilensis van Name, 1936

Literature: VAN NAME (1936); Rioja (1955b); ARCANGELI (1956); MUlaik (1960)

Distribution: Mexico, Cacahuampila

Venezillo californicus (Budde-Lund, 1885)

Literature: VAN NAME (1936)

Distribution: USA, California

Venezillo chamberlini (Mulaik, 1942)

Synonymy: Cubaris chamberlini Mulaik, 1942

Literature: VAN NAME (1942); ARCANGELI (1956)

Distribution: USA, Texas

Venezillo chiapensis Rioja, 1955

Literature: RioJA (1955b); ARCANGELI (1956); MULAIK (1960)

Distribution: Mexico, Chiapas

Venezillo colomboi (Arcangeli, 1929)

Synonymy: Cubaris colomboi Arcangeli, 1929

Literature: ARCANGELI (1956); VANDEL (1973)

Distribution: Cuba, La Habana

Venezillo congener (Budde-Lund, 1904)

Synonymy: Armadillo congener Budde-Lund, 1904

Cubaris congenera van Name, 1936

Literature: VAn NAME (1936); ARCANGel (1956); ViLela et al. (1971)

Distribution: Brazil, Mato Grosso

Venezillo culebrae (van Name, 1936)

Synonymy: Cubaris culebrae van Name, 1936

Literature: VAN NAME (1936); ARCANGELI (1956); MuChMORE (1993)

Distribution:West Indies, Culebra Island; Virgin Islands;

Venezillo dugesi (Dollfus, 1896)

Synonymy: Armadillo dugesi Dollfus, 1896

Cubaris dugesi van Name, 1936

Literature: Dollfus (1896a); VAN NAME (1936); ARCANGELI (1956); Mulaik (1960)

Distribution: Mexico, Corritos, Morelia, Michoacan, San Luis Potosí

Venezillo dumorum (Dollfus, 1896)

Synonymy: Armadillo dumorum Dollfus, 1896

Cubaris dumorum van Name, 1936

Literature: Dollfus (1896b); VAN NAME (1936), VANDEL (1952b); ARCANGELI (1956)

Distribution: Grenada; Venezuela, Isla Margarita 
Venezillo galapagoensis (Miers, 1877)

Synonymy: Cubaris galapagoensis Miers, 1877

Literature: MIERS (1877); VAN NAME (1936); ARCANGELI (1956)

Distribution: Galapagos

Venezillo gigas (Miers, 1877)

Synonymy: Cubaris gigas Miers, 1877

Literature: Miers (1877); ARCANGEli (1930); VAN NAME (1936); ARCANGELi (1956)

Distribution: Nicaragua; Costa Rica; Colombia, Sta. Marta

Venezillo grenadensis (Budde-Lund, 1893)

Synonymy: Armadillo grenadensis Budde-Lund, 1893

Cubaris grenadensis van Name, 1936

Cubaris ramsdeni Boone, 1934

Literature: Dollfus (1896b); ARCANGELI (1930); VAN NAME (1936); VANDEL (1952b); ARCANGELI (1956); VANDEL (1981)

Distribution: Colombia; Venezuela; Costa Rica, San José; Cuba, Guantanamo

Venezillo hendersoni (Boone, 1934)

Synonymy: Cubaris hendersoni Boone, 1934

Literature: VAN NAME (1936); ARCANGELI (1956)

Distribution: Haiti, Tomazea

Venezillo jamaicensis (Richardson, 1912)

Synonymy: Cubaris jamaicensis Richardson, 1912

Literature: VAN NAME (1936); ARCANGELI (1956)

Distribution: Jamaica

Venezillo Ilamasi Rioja, 1954

Literature: RiOJA (1954); ARCANGELI (1956); MULAIK (1960)

Distribution: Mexico, Puebla

Venezillo longispinis (Richardson, 1912)

Synonymy: Cubaris longispinis Richardson, 1912

Literature: VAN NAME (1936)

Distribution: Panama

Venezillo macrosoma Mulaik, 1960

Synonymy: Armadillo (Venezillo) macrosoma Mulaik, 1960

Literature: MULAIK (1960)

Distribution: Mexico, Baja California

Venezillo mexicanus (Verhoeff, 1933)

Synonymy: Microdillo mexicanus Verhoeff, 1933

Cubaris mexicana van Name, 1936

Literature: VerhoEfF (1933); VAN NAME (1936); ARCANGEli (1956)

Distribution: Mexico, Guerrero

Venezillo microphthalmus (Arcangeli, 1932)

Synonymy: Armadillo (Diploexochus) microphthalmus Arcangelli, 1932

Cubaris microphthalma van Name, 1936

Literature: VAN NAME (1936); ARCANGELI (1956); GARTHWAITE et al. (1985)

Distribution: USA, California 
Venezillo mineri (van Name, 1936)

Synonymy: Cubaris mineri van Name, 1936

Literature: VAN NAME (1936); VANDEL (1952b); ARCANGELI (1956); VANDEL (1963)

Distribution: Guyana, Kamasuka; Venezuela

Venezillo moneaguensis (van Name, 1936)

Synonymy: Cubaris moneaguensis van Name, 1936

Literature: VAN NAME (1936); ARCANGELI (1956)

Distribution: Jamaica, Moneague

Venezillo multipunctatus (Budde-Lund, 1885)

Synonymy: Armadillo multipunctatus Budde-Lund, 1885

Cubaris multipunctata van Name, 1936

Literature: DolLfus (1896c); VAN NAME (1936); VANDEL (1952b); ARCANGELI (1956)

Distribution: Venezuela, Caracas

Venezillo nevadensis Mulaik, 1960

Synonymy: Armadillo (Venezillo) nevadensis Mulaik, 1960

Literature: MuLAIK (1960)

Distribution: Mexico, Jalisco

Venezillo nigrorufus (Dollfus, 1896)

Synonymy: Armadillo nigrorufus Dollfus, 1896

Cubaris nigrorufa van Name, 1936

Literature: Dollfus (1896c); VAN NAme (1936); VANDEL (1952b); ARCANGELI (1956)

Distribution: Venezuela, Victoria

Venezillo oaxacanus (van Name, 1936)

Synonymy: Cubaris oaxacana van Name, 1936

Literature: VAN NAME (1936); ARCANGELI (1956); Mulaik (1960)

Distribution: Mexico, Guerrero, Oaxaca

Venezillo orosioi Mulaik, 1960

Synonymy: Armadillo (Venezillo) orosioi Mulaik, 1960

Literature: MULAIK (1960)

Distribution: Mexico, Guerrero, Nuevo León

Venezillo parvus (Budde-Lund, 1885)

Synonymy: Venezillo evergladensis Schultz, 1963

Literature: Schultz (1963d); Schultz (1972a); Schultz (1975); Schultz (1977a); KeEney (1991); TAITI \& Ferrara (1991b)

Distribution: USA, Georgia, Florida, Georgia, Ohio, Hawaii

Venezillo perlatus (Dollfus, 1896)

Synonymy: Armadillo perlatus Dollfus, 1896

Cubaris perlatus van Name, 1936

Literature: DollFus (1896b); VAN NAME (1936); ARCANGELI (1956)

Distribution: St. Vincent (or Grenada ?)

Venezillo pisum (Budde-Lund, 1885)

Synonymy: Armadillo pisum Budde-Lund, 1885

Cubaris pisum van Name, 1936

Literature: VAN NAME (1936); ARCANGELI (1956); SCHUltZ (1972a)

Distribution: USA, Florida 
Venezillo phylax (van Name, 1936)

Synonymy: Cubaris phylax van Name, 1936

Literature: VAN NAME (1936); ARCANGELI (1956)

Distribution: Santo Domingo, Cabo Macao

Venezillo pleogoniphorus (Rioja, 1951)

Synonymy: Cubaris pleogoniphora Rioja, 1951

Literature: RioJA (1951a, 1955b); ARCANGELI (1956); MUlaIK (1960)

Distribution: Mexico, San Luis Potosí

Venezillo pumilus (Budde-Lund, 1893)

Synonymy: Armadillo pumilus Budde-Lund, 1893

Cubaris pumila van Name, 1936

Literature: VAN NAME (1936); VANDEL (1952b); ARCANGELI (1956)

Distribution: Venezuela, Las Trincheras, Caracas

Venezillo rubropunctatus (Budde-Lund, 1893)

Synonymy: Armadillo rubropunctatus Budde-Lund, 1893

Cubaris rubropunctata van Name, 1936

Literature: VAN NAME (1936); VANDEL (1952b); ARCANGELI (1956)

Distribution: Venezuela, Las Trincheras, Caracas

Venezillo sanchezi (Boone, 1934)

Synonymy: Cubaris sanchezi Boone, 1934

Literature: VAN NAME (1936); ARCANGELI (1956)

Distribution: Cuba, Vedado

Venezillo scaberrimus (Dollfus, 1896)

Synonymy: Armadillo scaberrimus Dollfus, 1896

Cubaris scaberrima van Name, 1936

Literature: Dollfus (1896c); VAN NAME (1936); VANDEL (1952b); ARCANGELI (1956)

Distribution: Venezuela, La Guaira

Venezillo schultzei Verhoeff, 1933

Synonymy: Cubaris schultzei van Name, 1936

Literature: VAN NAME (1936); ARCANGEli (1956); Mulaik (1960)

Distribution: Mexico, Chilapa (Guerrero ?)

Venezillo silvarum (Dollfus, 1896)

Synonymy: Armadillo silvarum Dollfus, 1896

Cubaris silvarum van Name, 1936

Literature: Dollfus (1896b); VAN NAME (1936); ARCANGELI (1956)

Distribution: St. Vincent

Venezillo similis (Budde-Lund, 1885)

Synonymy: Armadillo similis Budde-Lund, 1885

Cubaris similis van Name, 1936

Literature: VAN NAME (1936); ARCANGELI (1956)

Distribution: ? Central America

Venezillo soyatlanensis Mulaik, 1960

Synonymy: Armadillo (Venezillo) soyatlanensis Mulaik, 1960

Literature: MULAIK (1960)

Distribution: Mexico, Colima, Tabasco, Jalisco

Revta bras. Zool. 16 (1): 1 - 72, 1999 
Venezillo sylvicola Mulaik, 1960

Synonymy: Armadillo (Venezillo) sylvicola Mulaik, 1960

Literature: MULAIK (1960)

Distribution: Mexico, Colima

Venezillo tanneri (Mulaik \& Mulaik, 1942)

Synonymy: Cubaris tanneri Mulaik \& Mulaik, 1942

Literature: VAN NAME (1936); ARCANGELI (1956); SCHUlTz (1965b)

Distribution: USA, Texas; Mexico

Venezillo truncorum (Budde-Lund, 1893)

Synonymy: Armadillo truncorum Budde-Lund, 1893

Cubaris truncorum van Name, 1936

Literature: Dollfus (1896c); VAN NAME (1936); VANDEL (1952b); ARCANGELI (1956)

Distribution: Venezuela, Caracas, San Estéban, Sta. Lucía

Venezillo tuberosus (Budde-Lund, 1904)

Synonymy: Armadillo tuberosus Budde-Lund, 1904

Cubaris tuberosa van Name, 1936

Literature: VAN NAME (1936)

Distribution: Haiti, Port au Prince

Venezillo venustus (Budde-Lund, 1893)

Synonymy: Armadillo venustus Budde-Lund, 1893

Cubaris venusta van Name, 1936

Literature: DolLFus (1896c); VAN NAME (1936); VANDEL (1952b); ARCANGELI (1956)

Distribution: Venezuela; Trinidad

Venezillo verrucosus (Budde-Lund, 1904)

Synonymy: Armadillo verrucosus Budde-Lund, 1904

Cubaris verrucosa van Name, 1936

Literature: VAN NAME (1936); ARCANGELI (1956)

Distribution: Ecuador, Guayaquil

Venezillo vincentis (Budde-Lund, 1904)

Synonymy: Armadillo vincentis Budde-Lund, 1904

Cubaris vincentis van Name, 1936

Literature: VAN NAME (1936); ARCANGELI (1956)

Distribution: St. Vincent

Venezillo viticola (Dollfus, 1896)

Synonymy: Armadillo viticola Dollfus, 1896

Cubaris viticola van Name, 1936

Literature: DolLFus (1896b); VAN NAME (1936); ARCANGELI (1956)

Distribution: Grenada

Venezillo walkeri (Pearse, 1911)

Synonymy: Cubaris walkeri Pearse, 1911

Literature: VAN NAME (1936); ARCANGELI (1956), MulAiK (1960)

Distribution: Mexico, Veracruz

Venezillo wartoni (van Name, 1936)

Synonymy: Cubaris wartoni van Name, 1936

Literature: VAN NAME (1936); ARCANGELI (1956)

Distribution: Jamaica, Mandeville 
Venezillo wheeleri (van Name, 1936)

Synonymy: Cubaris wheeleri van Name, 1936

Literature: VAN NAME (1936); ARCANGELI (1956)

Distribution: West Indies, Culebra Island

Venezillo zigzag (Dollfus, 1896)

Synonymy: Armadillo zigzag Dollfus, 1896

Cubaris zigzag van Name, 1936

Literature: VAN NAME (1936); ARCANGELI (1956)

Distribution: St. Vincent

Pseudarmadillidae Vandel, 1973

Pseudarmadillo Saussure, 1857

Pseudarmadillo buschki Richardson, 1905

Literature: VAN NAME (1936)

Distribution: Cuba, Caenito

Pseudarmadillo carniculatus Saussure, 1857

Literature: VANDEL (1973)

Distribution: Cuba, Oriente

Pseudarmadillo cristatus Schmalfuss, 1984

Literature: SchmaLFuss (1984)

Distribution: fossil from Haiti

Pseudarmadillo dollfusi Richardson, 1905

Literature: VAN NAME (1936); VANDEL (1973)

Distribution: Bahamas, Andros

Pseudarmadillo gillianus Richardson, 1902

Synonymy: Pseudarmadillo welchi Boone, 1904

Literature: VANDEL (1973), SCHMALFUSS (1984)

Distribution: Cuba

Pseudarmadillo hoplites (Boone, 1934)

Synonymy: Delatorella holpites Boone, 1934

Literature: BOONEae (1934); VAN NAME (1936); VANDEL (1973); SCHMALFuSS (1984)

Distribution: Cuba, Camaguey

Pseudarmadillo tuberculatus Schmalfuss, 1984

Literature: SCHMALFUSS (1984)

Distribution: fossil from Haiti

\section{ADDITIONAL REMARKS}

At least 37 species of Oniscidea were introduced to this region by human activities. Most of these species are anthropophilous and thus mainly can be found in the vicinity of human settlements. Several species of the Nearctic Region seem to be well established in acceptable habitats as is Armadillidium vulgare (Latreille, 1804) in the southeastern parts of the USA (SCHULTZ 1961). Species which most probably are introduced and their presumed origin are summarized in table I.

It also has to be stressed that several species described from South and Central America are of uncertain systematic position and even their validity as full species has to be 
Table I. Oniscidea introduced to the Americas.

\begin{tabular}{|c|c|c|}
\hline Family & Species & Origin \\
\hline Ligiidae & Ligia oceanica & Western Europe \\
\hline \multirow[t]{7}{*}{ Trichoniscidae } & Androniscus dentiger & Central Europe \\
\hline & Haplophthalmus danicus & Central Europe \\
\hline & Hyloniscus riparius & Central Europe \\
\hline & Trichoniscoides sarsi & Central Europe \\
\hline & Trichoniscus provisorius & Southeastern Europe \\
\hline & Trichoniscus pusillus & Western Europe \\
\hline & Trichonsicus pygmaeus & Western Europe \\
\hline Styloniscidae & Clavigeroniscus riqueri & Doubtful \\
\hline Stenoniscidae & Stenoniscus pleonalis & Southern Europe \\
\hline \multirow[t]{2}{*}{ Philosciidae } & Burmonsicus meeusi & Southeast Asia \\
\hline & Philoscia muscorum & Central Europe \\
\hline Halophilosciidae & Halophiloscia couchi & Western Europe \\
\hline On̦iscidae & Oniscus asellus & Central Europe \\
\hline \multirow[t]{4}{*}{ Platyarthridae } & Niamba capensis & West Africa \\
\hline & Niamba squamata & South Africa \\
\hline & Platyarthrus aiasensis & Southern Europe \\
\hline & Platyarthrus hoffmannseggi & Central Europe \\
\hline \multirow[t]{9}{*}{ Porcellionidae } & Agabiformius lentus & Southern Europe \\
\hline & Leptotrichus panzeri & Southern Europe \\
\hline & Porcellio dilatatus & Southwestern Europe \\
\hline & Porcellio laevis & Southern Europe \\
\hline & Porcellio scaber & Western Europe \\
\hline & Porcellio spinicornis & Wetsern Europe \\
\hline & Porcellionides pruinosus & Southern Europe \\
\hline & Porcellionides sexfasciatus & Southern Europe \\
\hline & Proporcellio quadriseriatus & Southern Europe \\
\hline \multirow[t]{5}{*}{ Trachelipodidae } & Agnara madagascariensis & Indopacific region \\
\hline & Nagurus cristatus & Southeast Asia (?) \\
\hline & Nagurus nanus & Southeast Asia \\
\hline & Pagana dimorpha & Indopacific region \\
\hline & Trachelipus rathkei & Central Europe \\
\hline \multirow[t]{2}{*}{ Cylisticidae } & Cylisticus convexus & Central Europe \\
\hline & Cylisticus esterelanus & Southwestern Europe \\
\hline \multirow[t]{2}{*}{ Armadillidiidae } & Armadillidium nasutum & Southwestern Europe \\
\hline & Armadillidium vulgare & Central Europe \\
\hline Armadillidae & Cubaris murina & South Asia (?) \\
\hline
\end{tabular}

doubted. Some of them will prove to be synonymous to the cosmopolitic species of Porcellio Latreille, 1804 or of Porcellionides Miers, 1877. This seems probable since even the type species of the latter genus (Porcellionides jelkinsi Miers, 1877) is a junior synonym of Porcellionides pruinosus (Brandt, 1833), a cosmopolitican species as indicated above (FERRARA \& SCHMALfuss 1983). Some of them, like the so-called members of the genus Philoscia Latreille, 1804, will be placed in other genera. None of the described species show the characters of the genus Philoscia, in so far as it could be interpreted from the poor descriptions and illustrations given by authors like DOLLFus (1893a, b) and VERHOEFF (1933). Relevant characters were not recorded and the ascription to any genus must be random. A good example is the record of "Oniscus" armatus Nicolet, 1849 for the difficulties 
in judging the validity of species. The poor description does not give aid in any taxonomic question. The only statement that can be made is the high probability that this species is erroneous, since the genus Oniscus Linné, 1767 is of West Palaearctic distribution and it might be more possible that the record refers to Oniscus asellus Linné, 1767 as an anthropophilous species. There has to be made huge efforts to clarify the taxonomic questions raised within this work and research has to improve our knowledge on this interesting phylum.

ACKNOWLEDGEMENTS. The authors would like to express their thanks to Dr. H. SCHMALFUSS, Staatliches Museum für Naturkunde, Stuttgart, Germany, for the comments on several incertain taxonomic groups. We are indebted to P.B. de ARAUJO, Universidade Federal do Rio Grande do Sul, Porto Alegre, Brazil, and Dr. F. Ferrara, Università di Firenze, Italy for the provision with some literature of difficult access.

\section{REFERENCES}

Andersson, A. 1960. South American terrestrial isopods in the collection of the Swedish State Museum of Natural History. Arkiv Zool. 12: 537-570.

ARAujo, P.B. DE \& L. BuCKuP 1994a. Two new species of terrestrial Isopoda from southern Brazil. Spixiana 17: 269-274. 129-134.

1994b. Nova especie de Trichorhina do sul do Brasil. Iheringia, Sér. Zool. (77):

. 1996a. Novos registros e uma espécie nova de Trichorhina Budde-Lund (Isopoda; Oniscidea, Platyarthridae) do sul do Brasil. Revta bras. Zool. 13 (3): 799-810.

-1996b. Ocorrência de Nagurus Holthuis, 1949 (Isopoda, Trachelipodidae) no sul do Brasil. Nauplius 4: 161-163.

Araujo, P. B.; L. Buckup \& C. Bond-Buckup. 1996. Isópodos terrestres de Santa Catarina e Rio Grande do Sul. Iheringia, Sér. Zool., (81): 111-138.

Araujo, P. B. \& C.M.L. ZARDo. 1995. Uma nova espécie de Balloniscus do sul do Brasil. Revta bras. Zool. 12 (4): 785-790.

ARCANGELI, A. 1297. Revisione dei generi degli isopodi terrestri. 1.a nota - Sopra alcuni generi di Africa e di America. Atti Soc. ital. Sci. Nat. 66: 126-141

-1930. Contributo alla conoscenza del "Microgenton" di Costa Rica. Boll. Lab. Zool. Agr. Fac. Agraria 25: 1-29.

1931. Circoniscus bezzi, nuova specie di Isopodo terrestre del Brasile. Boll. Zool.

2: $115-122$.

. 1932. Isopodi terrestri di Dominica. Boll. Mus. Zool. Anat. comp. Univ. 42: 1-5.

1936. Un genere e due especie nuovi di Isopodi terrestri del Brasile. Arch. zool. ital. 23: 201-208.

1956. I generi Diploexochus, Venezillo, Pararmadillo. Boll. Ist. Mus. Zool. Univ. 5: 101-142.

-1958. Le especie di isopodi terrestri che furono erroneamente assegnate al genere Alloniscus. Mem. Mus.Civ. Storia Nat. Verona 6: 239-252.

ARgano, R. \& C. ManiCASTRI 1979. A new Akermania from Sri Lanka. Rev. suisse. Zool. 86: 61-68.

Booneat, L.1934. New and rare Cuban and Haitian terrestrial isopods. Bull. Amer. Mus. Nat. Hist. 66: 567-598.

BOWMAN, T.E. 1965. Xilitlonisczs, a new genus for the mexican troglobitic isopod Cordioniscus laevis Rioja (Oniscoidea: Trichoniscidae). Proc. Biol. Soc. Wash. 78: 209-216.

. 1977. Isopod crustaceans (except Anthuridae) collected on the presidental cruise of 1938. Proc. Biol. Soc. Wash. 89: 653-666. 
BRIAN, A. 1957. Descrizione de Neosanfilippia venezuelana n.gen. n.sp. di isopodo terrestre troglobio. Ann. Mus. Civ. Stor. Nat. 69: 352-360.

Camargo, O. R. 1954. Isopodes terrestres do Rio Grande do Sul. Rev. Agronomica 209-211: 122-128.

Causey, D. 1952. The terrestrial isopods of Arkansas. Proc. Arkan. Acad. Sci. 5: 25-30.

. 1953. Additional records of terrestrial isopods from Arkansas. Proc. Arkan. Acad. Sci. 6: 49-50.

Collinge, W.E. 1946. Description of a new species of Ligia from Trinidad (Terrestrial Isopoda). Ann. Mag. Nat. Hist. Ser. 11 13:137-140.

CREASER, E.P. 1936. Crustaceans from Yucatan. Cargenie Inst. Washington 457: 117-132.

Dollfus, A. 1894. Viaggio del dottore Alfredo Borelli nella Repubblica Argentina e nel Paraguay. Boll. Mus. Zool. Anat. comp. Univ. 183: 1-3.

. 1896a. Sur les isopodes terrestres du Mexique. Bull. Soc. Zool. France: 46-49

. 1896b. On West Indian Terrestrial Isopod Crustaceans. Proc. Zool. Soc. London: $388-400$.

$339-345$.

27: 205-212.

. 1897b. Viaggio del dottore Alfredo Borelli nel Chaco Boliviano e nella Republica Argentina. Boll. Mus. Zool. Anat. comp. Univ. 289: 1-4.

EberLey, W. R. 1954. The terrestrial Isopods of Indiana. Proc. Indiana Acad. Sci. 63: 272-277.

FERrARA, F. 1977. Osservazione sistematiche sui generi Exzaes Barnard, 1932 e Hekelus Barnard, 1932 con descrizione di una nuova specie. Rev. Zool. afr. 91: 607-617.

FERrara, F.; C. MEli \& S. TAITI. 1995. Taxonomic revision of the subfamily Toradjiinae (Crustacea: Oniscidea: Scleropactidae). Zool. J. Linnean Soc. 113: 351-459.

Ferrara, F: \& H. Schmalfuss. 1976. Terrestrial isopods from West Africa, part 1: "Eubelidae" Budde-Lund, 1899. Mon. zool. ital. 7: 1-114.

Ferrara, F. \& S. TAiti. 1978. A check-list of terrestrial isopods from Africa. Mon. zool. ital. 12: 89-215.

_. 1981. Terrestrial Isopods from Ascension Island. Mon. zool. ital. 14: 189-198.

\section{3: 1033-1039.}

1989. A new genus and species of terrestrial isopod from Malaysia. J. Nat. Hist.

1990. Two new species of Reductoniscus from New Guinea (Crustacea, Isopoda, Oniscidea). Rev. suisse. Zool. 97: 489-497.

GARCÉs, H. A. 1991. Isopod crustaceans found at Lake Wyman, Boca Raton, Florida. Texas J. Sci. 43: 219-221.

GaRTHWAITE, R.L. 1988. Detonella papillicornis Richardson (Isopoda: Oniscoidea: Scyphacidae) from Bolinas Lagoon, California. Bull. South. Cal. Acad. Sci. 87: 46-47.

Garthwaite, R.L., F.G. Hochberg \& C.S. Sassaman. 1985. The ocurrence and distribution of terrestrial isopods (Oniscoidea) on Santa Cruz Island with preliminar data for other Californian islands. Bull. South Cal. Acad. Sci. 84: 23-27

Garthwatte, R.L. \& R. Lawson. 1992. Oniscidea from the San Francisco Bay area. Proc. Cal. Acad. Sci. 47: 303-328.

GARTHWAite, R.L.; R. LAwson \& S.TAITI. 1992. Morphological and genetical relationships among four species of Armadilloniscus Uljanin, 1875. J. Nat. Hist. 26: 327-338.

GARTIIWAITE R.L. \& C.S. SASSAMAN. 1985. Porcellionides floria, new species, from North America; proviniciality in the cosmopolitan isopod Porcellionides pruinosus (Brandt, 1833). J. Crust. Biol. 5: 539-555.

Garthwaite, R.L. \& S. TAiti. 1989. Platyarthrus aiasensis Legrand in the Americas. Bull. 
South. Cal. Acad. Sci. 88: 42-43.

Giambiagi de Calabrese, D. 1935. Isopodos nuevos para la fauna Argentina. Physis 11: 509.

- 1939. Estudio de los isopodos terrestres argentinos. Physis 17: 633-644.

GRUNER, H.-E. 1955. Die Gattung Benthana. Zool. Jahrb. Syst. 83: 441-451.

HAtCH, M.H. 1947. The Chelifera and Isopoda of Washington and adjacent regions. Univ. Wash. Publ. Biol. 10: 155-274.

HolsingER, J.R.1967. New data on the range of the troglobitic trichoniscid isopod Caucasonethes henrothi. J. Tennessee Acad. Sci. 42: 15.

JACKSON, H.G. 1922. A revision of the isopod genus Ligia (Fabricius). Proc. Linnean Soc.: 683-703.

. 1927. A new subgenus of Ligia, with further observations on the genus. Ann. Mag. Nat. Hist. (9) 14: 129-136.

. 1941. Check-list of terrestrial and fresh water isopods from Oceania. Smithonian Misc. Coll. 99: 1-35.

JASS, J. \& B. Klausmeier 1990. Terrestrial isopod species recorded from the Great Lakes region. Great Lakes Entomol. 23: 165-170

Jass, J. \& B. Klausmeier 1996. Terrestrial isopods (Isopoda: Oniscidea) of Wisconsin. Great Lakes Entomol. 29: 11-20

JoHnson, C. 1986. Parthogenetic reproduction in the Philosciid isopod Ocelloscia floridana. Crustaceana 51: 123-132

JUDD, W. W. 1965. Terrestrial sowbugs in the vicinity of London, Ontario. Canad. Field Nat. 79: 197-202

KeEnEy, G. D. 1990. Some exotical terrestrial isopods from the Columbus Zoo Exploration Center, Powell, Ohio: two new state records. Ohio J. Sci. 90: 133-134

LEISTIKOW, A. 1997a. Terrestrial isopods from Costa Rica, with redescription of Ischioscia variegata (Dollfus, 1896) from Venezuela. Can. J. Zool. 75: 1415-1464

. 1997b. Description of Mirtana costaricensis gen. et sp. n. from Costa Rica (Isopoda: Oniscidea). Stud. neotrop. fauna environm. 32: 118-127.

1998a. Redescriptions of terrestrial Iopoda from Chile and Peru (Crustacea: Isopoda: Oniscidea). Spixiana 21(3): 215-225.

-1998b Considerations about the genus Pentoniscus Richardson, 1913 (Crustacea: Isopoda: Oniscidea) with description of a new species. J. Nat. Hist. 32: 1339-1355.

. 1998c. The genus Pseudophiloscia Budde-Lund, 1904 (Crustacea: Isopoda: Oniscidea) in South America. Mitt. Mus. Nat.kd. Berl., Zool Reihe 74: 233-241.

LEMOS DE CASTRO, A. 1952. Sobre a ocorrência do gênero Tylos Latreille no litoral brasileiro (Isopoda: Tylidae). Bolm. Mus. Nac. (N.S.) 107: 1-7.

- 1953. Fauna do Districto Federal, 8: Sôbre a Ocorrência dos Gêneros "Miktoniscus" e "Cordioniscus" no Rio de Janeiro (Isopoda: Trichoniscidae). An. Acad.

Bras. Cien. 25: 527-534.

1955. Ischioscia amazonica, uma nova espécie de isópode terrestre do Estado do Amazonas (Isopoda: Oniscidae). Rev. Brasil. Biol. 15: 1-8.

Mus. Nov. 1908: 1-10. 85-118.

1958b. Revisão do gênero Benthana Budde-Lund, 1908. Arq. Mus. Nac. 46:

1958c. Benthanosica longicaudata, new genus and species of terrestrial isopod of the family Oniscidae (Isopoda:Oniscoidea). Amer. Mus. Nov. 1884: 1-7

Mus. Nac. (Zool.) 238: 1-7.

1960. Sôbre as espécies americanas de Phalloniscus Budde-Lund. (Isopoda: 
Oniscidae) com descrição de 4 espécies novas. Actas Trab. 1. Congr. Sudam. Zool. 2: 203-211.

1964. Trichorhina heterophthalma, nueva espécie de isopodo terrestre cavernicola de Cuba. Poeyana Ser. A 2: 1-7.

. 1967. Isópodos terrestres da Amazônia Brasileira. Atas Simp. Biota Amaz. 5: 311-336.

1968a. On the systematics of the Genus Littorophiloscia Hatch (Isopoda: Oniscidae). Arquiv. Mus. Nac. 53: 85-98.

1968b. Descrição complementar de Calycuoniscus goeldi (Lemos de Castro) (Isópodes terrestres: Oniscidae: Bathytropinae). Rev. Brasil. Biol. 28: 407-412

- 1969. Descrição complementar de Amazoniscus arlei (Isópodes terrestres: Eubelidae). Bol. Mus. Nac. (Zool.) 269: 1-5.

- 1970a. Considerações sobre o gênero Dubioniscus Vandel com descrição de uma espécie nova. Bol. Mus. Nac. (Zool.) 274: 1-5.

- 1970b. Descrição complementar de Parsphaeroniscus apuensis Lemos de Castro (Isópodes terrestres: Eubelidae). Atas Soc. Biol. Rio de Janeiro 13: 41-42.

- 1970c. Descrição complementar de Phalloniscus singularis Lemos de Castro (Isópodes terrestres: Oniscidae). Atas Soc. Biol. Rio de Janeiro 13: 119-120.

-1970d. Quarto espécies novas de isópodes terrestres do gênero Neotroponiscus Arcangeli do Brasil (Oniscidae: Bathytropinae). Bol. Mus. Nac. (Zool.) 275: 1-15.

. 1970e. Isópodes terrestres do gênero Neotroponiscus Arcangeli (Oniscidae: Bathytropinae). An. Acad. Bras. Cien. 42: 89-95.

$1-14$.

1972. Considerações sôbre o gênero Reductoniscus com descrição de uma espécie nova (Isopoda: Oniscidea). Rev. Brasil. Biol. 32: 347-349.

1973. Pudeoniscidae, fam. nov., com descrição de um gênero novo e três espécies novas de isópodes terrestres do Brasil (Isopoda: Oniscidea). Bol. Mus. Nac. (Zool.) 287: $1-10$.

1976. Considerações sôbre a sinonímia e distribução de Balloniscus sellowi (Brandt, 1833) (Isopoda: Balloniscidae). Rev. Brasil. Biol. 36: 391-396

- 1984a. Uma nova espécie de Prosekia de uma floresta inundável (Igapo) na Amazonia Central. Amazoniana 8: 441-445.

-1984b. Mikrosphaeroniscus, gênero novo de Isópode terrestre volvacional, com descrição de cinco espécies novas (Isopoda: Oniscidea). Bol. Mus. Nac. (Zool.) 308: 1-8 . 1985a. Duas espécies novas brasileiras de Benthana Budde Lund, 1908 (Isopoda: Oniscidea: Philosciidae). Rev. Brasil. Biol. 45: 241-248.

1985b. Considerações sôbre Atlantoscia alceui Ferrara \& Taiti, 1981 (Isopoda: Oniscidea: Philosciidae). Rev. Brasil. Biol. 45: 417-422.

Lemos de CAstro, A. \& L.A. Souza 1986. Três espécies novas de isópodes terrestres do gênero Prosekia Vandel da Amazônia Brasileira (Isopoda: Oniscidea: Philosciidae). Rev. Brasil. Biol. 46: 429-438.

LiMA, I.M.B. 1996a. A new species of Circoniscus Pearse,1917 (Crustacea: Isopoda: Scleropactidae) from the Amazonian Region of Brazil. Amazoniana. 14 (1-2): 91-100.

-1996b. A new species of Prosekia Vandel,1968 (Crustacea: Isopoda: Philosciidae) from Amazonia of Brazil. Amazoniana 14 (1-2): 101-108.

LimA, I.M.B. \& C.S. SEREJo. 1993. A new species of Benthana Budde-Lund from Brazilian caves. Proc. Biol. Soc. Wash. 106: 490-496.

ManiCASTRI, C. 1991. A new species of terrestrial isopods from Equador: Neosanfilippia zoiai spec. nov.. Stud. Neotrop. Fauna Environ. 26: 33-38.

MENZIES, R. 1950. Notes on Californian isopods of the genus Armadilloniscus. Proc. Cal. 
Acad. Sci. 36: 467-481.

Miers, E.J. (1877. On a collection of Crustacea, Decapoda and Isopoda, chiefly from South America. Proc. zool. Soc. London: 653-679.

Muchmore, W.B. 1963. New terrestrial Isopods from the genus Miktoniscus from eastern U.S.. Ohio J. Sci. 64: 51-57.

1970. A new troglobitic trichoniscid isopod of the genus Caucasonethes. J. Tennessee Acad. Sci. 45: 27-28.

1993. List of terrestrial invertebrates of St. John, U.S.Virgin Islands (exclusive Acarina and Insecta), with notes on some records of fresh water species. Caribbean $\mathbf{J}$.

Sci. 29: 30-38.

MulAik, S.B. 1960. Contribución al conocimiento de los isopodos terrestres de Mexico. Rev.

Soc. Mex. Hist. Nat. 21: 79-292.

Palmén, E. 1951. A survey of the Oniscoidea of New Foundland. Ann. Soc. Zool. Bot. Fennica 14: 1-27.

Paoletti, M.G. 1989. Life strategies of Isopods and "soil invertebrates" in Venezuela. Mon. zool. ital. (Monogr.) 4: 435-453.

Paoletti, M.G. \& B.R. StinNer. 1989. Two new terrestrial isopods from coralline cays of Venezuela's caribbean coast. Proc. Entomol. Soc. Wash. 91: 71-80.

PAulian DE FÉlice, L. 1944. Les Oniscoides de la Guyane Francaise. Rev. Franc. Entomol. 10: $142-145$.

PEARSE, A.S. 1915. An account of the Crustacea collected by the Walker Expedition to Santa Marta. Proc. US Nat. Mus. 49: 531-556.

PECK, S.B. 1970. The terrestrial arthropod fauna of Florida caves. Florida Entomol. 53: 203-207.

RECA, A.R. 1970. Oniscoideos argentinos 1: Sobre la posición sistematica de Philoscia argentina Giambiagi, 1939. Physis 29: 423-429.

-1972. Oniscoideos argentinos 2: Tres espécies de isopodos terrestres de la costa maritima bonarense. Physis 31: 405-410.

- 1973. Oniscoideos argentinos 3: aporte al conocimiento de la subfamilia Bathytropinae. Physis, Sección C 32: 93-99.

REDELL, J.R. 1970. A Checklist of the cave fauna of Texas. IV. Additional records of Invertebrata (exclusive of Insecta). Texas J. Sci. 21: 389-415.

Richardson, H. 1910. Terrestrial isopods collected in Costa Rica by J.F. Tristan with description of a new genus and species. Proc. US Nat. Mus. 39: 93-95.

-1913. Terrestrial isopods collected in Costa Rica by Mr. Picardo with descriptions of a new genus and species. Proc. US Nat. Mus. 44: 337-340.

RioJA, E. 1950. Los trichoniscidos cavernicolas de México del género Protrichoniscus y descripción de una nueva specie del mismo. An. Inst. Biol. Méx. 21 (1): 127-146.

—.1951a. Descripción de una nueva espécie del género Cubaris (Isopodos: Cubarido) de la Cueva de los Sabinos (San Luis Potosí). An. Inst. Biol. Méx. 22: 517-524.

- 1951b. Descripción de Protrichoniscus acostai n. sp. de (Crust. isopodo) de Comitan, Chiapas. An. Inst. Biol. Méx. 22 (1): 181-189.

- 1952. Un nuevo género de isópodos trichoniscidos de la Cueva de Ojo Grande, Paraje Neuvo, Córodoba. An. Inst. Biol. Méx. 23: 227-241.

. 1954. Algunas especies de Armadilidios de las cuevas de Mexico (Isopoda). An. Inst. Biol. Méx. 25: 275-288.

1955a. Dos nuevos isopodos cavernicolas de la Sierra Madre Oriental (Reg. de Xilitla), Mexico. An. Inst. Biol. Méx. 26: 447-457.

- 1955b. Observaciones acerca de dos nuevas espécies de isopodos cavernicolas de Chiapas. An. Inst. Biol. Méx. 26: 199-209.

1955c. Trichoniscidae cavernicolas de México. Rev. Soc. Mex. Ent. 1(1-2): 39-62. 
. 1956. Datos sobre algunos isopodos de la isla de Cuba. An. Inst. Biol. Méx. 27: 437-472.

1957. Decripción y estudio de una especie nueva del genero Cylindroniscus (Isopodo trichoniscido) de Yucatan. An. Inst. Biol. Méx. 28: 267-278.

-1964. Descripción y algunos datos morfologicos de Alloniscus thalassophilus spec. nov.. An. Inst. Biol. Méx. 34: 285-300.

SCHMALFUSS, H. 1978. Ligia simoni: A model for the evolution of terrestrial isopods. Stutt. Beitr. Naturk. Ser. A 317: 1-5.

-1980a. A revision of the neotropical genus Ischioscia Verhoeff, with description of four new species (Isopoda, Philosciidae). Stud.Neotrop.Fauna Environ. 15: 125-139

-1980b. Die ersten Landasseln aus Dominikanischem Bernstein mit einer Revision der Familie Sphaeroniscidae (Stuttgarter Bernsteinsammlung: Crustacea: Isopoda: Oniscidea). Stutt. Beitr. Naturk. Ser. B 61: 1-12.

- 1984. Two new species of the terrestrial isopod genus Pseudarmadillo from Dominican amber. Stutt. Beitr. Naturk. Ser. B 102: 1-14.

1986. Die Landisopoden Griechenlands. 8. Beitrag: Kefalloniscus gen. n.. Rev. suisse Zool. 93: 279-289.

- 1995. Die Landisopoden Griechenlands. 16. Beitrag: Xeroporcellio und Kithironiscus gen. n.. Ann. Naturhist. Mus. Wien 978: 139-150.

SChMAlFuss, H. \& F. FERRARA. 1978. Terrestrial isopods of West Africa, part 2: families Tylidae, Ligiidae, Trichoniscidae, Styloniscidae, Rhyscotidae, Halophilosciidae, Philosciidae, Platyarthridae, Trachelipidae, Porcellionidae, Armadillidiidae. Mon. zool. ital. 11: $15-97$

SchotTe, M. \& R.W. HeArd. 1991 Studies on the Crustacea of the Turks and Caicos Islands 2. Gulf Res. Rep. 8: 247-250.

Schultz, G.A. 1961a. Distribution and establishment of a Land Isopod in North America. Syst. Zool. 10: 193-196.

- 1961b. Cubaris murina Brandt, an isopod crustacean new to the United States. Crustaceana 3: 169-170.

.1962. Miktoniscus grayi, a new species of terrestrial isopod crustacean from North Carolina. J. Elisha Mitchell Sci. Soc. 78: 47-51.

- 1963a. The distribution and general biology of Hyloniscus riparius (Koch) in North America. Crustaceana 8: 131-140.

. 1963b. Philoscia robusta, a new species of terrestrial isopod crustacean from southeastern US. J. Elisha Mitchell Sci. Soc. 79: 26-29.

.1963c. Trichorhina donaldsoni, new species, a terrestrial isopod crustacean from Florida. Amer. Midland Naturalist 69: 435-440.

$1963 \mathrm{~d}$. Venezillo evergladensis, a new species of terrestrial isopod crustacean from Florida. Trans. Am. Micro. Soc. 82 (2): 209-213.

.1964a. Two additional data on terrestrial isopod crustacea: Ligidium blueridgensis spec. nov. from Georgia and North Carolina, cave location for Miktoniscus linearis (Patience, 1908). J. Elisha Mitchell Sci. Soc. 80: 90-94.

- 1964b. Mexiconiscus thamayensis, new genus and new species of terrestrial cave isopod (San Luis Potosí). Trans. Amer. Microsc. Soc. 83: 376-380.

. 1965a. The reduction of Philoscia vittata Say, 1818 to a synonym of Philoscia muscorum (Scopoli, 1793). Crustaceana 8: 107-108.

1965b. Terrestrial Isopods from caves and mines in Texas and northern Mexico with a description of Venezillo tanneri (Mulaik \& Mulaik) Allotype. Texas J. Sci. 17: 101-109.

ecological notes on the new species. Trans. Amer. Microsc. Soc. 85: 457-463. 
1968. The reduction of Pentoniscus Richardson, 1913 to a synonym of Philoscia Latreille, 1804 with notes on disposition of the species (Isopoda: Oniscoidea). Crustaceana 15: 15-18.

. 1969. Anomalous specimens of Philoscia pruinosa (Richardson, 1913) from Costa

Rica.. Rev. Biol. Trop. 16: 129-143.

$.1970 \mathrm{a}$. Redescription of the terrestrial isopod Globarmadillo armatus Richardson, 1910 (Onisciodea: Sphaeroniscidae). Crustaceana 18: 90-92.

-1970b. A review of the genus Tylos Latreille from the New World (Isopoda: Oniscoidea). Crustaceana 19: 297-305.

1970c. Description of new subspecies of Ligidium elrodii with notes on other isopod crustaceans from caves in North America. Amer. Midland Naturalist 84: 36-45.

- 1970d. Disposition of terrestrial isopod crustaceans of the genera Sphaerarmadillo, Sphaeroniscus and Scleropactes (Oniscoidea: Sphaeroniscidae). Proc.

Biol. Soc. Wash. 83: 123-132.

$.1790 \mathrm{e}$. Clyindroniscus vallesensis spec. nov., description with review of the genus

(Isopoda: Trichoniscidae). Trans. Microsc. Soc. 89: 407-412.

- 1972a. The Armadillidae of Florida (Isopoda: Oniscoidea). Quart. J. Florida Acad. Sci. 35: 1-4.

-1972b. Ecology and systematics of terrestrial isopod crustaceans (Oniscoidea) from Bermuda. Crustaceana 3: 79-99.

-1972c. A review of the species of the family Scyphacidae in the New World (Crustacea: Isopoda: Oniscoidea). Proc. Biol. Soc. Wash. 84: 477-488.

1974a. The status of the terrestrial isopod crustaceans Philoscia muscorum, $P h$.

vittata, Ph. robustus, Ph. miamensis in the New World (Oniscidea: Philosciidae).

Crustaceana 27: 147-153.

- 1974b. Terrestrial isopod crustaceans mainly from the West Indies and adjacent regions, 1. Tylos and Ligia. Natuurwet. Studiekr. Sur. Ned. Ant. 77: 162-173.

. 1975. Terrestrial isopod crustaceans from coastal sites in Georgia. Bull. Georgia

Acad. Sci. 34: 185-194.

-1976. Miktoniscus halophilus Blake, M. medcofi (van Name), M. morganensis, new comb. reconsidered with notes on New World species of the genus (Crustacea: Isopoda: Trichoniscidae). Amer. Midland Naturalist 95: 28-41.

. 1977a. Terrestrial isopod crustaceans (Oniscoidea) from St. Catherines Island, Georgia. Georgia J. Sci. 35: 151-158.

- 1977b. Two blind species, one new, of terrestrial isopod crustaceans (Oniscoidea)

from Yucatan and Guatemala. Assoc. Mex. Cave Stud. Bull. 6: 9-13.

. 1981. Isopods from caves in North America and Northern South America. Proc.

8th Internat. Congr. Speleol. 1: 551-552.

- 1982. Amerigoniscus malheurensis, new species, from a cave in western Oregon.

Proc. Biol. Soc. Wash. 95: 89-92.

-1983a. Disposition of three species of Oniscoidea from western Atlantic seashores

(Crustacea: Oniscoidea: Philosciidae and Halophilosciidae). Proc. Biol. Soc. Wash. 96: 440-451.

- 1983b. Two species of Tylos from Chile with notes on species of Tylos with 3 flagellar articles (Isopoda: Oniscoidea: Tylidae). Proc. Biol. Soc. Wash. 96: 675-683

-1984a. Four species of Alloniscus Dana, 1854 from the west coast of North America and Hawaii. Crustaceana 47: 149-167.

- 1984b. Brackenridgia sphinxensis n.sp. from a cave with notes on other species from Arizona and California (Isopoda: Oniscidea). Southwest. Naturalist 29: 309-320 -1984c. Three new and five other species of Oniscoidea from Belize, Central America (Crustacea: Isopoda). J. Nat. Hist. 18: 3-14. 
1994. Typhlotricholigoides and Mexiconiscus from Mexico and Cylindroniscus from North America. J. Crust. Biol. 14: 763-770.

- 1995. Terrestrial isopod crustaceans (Oniscoidea) from Paraguay with definition of a new family. Rev. suisse Zool. 102: 387-424.

Schultz, G.A., R.L. Garthwaite \& C.S. Sassaman 1982. A new family placement for Mauritaniscus littoralis (Miller; 1936) nov. comb. from the West coast of North America with ecological notes (Crustacea: Isopoda: Oniscoidea: Bathytropidae). Wasman J. Biol. 40: 77-89.

Schultz, G.A. \& C. Johnson 1984. Terrestrial Isopod Crustaceans from Florida (Tylidae, Ligiidae, Halophilosciidae. Philosciidae. Rhyscotidae). J. Crust. Biol. 4: 154-171.

SNIDER, R J. 1991. The Michigan isopod fauna. Michigan Acad. 24: 195-200.

SouZA-KURY, L.A. 1993. Notes on Trichorhina, 1. Two new species from northeastern Brazil (Isopoda: Oniscidea: Platyarthridae). Rev. suisse Zool. 100: 197-210.

- 1997a Two new species of Trichorhina from Brazilian Amazonia (Isopoda, Oniscidea, Paltyarthridae). Crustaceana 70 (2): 180-190.

- 1997b. Redescrição e novo registro de Rhyscotus albidemaculatus Budde-Lund, 1908 para o Brasil (Isopoda, Oniscidea, Rhyscotidae). Pap. Avul. Zool. Mus. USP 40 (5): 105-114.

SOuZA, L.A. \& A. Lemos DE CASTRO. 1991. The genus Circoniscus in Brazil, with description of three new species. Trop. Zool. 4: 45-64.

Strouhal, H. 1961. Die Oniscoideen-Fauna der Juan Fernandez-Inseln. Ann. Naturhist. Mus. Wien 48: 185-244.

Taiti, S.; A. Allspach \& F. Ferrara. 1995. A new family placement for the genus Colomboscia with a description of a new species. Stud. Neotrop. Fauna Environ. 30: 91-100.

TAITI, S. \& F. FERrara. 1991a. Two new species of terrestrial Isopoda from Ascension Island. J. Nat. Hist. 25: 910-916.

- 1991b. Terrestrial isopods from the Hawaiian Islands. Bishop Mus. Occ. Pap. 31: 202-227.

TAITI, S.; F. FERRARA \& D.H. Kwon. 1992. Terrestrial Isopods from the Tongian Islands (Sulawesi, Indoniesa). Invertebrate Taxon. 6: 787-842.

- 1986. Chileoniscus marmoratus spec. nov. from Chile. Ann. Hist.-Nat. Mus. Nat. Hung. 78: 63-69.

. 1990. Evolution and biogeography of the family Eubelidae. Biol. Terrestr. Isopods 3: $23-30$.

VANDEL, A. 1945. La répartition géographique des Oniscoidea. Bull. Biol. France Bel. 79: 221-272.

1950. Isopodes terrestres recueillis par C. Bolivar et R. Jeannel (1928) et le Dr. Henrot (1946). Arch. Zool. Exp. Gen. 87: 183-210. $526-529$.

. 1952a. Phalloniscus boliviamus, n.sp.. Bull. Mus. Natl. Hist. Nat., 2. Ser., 24: 1952b. Étude des isopodes terrestres recoltés au Venezula par le Dr. G. Marcuzzi. Mem. Mus. Civ. Stor. Nat. 3: 59-201.

1952c. La répartition du complexe trichoiniscoide et les théories géologiques.

Comp. rend. sean. Acad. Sci. Paris 235: 997-999.

6: $1-116$.

1953. Les Trichoniscoides de l'hémisphère australe. Mem. Mus. Natl. Hist. Nat. . 1956. Remarques complémentaires et rectifications relatives à Trichorhina boliviana Vandel, 1952. Bull. Mus. Natl. Hist. Nat., 2. Ser., 28: 300-302.

1963. Isopodes terrestres receuillis en Amérique du Sud par C.D. Deboutteville.

Biol. Amer. Austr. 2: 63-100. 
1965a. Sur l'existence d'oniscoides très primitifs menant une vie aquatique et sur

le polyphylétisme des isopodes terrestres. Ann. Speleol. 20: 489-518.

. 1965b. Les Trichoniscidae cavernicoles de l'Amérique du Nord. Ann. Speleol. 20: 348-389.

1968. Isopodes terrestres. Miss. zool. belge Galapagos Ecuador 84: 35-168.

1970. Une troisième oniscoide cavernicole menant une vie aquatique:

Mexiconiscus laevis (Rioja). Ann. Speleol. 25: 161-171.

.1972a. Les isopodes terrestres de la Colombie. Stud. Neotrop. Fauna Environ.

7: $147-172$.

1972b. De l'utilisation des données biogéographiques dans la reconstitution des

anciens visages du globe terrestre. Comp. rend.sean. Acad. Sci.Paris Ser. D 271: 38-41.

. 1973. Les isopodes terrestres et cavernicoles de l'île de Cuba. Res. exp. biospel. cub.-rom. Cuba 1: 153-188.

1977. La faune terrestre le l'Île Ste. Hélène: Isopodes terrestres. Ann. Mus. r.

Afr. Centr. Ser. 8vo 220: 385-426.

1978. Les espèces appartenant au genre Amerigoniscus Vandel, 1950. Bull. Soc.

Hist. Nat. Toulouse 113: 303-31.

. 1981. Les isopodes terrestres et cavernicoles de l'Île de Cuba. Res. exp. biospel. cub.-rom. Cuba 3: 35-76.

VAN KLINKEN, R.D. \& A.J.A. GREEN. 1992. First record of Oniscidea from Macquarie Island.

Polar Rec. 20: 240-242.

VAN NAME, W.G. 1926. Forest isopods from Barro Colorado Island, Panama Canal Zone.

Amer. Mus. Nov. 206: 1-25.

-1936. American terrestrial and fresh water Isopoda. Bull. Amer. Mus. Nat. Hist. 71: $1-520$.

1940. A supplement to the American terrestrial and fresh water Isopoda. Bull. Amer. Mus. Nat. Hist. 77: 109-142.

1942. A second supplement to the American terrestrial and fresh water Isopoda.

Bull. Amer. Mus. Nat. Hist. 80: 299-329.

VERHOEFF, K.W. 1926. Isopoda terrestria von Neu Caledonien und den Loyality Inseln, In:

Nova Caledonia, A 4: 241-364.

1928. Über einige Isopoden der zoologischen Staatssammlung in München. Zool. Anz. 76: 25-36 and 113-123.

97-119.

1933. Neue Isopoden aus Mexiko und dem Mediterrangebiet. Zool. Anz. 103:

. 1939. Von Dr. G.H. Schwabe gesammelte Isopoda terrestria, Diplopoda und Chilopoda. Zeitschr. f. wiss. Zool. 8: 301-324.

- 1941a. Über eine neue südamerikanische Gattung der Isopoda Terrestria. Zool.

Anz. 134: 169-173.

1941b. Landisopoden. in: Titschack (Ed.) Beiträge zur Fauna Perus 2: 74-80.

. 1941 c. Zur Kenntnis südamerikanischer Oniscoideen. Zool. Anz. 133: 114-126.

4: $1-19$.

Vilela, E.F.; H. Kudo \& M. Loureiro. 1971. Oniscoides de Dourdados, Estado de Mato Grosso. Seiva 31: 183-189.

ZARDO, C.M.L. 1989. Uma nova espécie de Phalloniscus Budde-Lund, 1908 do sul do Brasil.

Rev. Brasil. Biol. 6: 611-615.

Zardo, C.M.L. \& J. Loyola E Silva. 1988. Primeira ocorrência de Oniscus asellus Linné,

1758 e Porcellionides sexfasciatus (Koch, 1847) no Brasil (Isopoda: Oniscoidea).

Ciência e Cultura 40: 791-779.

Recebido em 10.X.1997; aceito em 20.III.1999. 


\section{INDEX}

acapulcensis, Cubaris

acostai, Brackenridgia

acostai, Protrichoniscus (syn.)

acuta, Trichorhina

advena, Porcellionides.

Agabiformius....

Agnara

aguayoi, Cubaris (syn.)

aguayoi, Venezillo

aiasensis, Platyarthrus.

alabamensis, Miktoniscus (syn.).

albamaculata, Prosekia

albidemaculatus, Rhyscotus........................ 32

albomarginata, Benthana ......................... 13

Alboscia.................................................... 12

alceui, Atlantoscia (syn.) .......................... 13

Alloniscus ................................................ 11

alticola, Colombophiloscia ......................... 16

alticola, Helenoscia (syn.)......................... 18

alticola, Littorophiloscia ............................ 18

alticolus, Clavigeroniscus............................. 8

amazonica, Ischioscia................................ 16

amazonica, Proischioscia (syn.)................ 16

amazonica, Trichorhina.............................. 28

amazonicus, Circoniscus ........................... 37

Amazoniscus.................................................. 37

ambigua, Trichorhina ................................ 28

americanum, Ethelum ................................ 42

Amerigoniscus .............................................. 4

Andenoniscus ............................................. 12

andina, Ischioscia .................................... 17

andina, Proischioscia (syn.) ..................... 17

andinus, Scleropactes .................................. 40

Andronsicus .................................................... 5

angulatus, Pectenoniscus........................... 9

angulatus, Thomasoniscus .........................223

angusta, Pseudophiloscia .......................... 23

angustata, Benthana .................................. 13

anomala, Oniscophiloscia ........................ 19

anomala, Philoscia (syn.) ........................... 19

anomalus, Phalloniscus (syn.).................. 19

antarctica, Sphaerobathytropa .................. 41

apachea, Cubaris (syn.)............................. 46

apacheus, Venezillo ....................................... 46

apenensis, Circoniscus ............................... 37

apeuensis, Parsphaeroniscus (syn.) .......... 37

aquaticus, Typhlotricholigoides .................. 8

araucanicus, Styloniscus.
Araucoscia ................................................ 13

Archaeosia .................................................. 13

arenicola, Scyphacella ........................... 12

argentina, Philoscia (syn.) ........................ 31

argentina, Trichorhina ........................... 28

argentinus, Alloniscus (syn.) ..................... 31

argentinus, Neotroponiscus ....................... 26

argentinus, Pardioniscus (syn.).................. 31

argentinus, Plataoniscus (syn.) ................. 31

argentinus, Porcellio (syn.)....................... 26

Arhina ........................................................ 13

arizonicus, Cubaris (syn.) ........................ 46

arizonicus, Venezillo.................................. 46

arlei, Amazoniscus .................................... 37

Armadillidae .............................................. 43

Armadillidiidae ........................................... 43

Armadillidium ......................................... 43

Armadillo .......................................................... 46

Armadilloniscus ........................................... 11

armatus, Globarmadillo ............................ 44

armatus, Oniscus ........................................ 24

armatus, Synarmadillo (syn.) ................... 44

articulatus, Armadillo (syn.) ..................... 46

articulatus, Venezillo .................................. 46

asellus, Oniscus ...................................... 34

atlantica, Laureola ...................................... 45

Atlantoscia .................................................. 13

atoyacensis, Trichorhina ........................... 28

avrilensis, Phalloniscus ............................ 25

avrilensis, Philoscia (syn.) ....................... 25

Baconaoscia.............................................. 13

balamensis, Antroniscus (syn.).................... 6

baldoni, Phalloniscus ................................ 25

baldoni, Philoscia (syn.)......................... 25

Balloniscidae.............................................. 31

Ballonsicus ................................................. 31

barbouri, Phalloniscus (syn.)................... 26

barbouri, Trichorhina (syn.) ................... 26

barrai, Miktoniscus ..................................... 7

Bathytropidae.............................................. 26

baudiniana, Ligia ....................................... 2

beebei, Cubaris (syn.)................................ 46

beebei, Venezillo ........................................ 46

belizensis, Troglophiloscia ......................... 23

bellavistanus, Venezillo .............................. 46

benitensis, Cubaris ................................... 44

Benthana ................................................ 13

Benthanoides ............................................... 15 
Benthanoscia........................................... 15

bequaerti, Trichorhina ............................. 28

bermudensis, Littorophiloscia ................... 18

bermudezi, Porcellionides ........................... 35

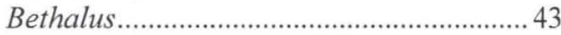

bezzi, Circoniscus ...................................... 38

bicolor, Microspaeroniscus .......................... 39

bicolor, Trichorhina.................................. 28

bilineata, Benthana ................................... 13

birabeni, Pudeoniscus................................ 43

Bisilvestriidae .............................................. 37

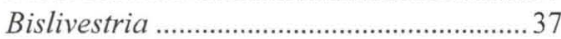

bituberculata, Colomboscia........................ 38

blueridgensis, Ligidium .............................. 3

bocainensis, Benthana .............................. 13

bodkini, Calycuoniscus .............................. 24

bolivari, Ischioscia...................................... 17

bolivari, Cubaris ......................................... 44

boliviana, Trichorhina ............................... 29

bolivianus, Armadillo (syn.) ....................... 46

bolivianus, Phalloniscus (syn.).................. 29

bolivianus, Venezillo .................................... 46

bonarensis, Philoscia................................ 20

boneti, Armadillo (syn.).............................. 46

boneti, Trichorhina ...................................29

boneti, Venezillo .............................................. 46

bonitanus, Sphaeroniscus ........................... 41

boonae, Cubaris (syn.) ............................. 47

booneae, Venezillo ...................................... 47

borellii, Alloniscus (syn.)........................... 31

borellii, Plataoniscus .................................. 31

botosaneanui, Scleropactes ....................... 40

Brackenridgia ................................................ 5

brasiliensis, Halophiloscia (syn.)........ 14, 24

brasiliensis, Trichorhina.

Brasilocellio (syn.).

Brasiloniscus .

brevicornis, Ballonsicus............................ 31

brevicornis, Cubaris (syn.)......................... 47

brevispinis, Venezillo ................................... 47

briani, Cubanophiloscia ........................... 16

briani, Philoscia (syn.) ............................. 16

bridgesi, Brackenridgia .............................. 5

bridgesi, Protrichoniscus (syn.) ................. 5

brunea, Cubaris (syn.)............................... 44

brunneus, Porcellionides ............................ 35

bucculenta, Deto .......................................... 12

Burmoniscus............................................ 15

buschki, Pseudarmadillo............................ 52

cacahuampilensis, Armadillo (syn.) .......... 47 cacahuampilensis, Cubaris (syn.) ............ 47

cacahuampilensis, Venezillo..................... 47

caeca, Trichorhina.................................... 29

caelata, Eluma .............................................. 43

cajennensis, Ligia ........................................... 2

californicus, Venezillo ................................ 49

callani, Ligia (syn.) ..................................... 3

Calycuoniscus .............................................. 24

capensis, Niamba ........................................ 27

caraibicus, Armadilloniscus ....................... 11

Caraiboscia ................................................ 15

carniculatus, Pseudarmadillo .................... 52

caroli, Neotroponiscus ............................... 27

Caucasonethes (syn.)..................................... 5

cavernarum, Brackenridgia......................... 5

cavernarum, Protrichoniscus (syn.) ............ 5

cavernicola, Colombophiloscia .................. 16

cavernicola, Spherarmadillo ...................... 41

cavicolus, Antroniscus (syn.)...................... 6

cavicolus, Cylindroniscus............................ 6

cavifrons, Scleropactes ............................. 40

cayennensis, Porcellio (syn.)...................... 34

cedrosensis, Scleropactes (syn.)................ 11

centralis, Amerigoniscus ............................... 4

Chaetophiloscia ........................................ 15

chamberlini, Cubaris (syn.)...................... 47

chamberlini, Venezillo ................................ 47

chiapensis, Venezillo .................................. 47

chilenica, Araucoscia ................................. 13

chilensis, Tylos.............................................. 4

Chileoniscus.................................................. 37

ciferrii, Rhyscotoides .................................. 32

ciferrii, Rhyscotus (syn.)............................ 32

cinchonae, Cubaris ...................................... 44

cineraea, Cubaris ...................................... 44

cinerascens, Ligia ........................................ 2

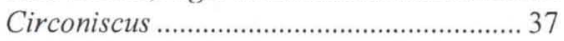

clausa, Cubaris (syn.)............................... 45

clausus, Armadillo (syn.)............................ 45

clausus, Synarmadillo.................................. 45

Clavigeroniscus ............................................ 8

coecus, Elumoides ...................................... 42

colimensis, Philoscia ................................. 20

colimensis, Rhyscotus ................................ 32

colomboi,Cubaris (syn.) ............................ 47

colomboi, Venezillo .................................... 47

Colomboniscus........................................... 38

Colombophiloscia ........................................ 15

Colomboscia .................................................... 38

columbiensis, Parsphaeroniscus (syn.).... 40 
columbiensis, Scleropactes ......................... 40

columbiensis, Sphaeroniscus (syn.).......... 40

compar, Alloniscus (syn.) ................... 18, 24

compar, Calycuoniscus............................. 24

concinnus, Scleropactes.............................. 40

congener, Armadillo (syn.) ......................... 47

congener, Venezillo.................................... 47

congenera, Cubaris (syn.) ......................... 47

contogensis, Philoscia ................................ 20

contogensis, Stenoniscus ............................ 11

convexa, Benthana .................................... 13

convexus, Cylisticus .................................. 36

cordillerae, Colomboscia ............................ 39

Cordioniscus ................................................ 9

cornutus, Alloniscus (syn.) ......................... 11

coronacapitalis, Armadilloniscus .............. 11

Cosmeodillo .................................................. 43

costaricensis, Mirtana ............................... 19

costatus, Microspaeroniscus...................... 39

couchii, Halophiloscia ................................ 24

Coxopodias (syn.) ....................................... 46

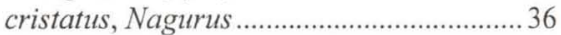

cristatus, Pseudarmadillo ......................... 52

ctenoscoides, Philoscia ..............................20

cubanocolus, Nagurus ................................ 36

Cubanophiloscia ….................................... 16

Cubanoscia ..................................................26

Cubaris ......................................................... 44

cubensis, Rhyscotoides................................ 32

cubensis, Rhyscotus (syn.) ......................... 32

culebrae, Cubaris (syn.).............................. 47

culebrae, Littorophiloscia......................... 18

culebrae, Venezillo........................................ 47

culebroides, Nesophiloscia ....................... 19

culebroides, Philoscia (syn.) ..................... 19

curvatus, Amerigoniscus ................................ 4

Cylindroniscus ............................................. 6

Cylisticidae ................................................ 36

Cylisticus ........................................................ 36

daguerrei, Neotroponiscus ........................ 27

daguerrei, Porcellio (syn.) ......................... 27

danicus, Haplophthalmus ............................ 6

davisi, Porcellionides (syn.) ...................... 33

decoui, Cosmeodillo..................................... 43

decoui, Pacroscia......................................... 19

delamarei, Dubioniscus ............................... 24

demerarae, Philoscia ................................. 21

demivirgo, Trichoniscus ............................. 8

dentiger, Andronsicus ................................... 5

dentiger, Trichoniscus (syn.) ....................... 5 depressus, Bethalus ................................... 43

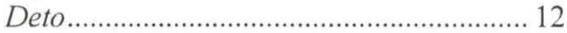

Detonella......................................................... 12

dilatatus, Porcellio ...................................... 33

dilatatus, Stymphalus..................................... 3

diminuta, Philoscia ..................................... 21

dimorpha, Benthana ................................ 14

dimorpha, Pagana ...................................... 37

Diploexochus .............................................. 44

dissimilis, Novamundoniscus................... 25

dissimilis, Phalloniscus (syn.) .................. 25

dollfusi, Pseudarmadillo............................ 52

dominicensis, Pentoniscus ......................... 20

dominicensis, Philoscia (syn.).................. 20

dominicensis, Rostrophiloscia ................... 23

donaldsoni, Trichorhina ............................. 29

Dubioniscidae ................................................ 24

Dubioniscus .................................................. 24

duffreyi, Niamba ...................................... 28

dugesi, Armadillo (syn.) ............................ 47

dugesi, Cubaris (syn.)............................... 47

dugesi, Venezillo ......................................... 47

dumorum, Armadillo (syn.) ...................... 47

dumorum, Cubaris (syn.).......................... 47

dumorum, Venezillo ................................... 47

echinatus, Diploexochus ........................... 44

Ecuadoroniscus ........................................... 16

ellipticus, Armadilloniscus ........................ 11

elongata, Alboscia ..................................... 12

elongata, Ischioscia ................................ 17

elongata, Pacroscia .................................. 19

elrodii, Ligidium ....................................... 3

Eluma .......................................................... 43

Elumoides .................................................... 42

epigea, Suleoscia ........................................ 23

Erophiloscia.............................................. 16

esterelanus, Cylisticus ................................ 36

estherae, Scleropactes .............................. 40

Ethelum ........................................................ 42

Eubelidae .................................................... 42

evergladensis, Venezillo (syn.) ................... 49

exilis, Pentoniscus ....................................... 20

exilis, Philoscia (syn.) ........................... 20

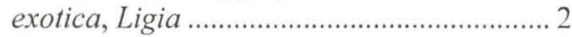

fernandezi, Notoniscus ............................... 9

filicornis, Ligia ............................................. 2

flagellata, Tropiscia................................. 23

flavobrunnea, Cubaris ............................. 44

flavomaculatus, Sphaeroniscus ................. 41

flavovittata, Porcellionides (syn.) ............. 35 
floria, Porcellionides ................................. 35

floridana, Atlantoscia .............................. 13

floridana, Ocelloscia (syn.) ....................... 13

floridana, Philoscia (syn.) ........................ 13

floridanum, Ligidium ...................................... 3

Floridoscia .................................................. 16

formosae, Philoscia ...................................21

frontalis, Chaetophiloscia.......................... 15

frontalis, Sphaeroniscus ............................ 41

fuegensis, Porcellionides ............................ 35

fusca, Floridoscia ........................................ 16

gaigei, Circoniscus .................................... 38

gaigei, Scleropactes .................................... 40

gaigei, Sphaeroniscus (syn.)..................... 40

galapagensis, Chaetophiloscia (syn.)........22

galapagensis, Prosekia .............................. 22

galapagoensis, Cubaris (syn.) .................... 48

galapagoensis, Venezillo .......................... 48

gatunensis, Chaetophiloscia ...................... 15

gatunensis, Philoscia (syn.)...................... 15

geayi, Philoscia .......................................... 21

geiseri, Philoscia....................................... 21

gemmulatus, Porcellio (syn.).................... 34

georgiensis, Amerigoniscus ......................... 4

gerstaeckeri, Sphaeroniscus ...................... 41

gertschi, Porcellio (syn.) ........................... 33

giambiagiae, Laninoniscus ........................ 26

gianelli, Trichorhina ................................29

gibbus, Pseudodiploexochus..................... 45

gibbus, Reductoniscus (syn.) .................... 45

gigas, Cubaris (syn.)................................. 48

gigas, Venezillo ......................................... 48

gillianus, Pseudarmadillo .......................... 52

gipsicolus, Amerigoniscus .......................... 5

gipsicolus, Caucasonethes (syn.)................. 5

glaber, Ballonsicus ..................................... 31

Globarmadillo............................................ 44

goeldii, Calycuoniscus (syn.)..................... 24

goeldii, Dubioniscus ................................. 24

goeldii, Hileioniscus (syn.) ........................ 24

gracile, Ligidium ........................................... 3

gracilidens, Circoniscus ........................... 38

gracilior, Philoscia ..................................... 21

granaria, Cubaris ....................................... 44

granarus, Porcellio ..................................... 33

granulatus, Leptotrichus (syn.) .................. 33

granulatus, Scleropactes............................ 40

granulatus, Sphaeroniscus........................ 41

granulatus, Synuropus (syn.).................... 40

grayi, Miktoniscus (syn.) ........................... 7 grenadensis, Armadillo (syn.) .................. 48

grenadensis, Cubaris (syn.)..................... 48

grenadensis, Venezillo ............................... 48

griseus, Alloniscus (syn.).......................... 31

griseus, Plataoniscus ................................. 31

guanophila, Trichorhina .......................... 29

guerrerense, Philoscia.............................. 21

guianensis, Sphaeroniscus....................... 41

habanensis, Porcellionides ....................... 35

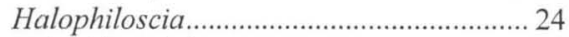

Halophilosciidae .......................................... 24

halophilus, Miktoniscus (syn.).................... 7

hamatus, Circoniscus ................................ 38

hamigera, Chaetophiloscia (syn.) ............. 22

hamigera, Prosekia .................................. 22

hanagarthi, Ischioscia .............................. 17

Haplarmadillo (syn.) .................................. 46

Haplophthalmus ......................................... 6

hawaiensis, Ligia ............................................ 2

Helenoscia (syn.) ...................................... 18

hendersoni, Cubaris (syn.) ........................ 48

hendersoni, Venezillo................................. 48

henroti, Amerigoniscus .................................... 5

heroldi,Caucosonethes (syn.) ...................... 5

heroldi, Brackenridgia ................................ 6

heroldi, Protrichoniscus (syn.)................... 6

heteroclita, Jimenezia ............................... 18

heterophthalma, Trichorhina .................... 29

hildaguensis, Porcellionides (syn.) ........... 33

Hileioniscus (syn.) .................................... 24

hoctuni, Trichoniscus ................................. 8

Hoctunus ....................................................... 16

hoffmannseggi, Platyarthrus ..................... 28

holmesi, Armadilloniscus .......................... 11

holpites, Delatorella (syn.)....................... 52

holpites, Pseudarmadillo ........................... 52

huatuscensis, Spherarmadillo ................... 42

humus, Miktoniscus (syn.) ............................ 7

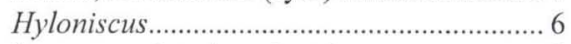

hypnorum, Ligidium (syn.) ....................... 3

iheringi, Styloniscus..................................... 9

incerta, Philoscia ....................................... 21

incisus, Circoniscus ..................................... 38

incisus, Scleropactes................................. 40

inflexa, Pseudophiloscia ............................. 23

inquilina, Philoscia................................... 21

insularis, Dubioniscus ............................. 24

insularis, Prosekia ..................................... 22

insularis, Pseudodiploexochus ................. 45

insularis, Reductoniscus (syn.)................. 45 
insularis, Tylos

insularuminfraventum, Ballonsicus ............31

intermedius, Circoniscus ........................... 38

iporangensis, Benthana ............................ 14

irmleri, Ischioscia ..................................... 17

Ischioscia .................................................... 16

isthmica, Trichorhina............................... 29

jacksoni, Rhyscotus ................................. 32

jamaicensis, Cubaris (syn.) ....................... 48

jamaicensis, Venezillo .............................. 48

jelkinsi, Porcellionides (syn.) ..................... 35

Jimenezia...................................................... 18

kartaboana, Philoscia .............................. 21

kofoidi, Ligidium .......................................... 3

kuscheli, Oniscophiloscia ......................... 19

Kuscheloniscus.............................................. 9

laevis, Cordioniscus (syn.) ......................... 7

laevis, Mexiconiscus ..................................... 7

laevis, Porcellio ............................................. 34

laevis, Troglophiloscia.............................. 23

laevis, Xilitloniscus (syn.)............................ 7

lamellatus, Porcellio .................................. 34

langi, Phalloniscus.................................... 26

langi, Philoscia (syn.)............................. 26

Laninoniscus ................................................26

lapetum, Ligidium ....................................... 3

lativentris, Oreades ..................................... 19

latreillei, Tylos ............................................... 4

latum, Ligidium .............................................. 3

Laureola .......................................................... 45

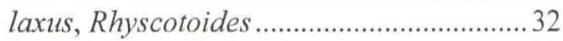

laxus, Rhyscotus (syn.) ............................. 32

lejeunei, Prosekia..................................... 22

leleupi, Cordioniscus .................................... 9

leleupi, Pseudodiploexochus....................... 45

leleupi, Reductoniscus (syn.) ..................... 45

lenkoi, Neotroponiscus .............................27

lentus, Agabiformius .................................... 33

Leptotrichus ................................................... 33

Ligia .............................................................. 2

Ligidium ................................................. 3

Ligiidae ....................................................... 2

liliputanus, Porcellio ................................... 34

lindahli, Armadilloniscus ........................... 11

linearis, Miktoniscus (syn.).......................... 7

littoralis, Neotroponiscus ..........................27

littorinus, Mauritaniscus (syn.) .................27

littorinus, Porcellio (syn.).......................... 27

Littorophiloscia............................................ 18

llamasi, Venezillo ......................................... 48 lobatus, Neotroponiscus ........................... 27

lobifera, Ischioscia (syn.) ......................... 17

lomanderi, Detonella (syn.)...................... 12

longiantennata, Niamba .......................... 28

longicauda, Ischioscia .............................. 17

longicaudata, Benthanoscia ...................... 15

longicaudatum, Ligidium (syn.) .................. 3

longicornis, Benthana ............................. 14

longipenis, Benthana ............................... 14

longispinis, Cubaris (syn.) ...................... 48

longispinis, Venezillo ................................. 48

longistyla, Erophiloscia .............................. 16

loyolai, Phalloniscus ................................ 26

macrophthalma, Trichorhina .................... 29

macrophthalmus, Novamundoniscus........ 25

macrophthalmus, Phalloniscus (syn.)...... 25

macrops, Trichorhina ............................... 29

macrosoma, Armadillo (syn.)................... 48

macrosoma, Venezillo ............................... 48

maculatus, Ballonsicus .............................. 31

maculatus, Brasiloniscus .......................... 42

madagascariensis, Agnara ........................ 36

magellanicus, Styloniscus ......................... 10

magellanicus, Trichoniscus (syn.)............. 10

malheurensis, Amerigoniscus ..................... 5

marcuzzi, Novamundoniscus ................... 25

marcuzzi, Phalloniscus (syn.)................... 25

marcuzzii, Tylos ............................................. 4

margaritae, Cubaris ................................. 44

marginalis, Porcellio ................................ 34

mariani, Trichorhina ................................ 29

marina, Deto ................................................ 12

marmoratus, Chileoniscus .......................... 37

marmoratus, Dubioniscus ......................... 25

marrassinii, Bislivestria............................. 37

martinae, Ischioscia................................... 17

Mauritaniscus (syn.)................................. 27

maya, Cylindroniscus ................................ 6

medcofi, Miktoniscus .................................... 7

meeusei, Burmoniscus ............................. 15

mellissi, Pseudodiploexochus ................... 45

mellissi, Reductoniscus (syn.).................... 45

meridionalis, Phalloniscus ........................ 26

Metastenoniscus......................................... 10

mexicana, Cubaris (syn.)......................... 48

mexicanus, Microdillo (syn.)...................... 48

mexicanus, Venezillo (syn.) ...................... 48

Mexiconiscus ................................................ 7

Mexicostylus (syn.) .................................... 30

miamensis, Philoscia (syn.)...................... 18 
Microdillo 48

Microphiloscia

microphthalma, Caraiboscia ..................... 15

microphthalma, Cubaris (syn.)................. 48

microphthalmus, Armadillo (syn.)............. 48

microphthalmus, Venezillo ......................... 48

Microspaeroniscus .................................... 39

Miktoniscus ....

mineri, Cubaris (syn.)............................... 49

mineri, Ischioscia ..................................... 17

mineri, Philoscia (syn.)............................ 17

mineri, Venezillo .......................................... 49

minuta, cubaris ......................................... 44

minutissimus, Porcellionides ..................... 35

mirabilis, Alloniscus ................................. 11

mirabilis, Xiphoniscus ............................... 23

mirandai, Cubaris ..................................... 44

mirifica, Oniscophiloscia.......................... 19

Mirtana ..................................................... 19

modestum, Ethelum .................................... 42

modestus, Agabiforminus............................... 33

modestus, Lyprobius (syn.)........................ 33

moneaguensis, Cubaris (syn.) ................... 49

moneaguensis, Philoscia............................21

moneaguensis, Venezillo ............................. 49

monocellatus, Oligoniscus (syn.)............... 10

monocellatus, Styloniscus .......................... 10

monocullatus, Haplarmadillo (syn.).......... 46

monocullatus, Synarmadillo ....................... 46

moreirai, Benthana ..................................... 14

morganensis, Miktoniscus............................ 7

mucronatum, Ligidium ................................ 3

muelleri, Ischioscia .................................. 17

mulaiki, Porcellionides (syn.).................... 36

multipunctata, Cubaris (syn.)................... 49

multipunctatus, Armadillo (syn.) ............... 49

multipunctatus, Venezillo........................... 49

murina, Cubaris ......................................... 44

murrayi, Styloniscus................................... 10

muscorum, Ligia (syn.) ................................ 3

muscorum, Philoscia (syn.) ................ 17, 21

naevigesta, Colombophiloscia................... 16

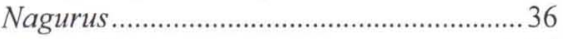

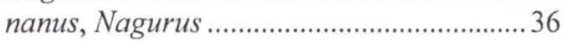

narcissi, Andenoniscus (syn.) ................... 16

narcissi, Erophiloscia ................................ 16

nasatum, Armadillidium ............................. 43

nasutus, Rhyscotus .................................... 32

nearcticus, Oregoniscus .............................. 7

nearcticus, Trichoniscus (syn.)................... 7 negreae, Dubioniscus .............................. 25

negreai, Baconaoscia ............................... 13

negreai, Parapacroscia ............................. 19

Neosanfilippia ........................................... 39

neotropicalis, Metastenoniscus ................ 10

Neotroponiscus ......................................... 26

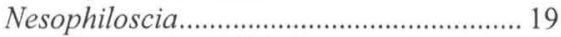

nevadensis, Armadillo (syn.) ..................... 49

nevadensis, Venezillo ................................. 49

Niamba ....................................................... 27

nicholasi, Amerigoniscus ............................. 5

nigrescens, Oniscus (syn.)......................... 14

nigricans, Ballonsicus ............................. 31

nigrorufa, Cubaris (syn.).......................... 49

nigrorufus, Armadillo (syn.)...................... 49

nigrorufus, Venezillo .................................. 49

ninae, Armadilloniscus ............................. 12

nitida, Ischioscia ........................................ 17

nitida, Philougria (syn.) ........................... 17

niveus, Tylos ................................................ 4

nodulosus, Brasilocellio (syn.).................. 26

nomae, Littorophiloscia............................ 18

nordenskjoldi, Styloniscus ........................ 10

Notoniscus ................................................... 9

novaezealandiae, Ligia ................................. 2

Novamundoniscus ...................................... 25

oaxacana, Cubaris (syn.) .......................... 49

oaxacanus, Venezillo .................................. 49

obscurus, Pudeoniscus .............................. 43

occidentalis, Ligia ........................................ 2

oceanica, Ligia ............................................... 2

ohioensis, Miktoniscus (syn.) ..................... 7

oklahomensis, Miktoniscus .......................... 7

olfersi, Benthana.......................................... 14

olfersi, Ligia (syn.) ....................................... 2

olfersi, Philoscia (syn.)............................. 14

omissa, Parischioscia ................................ 20

omissa, Philoscia (syn.)............................ 20

Oniscidae .................................................. 24

Oniscophiloscia ............................................ 19

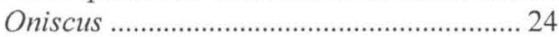

orchidicola, Trichoniscus ........................... 8

Oreades......................................................... 19

Oregoniscus ...................................................... 7

orghidani, Clavigeroniscus ......................... 9

orientalis, Ecuadoroniscus ....................... 16

ornatus, Parsphaeroniscus (syn.)............. 38

orosioi, Armadillo (syn.) ........................... 49

orosioi, Venezillo ........................................ 49

orthonedae, Rhyscotoides........................... 32 
orthonedae, Rhyscotus (syn.).. otakenssis fernandeziamus, Styloniscus ..... 10

Pacroscia

Pagana .. 37

pallasii, Ligia ..3

pallidus, Circoniscus ... 38

pallidus, Microspaeroniscus.

39

pallidus, Styloniscus.

10

panzeri, Leptotrichus (syn.)

33

papillicornis, Detonella papillosa, Trichorhina

Paracubaris (syn.)

paraensis, Trichorhina

Paraguascia

paraguayana, Philoscia (syn.)

paraguayanus, Balloniscus.....

parallelus, Rhyscotoides

parallelus, Rhyscotus (syn.).

Parapacroscia

Pardioniscus (syn.)

Parischioscia.....

Parsphaeroniscus (syn.)....

parvus, Sphaerillo...

parvus, Venezillo

paulensis, Chaetophiloscia (syn.).

paulensis, Philoscia (syn.).

pauper, Benthanoides

paynesi, Cancasonethes (syn.).

pearsei, Chaetophiloscia (syn.).

pearsei, Phalloniscus

pearsei, Philoscia (syn.)

pearsei, Porcellio (syn.).....

pearsei, Prosekia

pearsei, Trichorhina

Pectenoniscus.

Pentoniscus

perconvexus, Alloniscus

Periscyphis.

perlatus, Armadillo (syn.)

perlatus, Cubaris (syn.).

perlatus, Neotroponiscus

perlatus, Venezillo

persimilis, Novamundoniscus .....

persimilis, Phalloniscus (syn.).

peruensis, Benthana

peruvianus, Sphaeroniscus

Phalloniscus.

Philoscia

Philosciidae

phylax, Cubaris (syn.).

12

29

..38

.29

19

31

31

32

32

19

31

20

38 phylax, Venezillo 50

picta, Benthana..... 14

pigmentata, Paraguascia.

pilosus, Scleropactes

pilosus, Sphaeroniscus

pisum, Armadillo (syn.)

pisum, Venezillo.....

pittieri, Trichorhina

Pittieroniscus 39

Plataoniscus _............................................... 31

Platyarthridae .............................................. 27

Platyarthrus .......................................... 28

platycephala, Ligia ..................................... 3

plaumanni, Brasilocellio (syn.) ................. 27

plaumanni, Neotroponiscus....................... 27

pleogoniphora, Cubaris (syn.)................... 50

pleogoniphorus, Venezillo .......................... 50

pleonalis, Stenoniscus.................................. 11

Porcellio ……………………………....... 33

porcellioides, Arhina ……………………... 13

Porcellionidae ......................................... 33

Porcellionides ………………………........ 35

portoricensis, Richardsoniscus .................. 39

portoricensis, Sphaeroniscus (syn.) .......... 39

potosinus, Protrichoniscus (syn.)................ 5

primitiva, Cubanoscia ………………….... 26

Proporcellio ………………………..... 36

Prosekia ................................................... 22

Protosphaeroniscus ................................... 39

provisorius, Trichoniscus ............................ 8

proxima, Cubanoscia ................................. 26

proximus, Amerigoniscus ............................. 5

pruinosa, Philoscia (syn.).......................... 20

pruinosus, Pentoniscus .............................. 20

pruinosus, Porcellionides ........................... 35

Pseudarmadillidae....................................... 52

Pseudarmadillo ........................................ 52

Pseudodiploexochus ……………………... 45

Pseudophiloscia ........................................ 23

pseudopusillus, Trichoniscus....................... 8

pubescens, Porcellio ................................... 34

Pudeoniscidae .......................................... 42

Pudeoniscus ............................................. 43

pumila, Cubaris (syn.) ................................ 50

pumilus, Venezillo ..................................... 50

punctatus, Tylos ………………………...... 4

pusillus, Agabiformius ............................... 33

pusillus, Lyprobius (syn.) ........................... 33

pusillus, Trichoniscus ................................... 8

Puteoscia ................................................. 23 
pygmaeus, Trichoniscus............................ 8 quadrifrons, Porcellio (syn.) ...................... 34 quadriseriatus, Proporcellio......................... 36 quisquiliarum, Trichorhina......................... 30 racovitzai, Miktoniscus .................................. 7

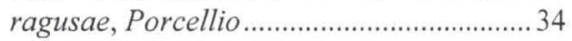
ramsdeni, Cubaris (syn.) .......................... 48 rathkei, Trachelipus .................................... 37 reddelli, Brackenridgia ................................ 6 reddelli, Protrichoniscus (syn.) ....................6 Reductoniscus (syn.) .................................... 45 reflexum, Ethelum ...................................... 42 regressus, Colomboniscus ......................... 38

Rhabdoniscus ............................................. 27

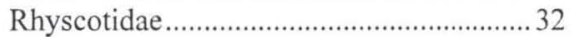

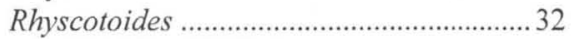

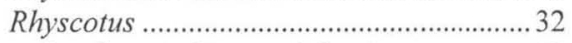

richardsonae, Littorophiloscia .................. 18

richardsonae, Philoscia (syn.)................... 18

richardsonae, Trachelipus ........................ 37

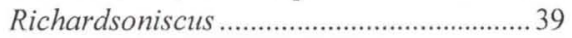

richmondi, Philoscia .................................. 21

riedli, Vandeloscia (syn.)........................... 18

riparius, Hyloniscus .................................... 6

riqueri, Clavigeroniscus ............................. 9

robusta, Philoscia (syn.).......................... 18

robustus, Rhabdoniscus ............................. 27

romanorum, Colombophiloscia ................. 16

romanorum, Cubanoscia ............................26

romanorum, Styloniscus ........................... 10

roraimae, Philoscia ................................... 21

Rostrophiloscia ............................................ 23

rothi, Amerigoniscus ...................................... 5

rothi, Caucasonethes (syn.)......................... 5

rubropunctata, Cubaris (syn.) ................... 50

rubropunctatus, Venezillo.......................... 50

ruthveni, Coxopodias (syn.)....................... 46

ruthveni, Synarmadillo ............................... 46

rutilans, Chaetophiloscia (syn.) ................ 22

rutilans, Prosekia ......................................... 22

salinarum, Alloniscus................................ 11

sanchezi, Cubaris (syn.) .......................... 50

sanchezi, Venezillo ........................................ 50

santosi, Benthana ......................................... 14

sarsi, Trichoniscoides .................................... 8

saussurei, Porcellionides ............................ 35

scaber, Porcellio ............................................. 34

scaberrima, Cubaris (syn.) ........................ 50

scaberrimus, Armadillo (syn.) .................... 50

scaberrimus, Venezillo ................................ 50 scabrisculus, Porcellio .............................. 34

schoeblii aiasensis, Platyarthrus............... 28

schubarti, Benthana................................... 14

schultzei, Cubaris (syn.)............................ 50

schultzei, Venezillo ...................................... 50

schwabei, Styloniscus ................................ 10

schwarzi, Spherarmadillo ........................ 42

schwencki, Porcellionides ........................ 35

Scleropactes ............................................... 40

Scleropactidae................................................... 37

Scyphacella ................................................. 12

Scyphacidae ............................................. 11

secundus, Notoniscus................................. 9

sellowi, Balloniscus .................................... 31

sellowi, Philoscia (syn.) ............................. 31

senex, Sphaeroniscus ................................. 41

seriepunctata, Philoscia ........................... 22

setosus, Phalloniscus ................................ 26

seurati, Cylindroniscus ............................... 6

sexfasciatus, Porcellionides ..................... 35

silvarum, Armadillo (syn.)........................ 50

silvarum, Cubaris (syn.).......................... 50

silvarum, Venezillo ...................................... 50

silvatica, Prosekia ...................................... 22

silvaticus, Andenoniscus ........................... 12

silvestrii, Troglophiloscia ......................... 23

silvestrii, Puteoscia..................................... 23

similis, Armadillo (syn.) ............................ 50

similis, Cubaris (syn.)............................... 50

similis, Venezillo ........................................ 50

simoni, Ligia ............................................... 3

simoni, Trichorhina ................................. 30

simplex, Styloniscus .................................. 10

simrothi, Patagoniscus (syn.)................... 10

simrothi, Styloniscus ................................... 10

singularis, Archaeosia ............................... 13

singularis, Novamundoniscus................... 25

singularis, Phalloniscus (syn.) .................. 25

soyatlanensis, Armadillo (syn.) ................. 50

soyatlanensis, Venezillo.............................. 50

species, Alloniscus .................................... 11

species, Chaetophiloscia ................... 15, 22

species, Colomboscia ................................ 39

species, Ethelum ....................................... 42

species, Periscyphis .................................... 42

species, Prosekia ...................................... 22

species, Trichoniscus ................................... 8

species, Troglophiloscia ............................ 23

Sphaerillo .................................................... 45

Sphaerobathytropa ................................... 41 
sphaerocephalus, Rhyscotus.....

Sphaeroniscus ....

Spherarmadillo

spinicornis occidentalis, Porcellio (syn.).. 33

spinicornis, Porcellio ................................ 34

spinosa, Philoscia .................................... 22

spinosus, Calycuoniscus ........................... 24

spinosus, Circoniscus .............................. 38

spinosus, Paracubaris (syn.) .................... 38

spinosus, Synamardillo (syn.).................... 38

spimulosus, Tylos ............................................ 4

squamaploetelsona, Trichorhina ................ 30

squamata, Niamba ..................................... 28

squamata, Trichorhina .............................. 30

squamatus, Leptotrichus (syn.)................. 28

squamatus, Mexicostylus (syn.) ................. 30

squamatus, Microspaeroniscus ................ 19

stebbingi, Cordioniscus .............................. 9

stebbingi, Trichoniscus (syn.)..................... 9

stenocarpa, Ischioscia ........................... 17

Stenoniscidae ............................................ 10

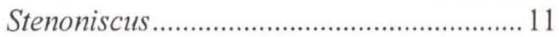

steptus, Armadilloniscus ............................ 12

sturmi, Ischioscia ....................................... 17

sturmi, Proischioscia (syn.)...................... 17

Styloniscidae ................................................. 8

Styloniscus.................................................. 9

Stymphalus ....................................................... 3

sulcata, Benthana..................................... 14

Suleoscia ....................................................... 23

sylvicola, Armadillo (syn.)......................... 51

sylvicola, Venezillo ...................................... 51

Synarmadillo ................................................... 45

Synuropus (syn.) ........................................... 40

tabularis, Psendodiploexochus .................. 45

taeniata, Benthana .................................... 14

talamancensis, Scleropactes ...................... 40

tanneri, Cubaris (syn.).............................. 51

tanneri, Venezillo ........................................... 51

tarumae, Prosekia .......................................22

tatei, Scleropactes ....................................... 40

tenuipunctatus, Bethalus ............................ 43

tertiarius, Protosphaeroniscus ................... 39

tertius, Notoniscus ..................................... 9

texensis, Rhyscotus..................................... 32

thalassophilus, Alloniscus.......................... 11

thermophila, Trichorhina ........................... 30

thlamayensis, Mexiconiscus (syn.) .............. 7

Thomasoniscus ........................................... 23

tomentosa, Trichorhina.............................. 30 tomentosus, Alloniscus (syn.) .................... 30

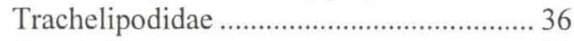

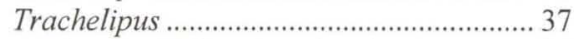

tracheofer, Ballonsicus (syn.).................... 31

Trichoniscidae ................................................. 4

trichoniscoides, Microphiloscia ............... 19

Trichoniscoides .............................................. 8

Trichoniscus ............................................... 8

Trichorhina ................................................ 28

tristani, Coxopodias (syn.) ......................... 46

tristani, Scleropactes ................................ 40

tristani, Synarmadillo ................................. 46

Troglophiloscia.......................................... 23

tropicalis, Andenoniscus........................... 12

tropicalis, Erophiloscia (syn.).................. 12

tropicalis, Littorophiloscia ........................ 18

Tropiscia .................................................. 23

truncorum, Armadillo (syn.)..................... 51

truncorum, Cubaris (syn.) ........................ 51

truncorum, Venezillo ............................... 51

tuberculatus, Armadilloniscus (syn.)......... 11

tubercululatus, Pseudarmadillo ................. 52

tuberosa, Cubaris (syn.) ........................... 51

tuberosus, Armadillo (syn.) ....................... 51

tuberosus, Venezillo................................... 51

tukeitanus, Sphaeroniscus ......................... 41

turgifrons, Rhyscotus ............................. 33

Tylidae ......................................................... 4

Tylos .............................................................. 4

Typhlotricholigoides ................................... 8

vallesensis, Cylindroniscus........................ 6

vandeli, Kuscheloniscus ............................ 9

vandeli, Novamundoniscus ....................... 25

vandeli, Phalloniscus (syn.) ...................... 25

vandeli, Trichorhina ................................. 30

Vandeloscia (syn.) ..................................... 18

vargasae, Pentoniscus .............................. 20

variegata, Ischioscia................................ 17

variegata, Ischioscia (partim syn. elongata).

vedadoensis, Leptotrichus (syn.) ............... 27

vedadoensis, Neotroponiscus ................... 27

Venezillo ........................................................ 46

venezuelae, Venezillo (syn.) ...................... 45

venezuelana, Neosanfilippia..................... 39

venusta, Cubaris (syn.)............................ 51

venustus, Armadillo (syn.) ........................ 51

venustus, Venezillo....................................... 51

veracrucensis, Trichoniscus (syn.).............. 7

veracruzana, Philoscia ................................ 22 
verrucosa, Cubaris (syn.) .......................... 51

verrucosus, Armadillo (syn.) .......................51

verrucosus, Brasiloniscus .......................... 42

verrucosus, Venezillo .................................. 51

vespertillo, Hoctunus ................................... 16

villalobosi, Brackenridgia ............................ 6

villalobosi, Protrichoniscus (syn.)................ 6

villosa, Benthana ...................................... 14

vincentis, Armadillo (syn.).........................51

vincentis, Cubaris (syn.) ............................ 51

vincentis, Venezillo .....................................51

violaceus, Microspaeroniscus.................... 39

virgatus, Porcellio (syn.) ........................... 36

virgatus, Porcellionides ................................ 36

viticola, Armadillo (syn.)............................. 51

viticola, Cubaris (syn.) ...............................51

viticola, Venezillo........................................ 51

vittata, Littorophiloscia ........................... 18

vittata, Philoscia (syn.)............................. 18

vittata, Sayoscia (syn.)........................... 18

vulgare, Armadillidium .............................. 43

walkeri, Chaetophiloscia ........................... 15

walkeri, Philoscia (syn.) ........................... 15

walkeri, Cubaris (syn.) .............................. 51

walkeri, Venezillo ...................................... 51

wartoni, Cubaris (syn.)............................ 51

wartoni, Venezillo ..................................... 51

wegeneri, Tylos ........................................... 4

welchi, Pseudarmadillo (syn.) .................... 52

werneri, Benthana ..................................... 15

wheeleri, Cubaris (syn.) ............................ 52

wheeleri, Venezillo ....................................... 52

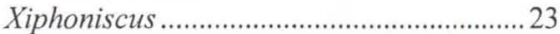

xoltumae, Trichorhina ................................ 30

yucatanensis, Antroniscus (syn.) .................. 6

yucatanensis, Cylindroniscus ...................... 6

yucatanensis, Trichorhina (syn.) ............... 30

zeteki, Scleropactes .................................... 41

zigzag, Armadillo (syn.)............................ 52

zigzag, Cubaris (syn.) ............................... 52

zigzag, Venezillo ...................................... 52

zimpanensis, Trichorhina ........................ 30

zoiai, Neosanfilippia .................................... 39 